\title{
Neural correlates of individual odor preference in Drosophila
}

\author{
Matthew A. Churgin 1 ,* \& Danylo Lavrentovich 1 ,*, Matthew A. Smith ${ }^{1,2}$, \\ Ruixuan Gao ${ }^{3,4,5}$, Edward Boyden ${ }^{3,6,7}$, Benjamin de Bivort ${ }^{1, \dagger}$ \\ 1 - Organismic and Evolutionary Biology \& Center for Brain Science, Harvard University, Cambridge, MA, USA \\ 2 - Current affiliation: Department of Entomology, University of Wisconsin-Madison, Madison WI, USA \\ 3 - McGovern Institute for Brain Research \& MIT Media Lab, MIT, Cambridge, MA, USA \\ 4 - Janelia Research Campus, Howard Hughes Medical Institute, Ashburn, VA, USA \\ 5 - Current affiliation: Biological Sciences/Chemistry, University of Illinois Chicago, Chicago, IL, USA \\ 6 - Department of Biological Engineering, MIT Center for Neurobiological Engineering, Department of Brain and Cognitive \\ Sciences \& Koch Institute, MIT, Cambridge, MA, USA \\ 7 - Howard Hughes Medical Institute, Chevy Chase, MD, USA \\ * - These authors contributed equally. \\ $\dagger$ - correspondence: debivort@oeb.harvard.edu
}

\begin{abstract}
Behavior varies even among genetically identical animals raised in the same environment ${ }^{1-10}$. However, little is known about the circuit or anatomical underpinnings of this individuality11,12. Drosophila olfaction is an ideal system for discovering the origins of behavioral individuality among genetically identical individuals. The fly olfactory circuit is well-characterized ${ }^{13-16,17-19}$ and stereotyped ${ }^{15}$, yet stable idiosyncrasies in odor preference ${ }^{4}$, neural coding 4 , and neural wiring ${ }^{20,21}$ are present and may be relevant to behavior. Using paired behavior and two-photon imaging measurements, we show that individual odor preferences in odor-vs-air and odor-vs-odor assays are predicted by idiosyncratic calcium dynamics in Olfactory Receptor Neurons (ORNs) and Projection Neurons (PNs), respectively. This suggests that circuit variation at the sensory periphery determines individual odor preferences. Furthermore, paired behavior and immunohistochemistry measurements reveal that variation in ORN presynaptic density also predicts odor-vs-odor preference. This point in the olfactory circuit appears to be a locus of individuality where microscale variation gives rise to idiosyncratic behavior. To unify these results, we constructed a leaky-integrate-andfire model of 3,062 neurons in the antennal lobe. In these simulations, stochastic fluctuations at the glomerular level, like those observed in our ORN immunohistochemistry, produce variation in PN calcium responses with the same structure as we observed experimentally, the very structure that predicts idiosyncratic behavior. Thus, our results demonstrate how minute physiological and structural variations in a neural circuit may produce individual behavior, even when genetics and environment are held constant.
\end{abstract}

Keywords: individuality, neural circuits, sensory processing, olfaction, behavioral preference, variation, antennal lobe, simulated connectome, calcium imaging, expansion imaging

\section{Main Text}

Individuals respond differently to identical sensory stimulation. We hypothesize that as sensory cues are encoded and transformed to produce motor outputs, their representation in the nervous system will become increasingly idiosyncratic and predictive of individual behavioral responses. We refer to the sites at which this idiosyncrasy emerges as "loci of individuality." To find these loci in the olfactory circuit (Fig 1a), we measured odor preferences and neural responses 4 in the same individuals, and asked whether the latter predicted the former (Fig 1b-d). Focusing on behavioral variation within a genotype, we used isogenic animals expressing the fluorescent calcium reporter GCamp6m ${ }^{22}$ in either of the two most 
bioRxiv preprint doi: https://doi.org/10.1101/2021.12.24.474127; this version posted December 24, 2021. The copyright holder for this preprint (which was not certified by peer review) is the author/funder, who has granted bioRxiv a license to display the preprint in perpetuity. It is made available under aCC-BY 4.0 International license.

Churgin \& Lavrentovich et al., 2021 - preprint version - www.biorxiv.org
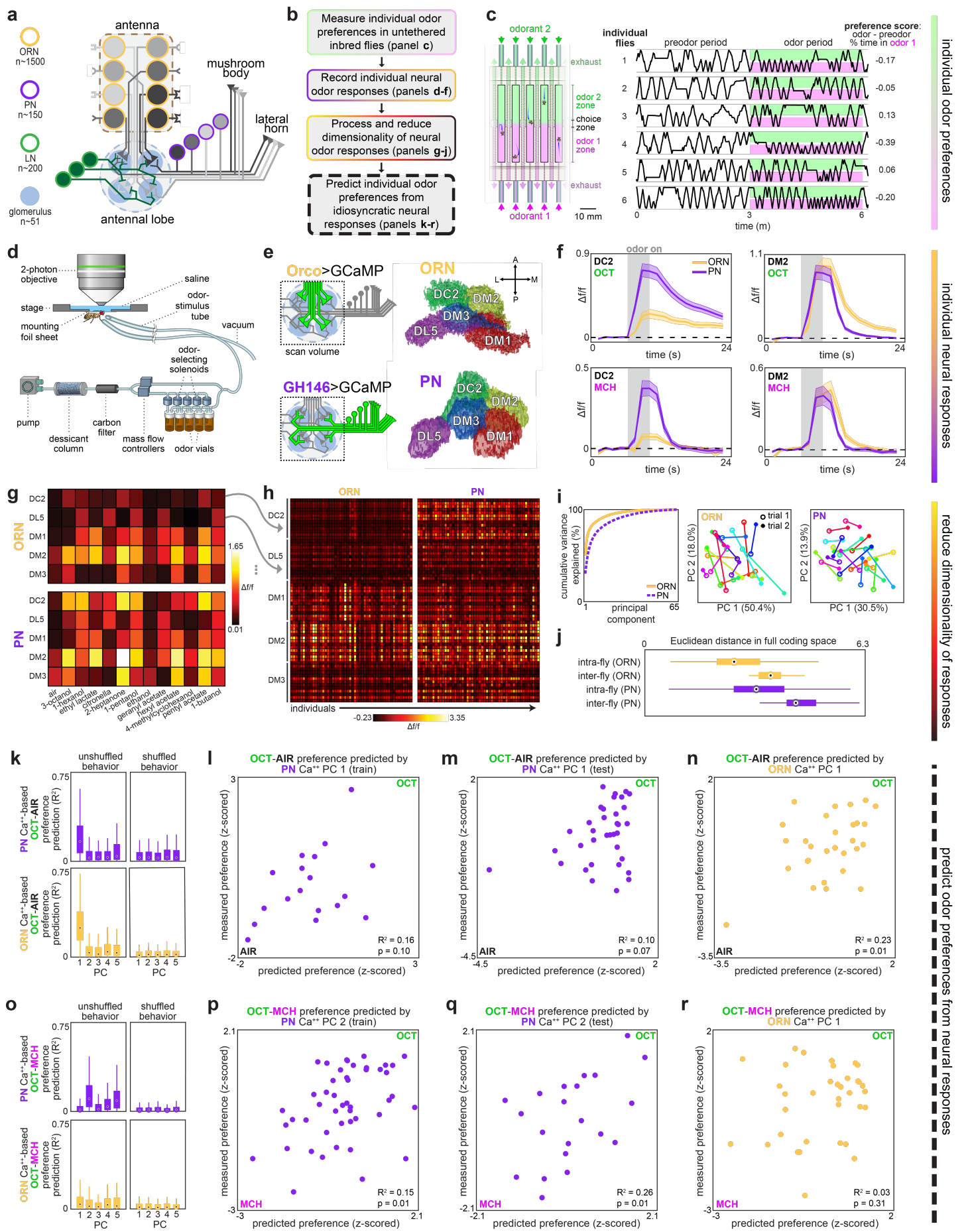

Figure 1: Idiosyncratic calcium dynamics predict individual odor preferences (a) Olfactory circuit schematic. Olfactory receptor neurons (ORNs, peach outline) and projection neurons (PNs, plum outline) are comprised of $\sim 51$ classes corresponding to odor receptor response channels. ORNs of the same class (gray shading) sense odors in the antennae and synapse together on dendrites of PNs of the same class in ball-shaped structures called glomeruli located in the antennal lobe (AL). Local neurons (LNs, green outline) mediate interglomerular cross-talk and presynaptic inhibition, amongst other roles ${ }^{73,74}$. Odor signals are normalized and whitened in the AL before being sent to the mushroom body and lateral horn for further processing. Schematic adapted from ${ }^{4}$. (b) Experiment outline. (c) Odor preference behavior tracking setup (reproduced from ${ }^{4}$ ) and example individual fly ethograms. OCT (green backdrop) and MCH (magenta backdrop) were presented for 3 minutes. (d) Head-fixed 2-photon calcium imaging and odor delivery setup (reproduced from ${ }^{4}$ ). (e) Orco and GH146 driver expression profiles (left) and example segmentation masks (right) extracted from 2-photon calcium images for a single fly expressing Orco $>$ GCaMP6m (top, expressed in a subset of all ORN classes) or GH146>Gcamp6m (bottom, expressed in a subset of all PN classes). (f) Time-dependent $\Delta \mathrm{f} / \mathrm{f}$ for glomerular odor responses in ORNs (peach) and PNs (plum) averaged across all individuals: DC2 to OCT (upper left), DM2 to OCT (upper right), DC2 to MCH (lower left), and DM2 to OCT (lower right). Shaded error bars represent S.E.M. (g) Peak $\Delta \mathrm{f} / \mathrm{f}$ for each glomerulus-odor pair averaged across all flies. (h) Individual neural responses measured in ORNs (left) or PNs (right) for 50 flies each. Columns represent the average of up to 4 odor responses from a single fly. Each row represents one glomerulus-odor response pair. Odors are the same as in panel (g). (i) Principal component analysis of individual neural ... 
Churgin \& Lavrentovich et al., 2021 - preprint version - www.biorxiv.org

Figure 1 caption (continued): ... responses. Fraction of variance explained versus principal component number (left). Trial 1 and trial 2 of ORN (middle) and PN (right) responses for 20 individuals (unique colors) embedded in PC 1-2 space. Note the variance explained by each PC is different in the case of ORN and PN responses. (j) Euclidean distances between glomerulus-odor responses within and across flies measured in ORNs ( $\mathrm{n}=65$ flies) and PNs ( $\mathrm{n}=122$ flies). Distances calculated without PCA compression. Points represent the median value, boxes represent the interquartile range, and whiskers the range of the data. (k) Bootstrapped $\mathrm{R}^{2}$ of OCT-AIR preference prediction from each of the first 5 principal components of neural activity measured in PNs (top, training set) or ORNs (bottom, all data). (l) Measured OCT-AIR preference versus preference predicted from PC 1 of PN activity in a training set ( $\mathrm{n}=18$ flies). (m) Measured OCT-AIR preference versus preference predicted from PC 1 on PN activity in a test set ( $\mathrm{n}=35$ flies) evaluated on a model trained on data from panel (1). (n) Measured OCT-AIR preference versus preference predicted from PC 1 of ORN activity ( $\mathrm{n}=30$ flies). (o) Bootstrapped $\mathrm{R}^{2}$ of OCT-MCH preference prediction from each of the first 5 principal components of neural activity measured in PNs (top, training set) or ORNs (bottom, all data). (p) Measured OCT-MCH preference versus preference predicted from PC 2 of PN activity in a training set ( $\mathrm{n}=47$ flies). (q) Measured OCT-MCH preference versus preference predicted from PC 2 on PN activity in a test set ( $\mathrm{n}=22$ flies) evaluated on a model trained on data from panel (p). (r) Measured OCT-MCH preference versus preference predicted from PC 1 of ORN activity ( $\mathrm{n}=35$ flies).

peripheral neural subpopulations of the Drosophila olfactory circuit, ORNs or PNs (Fig 1e). We performed head-fixed 2-photon calcium imaging after measuring odor preference in an untethered assay ${ }^{4}$ (Fig 1b-d, Fig S1a). Importantly, individual odor preferences are stable over timescales longer than this experiment (Fig S1b). Individual identity was maintained between behavior and imaging experiments by housing flies singly in 96well culture plates ${ }^{23}$.

We measured volumetric calcium responses in the antennal lobe (AL), the site at which ORNs synapse onto PNs in $\sim 50$ discrete microcircuits called glomeruli (Fig 1a) ${ }^{19,24}$. Flies were stimulated with a panel of 12 odors plus air (Fig 1d, Figs S2,3) and we used $k$-means clustering to automatically segment the voxels of 5 glomeruli from the resulting 4-D calcium image stacks (Fig 1e, Fig S4, Methods) ${ }^{19}$. We found that both ORN and PN odor responses were roughly stereotyped across individuals (Fig 1g,h), but also idiosyncratic as expected ${ }^{4}$. Responses in PNs appeared to be more idiosyncratic than ORNs (Fig 1j). We quantified this by training a logistic linear classifier to decode fly identity from glomerular responses. The classifier always attained a higher accuracy when trained on PN than ORN responses (Supplementary Figure 5a). While the responses of single ORNs are known to vary more than those of single $\mathrm{PNs}^{17}$, our recordings represent the total response of all ORNs or PNs in a glomerulus. This might explain our observation that ORNs exhibited less idiosyncrasy than PNs. We also found that PN responses were more variable within flies, as measured across the left and right hemisphere ALs, compared to ORN responses (Fig S5c). Higher idiosyncrasy in PNs is consistent with the hypothesis that odor representations become more idiosyncratic farther from the sensory periphery.

Next we analyzed the relationship of idiosyncratic coding to odor preference, by asking in which neurons (if any) did calcium responses predict individual preferences of flies choosing between air and an aversive odor (3-octanol, OCT; Fig S1b,c; Movie 1). Because we could potentially predict preference (a single value) using numerous glomerular-odor predictors, and had a limited number of observations (dozens), we used dimensional reduction to make parsimonious predictions. We computed the principal components (PCs) of the glomerulus-odor responses (in either ORNs or PNs) across individuals (Fig 1g-i, Fig S5-7). Using each of the first 5 PCs of PN calcium responses to linearly predict individual behavior, we found that PC 1 explained $16 \%$ of preference variance in a training set of 18 flies (Fig 1k,l). This PC 1-based model explained $10 \%$ of preference variance when evaluated on data from test flies $(n=35)$ acquired after fitting the model (Fig 1m). Moreover, we found that PC 1 of ORN neural activity explained $23 \%$ of preference variance (Fig $1 \mathrm{k}, \mathrm{n}$ ). Our interpretation is that ORN responses are idiosyncratic and predict individual odor-vs-air preference, and that these idiosyncrasies are transmitted to PNs, where they remain predictive of behavioral responses.

How should we interpret PC1 of ORN responses predicting 23\% of the variance of odor preference? This value will fall short of $100 \%$ through a combination of two factors: 1) ORN responses not having a linear relationship to behavior, and 2) noise in the measurement of ORN responses and behavior fundamentally capping the prediction performance. To disentangle these effects, and estimate the extent to which the model captured meaningful biological signal, we performed a statistical analysis (Fig S8) that factored out estimated error in measuring behavior (Fig S1be) from the model performance. This analysis implies that our model predicting odor-vs-air behavior from PC1 of ORN calcium responses (nominal $\mathrm{R}^{2}=0.23$ ) explains $\sim 80 \%$ of the biological signal between these measures. This makes intuitive sense because the raw model $\mathrm{R}^{2}(0.23)$ is close to the behavior repeatability $\mathrm{R}^{2}(0.27)$, an upper-limit (Fig S1b). In contrast, the model predicting odor-vs-air behavior from PC1 of PN calcium responses explains $\sim 50 \%$ of the biological signal (Fig S9a).

Variation in the sensory periphery has been previously implicated as a driver of behavioral variation 25,26 , but we wondered whether ORNs would be a locus of individuality for a behavior requiring the comparison of two odors (rather than just the sensation of a single odor). So we next determined if idiosyncratic calcium responses (in ORNs or PNs) could predict individual preferences in an odor-vs-odor choice (Fig S1d,e; 4; Movie 2), specifically between one aversive monomolecular odorant (OCT) and another (4-methylcyclohexanol, MCH). As before, we assessed if any of the first 5 PCs of PN calcium responses was a linear predictor of individual odor-vs-odor preferences. In this case, we found that PC 2 predicted $15 \%$ of preference variance in a training set of 47 flies (Fig 1o-p). This PC 2-based model explained $26 \%$ of preference variance when evaluated on test measurements acquired after fitting the model ( $\mathrm{n}=22$ flies) (Fig 1q). We infer that this performance corresponds to explaining $\sim 80 \%$ of the biological signal between PN calcium responses and odor-vs-odor behavior (Fig S9b).

In contrast, we found that no PCs of ORN neural activity could linearly predict odor preference beyond the level of shuffled controls ( $\mathrm{n}=35$ flies) (Fig 1o,r; Fig S9b). The best ORN PC model only predicted odor-vs-odor behavior with a nominal $\mathrm{R}^{2}$ of 0.03 ( $\sim 5 \%$ of biological signal). As another check that ORN responses were not predictive of odor-vs-odor behavior, we projected the ORN data onto PC 2 of PN responses (the successful 
Churgin \& Lavrentovich et al., 2021 - preprint version - www.biorxiv.org

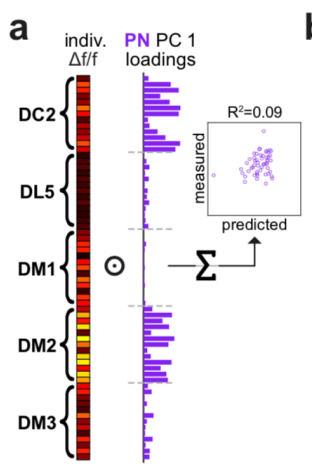

b
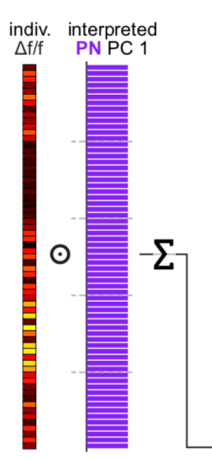

C

OCT-AIR preference predicted as "total response of all PN glomeruli" (interpretation of PN PC 1)

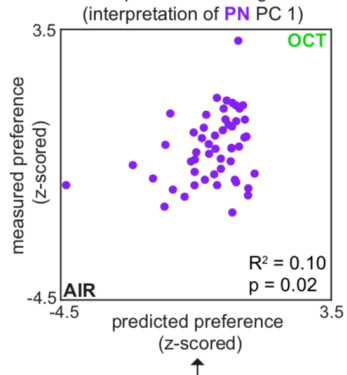

$\uparrow$
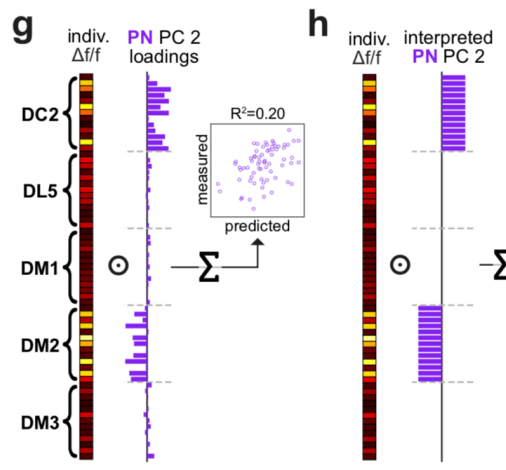

i OCT-MCH preference predicted as "DC2 response minus DM2 response" (interpretation of PN PC 2)

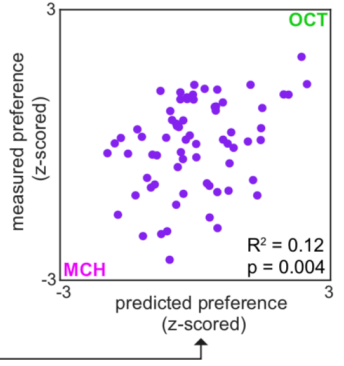

d

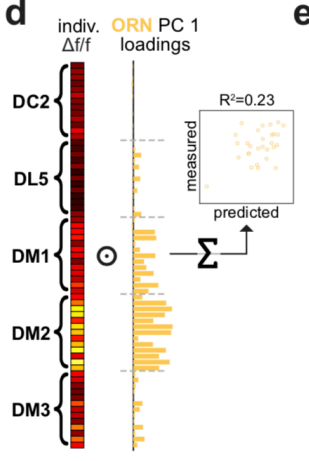

e

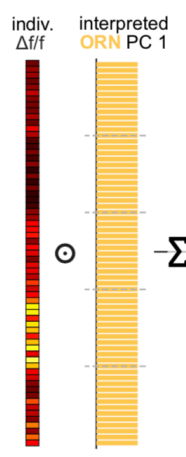

j

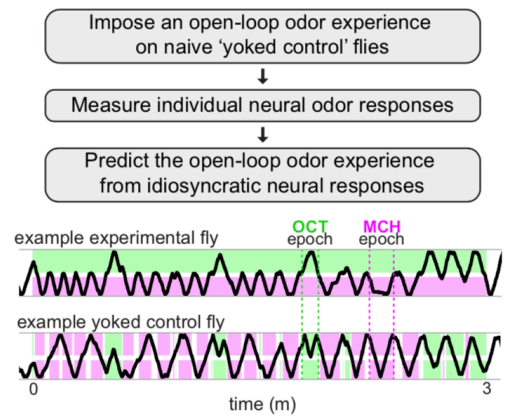

f OCT-AIR preference predicted as "total response of all ORN glomeruli" (interpretation of ORN PC 1)

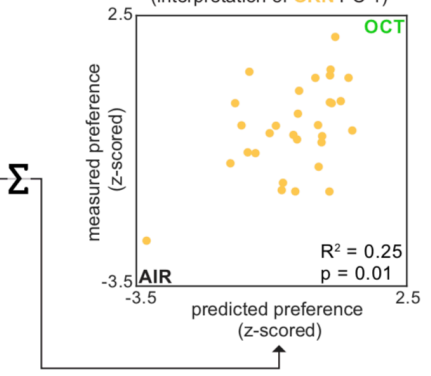

k

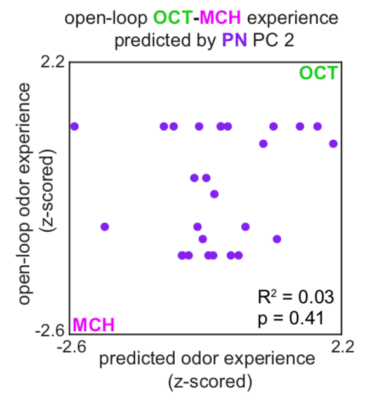

Figure 2: Variation in global and relative glomerular responses explains individual preferences (a) PC 1 loadings of PN activity for flies tested for OCT-AIR preference ( $\mathrm{n}=53$ flies). (b) Interpreted PN PC 1 loadings. (c) Measured OCT-AIR preference versus preference predicted by the average peak response across all PN coding dimensions ( $\mathrm{n}=53$ flies). (d) PC 1 loadings of ORN activity for flies tested for OCT-AIR preference $(\mathrm{n}=30$ flies). (e) Interpreted ORN PC 1 loadings. (f) Measured OCT-AIR preference versus preference predicted by the average peak response across all ORN coding dimensions ( $\mathrm{n}=30$ flies). (g) PC 2 loadings of PN activity for flies tested for OCT-MCH preference ( $\mathrm{n}=69 \mathrm{flies}$ ). (h) Interpreted PN PC 2 loadings. (i) Measured OCT-MCH preference versus preference predicted by the average peak PN response in DM2 minus DC2 across all odors ( $\mathrm{n}=69$ flies). (j) Yoked control experiment outline and example behavior traces. Experimental flies are free to move about tunnels permeated with steady state OCT and MCH flowing into either end. Yoked control flies are delivered the same odor at both ends of the tunnel which matches the odor experienced at the nose of the experimental fly at each moment in time. (k) Imposed odor experience versus the odor experience predicted from PC 2 of PN activity ( $\mathrm{n}=27$ flies) evaluated on the model trained from data in Figure 1p.

model). As expected, this did not predict odor-vs-odor behavior $\left(\mathrm{R}^{2}=0.06\right)$. Therefore, whereas idiosyncratic ORN responses (and PN responses) were predictive of odor-vs-air preferences, only PN responses were predictive of odor-vs-odor preferences.

We next sought an intuitive understanding of the models linking calcium responses and odor preference. We examined the loadings of the ORN and PN PCs and observed that variation across individuals was correlated at the level of glomeruli much more strongly than odorant (Figs S6,7). This suggests that stochastic variation in the olfactory circuit results in individual-level fluctuations in the responses of glomeruli rather than odor-specific responses. Thus, we proceeded to interpret the models in terms of how they were tuned to glomerulus-level responses. In the case of the odor-vs-air model, we noted that the loadings of PC 1 of both ORN and PN neural activity were non-negative across all glomerulus-odor response dimensions (Fig 2a, d). This suggests PC 1 captures each individual's total global response to all glomerulus-odorant combinations. To test this intuition, we assessed the performance of a linear model that simply sums the total response across all calcium dimensions (Fig 2b, e). When applied to ORN responses, this model predicted behavior with $\mathrm{R}^{2}=0.25$ ( $\sim 80 \%$ of the biological signal); in the case of PN responses, this model was somewhat predictive $\left(\mathrm{R}^{2}=0.10\right)$. For both ORNs and PNs, the model's slope parameter $(\beta)$ was negative (Table 1), meaning that stronger AL responses correlated with stronger preference for air, consistent with OCT being aversive. I.e., flies whose ORNs and PNs respond, as a population, more strongly to OCT are more likely to avoid it.

Taking this approach with the model of odor-vs-odor preference, we observed that the loadings of PC2 of PN calcium responses contrast the responses of the DM2 and DC2 glomeruli with opposing weights (Fig $2 \mathrm{~g}$ ), suggesting that the activation of DM2 relative to DC2 could predict the likelihood of a fly preferring OCT to MCH. Indeed, a linear model constructed from the total PN response in DM2 minus the total PN response in DC2 (Fig $2 \mathrm{~h}$ ) could predict individual preference for OCT versus $\mathrm{MCH}$ $\left(\mathrm{R}^{2}=0.12\right.$; explaining $\sim 40 \%$ of biological signal; Fig $\left.2 \mathrm{i}\right)$. The model beta coefficient was negative (Table 1), indicating that greater activation of DM2 vs DC2 correlates with preference for $\mathrm{MCH}$ specifically. With respect to odor-vs-odor behavior, we conclude that the relative responses of DM2 vs DC2 in PNs determines an individual's preference.

Odor experience has been shown to modulate subsequent $\mathrm{AL}$ responses ${ }^{27-29}$. This raises the possibility that our models were actually predicting individual flies' past odor experiences (i.e., the specific pattern of odor stimulation flies received in the behavioral assay) rather than their preferences. To address this possibility, we imposed the specific odor experiences of previously monitored untethered flies (in the odor-vs-odor assay) on naive 
bioRxiv preprint doi: https://doi.org/10.1101/2021.12.24.474127; this version posted December 24,2021 . The copyright holder for this preprint (which was not certified by peer review) is the author/funder, who has granted bioRxiv a license to display the preprint in perpetuity. It is made available under aCC-BY 4.0 International license.

Churgin \& Lavrentovich et al., 2021 - preprint version - www.biorxiv.org

"yoked" control flies (Fig 2j). We then measured odor responses in the PNs of these yoked flies, as before. Applying the PN PC 2 model to the yoked calcium responses did not predict flies' odor experience $\left(\mathrm{R}^{2}=0.03\right.$; Fig $\left.2 \mathrm{k}\right)$. Thus, the responses of $\mathrm{DM} 2 \mathrm{vs}$ DC2 in PNs do not predict individual open-loop odor experiences.

Previous work found that PN response transients, rather than fixed points, contain more odor identity information ${ }^{30}$. We therefore asked at which times during odor presentation an individual's neural responses could best predict odor preference. Applying each of our three successful calcium-to-behavior models (ORN PC1-odor-vs-air, PN PC1-odor-vs-air, PN PC2-odor-vsodor) to the time-varying calcium signals, we found that in all cases that behavior prediction generally rose during odor delivery (Fig S10a-c). In ORNs, the predictive accuracy remained high after odor offset, whereas in PNs it declined. Thus, the overall sensitivity of ORNs that appears to predict odor-vs-air preferences may persist after odor stimulation ends. The times during which calcium responses predicted individual behavior generally aligned to the times during which a linear classifier could decode odor identity from ORN or PN responses (Fig S10d), suggesting that idiosyncrasies in odor encoding predict individual preferences.

Pivoting to another level of biological regulation, we next investigated how structural variation in the nervous system might underlie the variations in neural activity that correlate with idiosyncratic behavior. Because PN, but not ORN, calcium responses predicted odor-vs-odor preference, we hypothesized that a circuit element between ORNs to PNs could confer onto PNs behaviorally-relevant physiological idiosyncrasies that are absent in ORNs. The ORN-PN synapse is an obvious candidate. We therefore visualized presynaptic T-bar density in ORNs using transgenic mStrawberry-tagged Brp-Short, immunohistochemistry and confocal microscopy ${ }^{31}$ after measuring individual preference for OCT versus MCH (Fig 3a, Fig S11).

We used a semi-automated custom software to segment 4 of the 5 focus glomeruli from confocal volumes of the AL (Fig 3b; DL5 was not readily segmentable across samples, but was dispensable in all behavior-predicting models). We then quantified the volume, fluorescent intensity and "density" (intensity/volume) of Brp-Short staining within each glomerulus (Fig S11a-f). Of these measures, density was the most consistent across hemispheres, while also showing variation across individuals, so we focused on this metric in subsequent analyses.

To begin assessing the relationship between presynaptic structural variation and behavior, we calculated the principal components of Brp-Short density across individuals and found that PCs 1 and 2 were qualitatively similar to those measured in our calcium imaging experiments in that PC 1 was non-negative positive across glomeruli, reflecting global average staining intensity, and PC 2 exhibited a large magnitude loading for DC2 against negative loadings for the other glomeruli (Fig S11g). As in the PN calcium response models, PC 2 of Brp-Short density afforded the best prediction of odor-vs-odor preferences in training data (Fig 3d-f, $\mathrm{R}^{2}=0.22, \mathrm{n}=22$ flies) and for test data collected after training this model (Fig $3 \mathrm{~g}, \mathrm{R}^{2}=0.08, \mathrm{n}=31$ flies). We tested our intuitive hypothesis that PC 2 captures the differential response of DM2 vs DC2, by applying the "DM2 minus
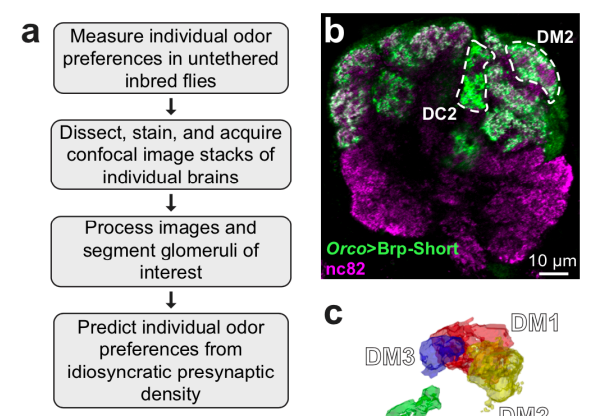

c

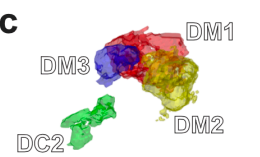

f OCT-MCH preference
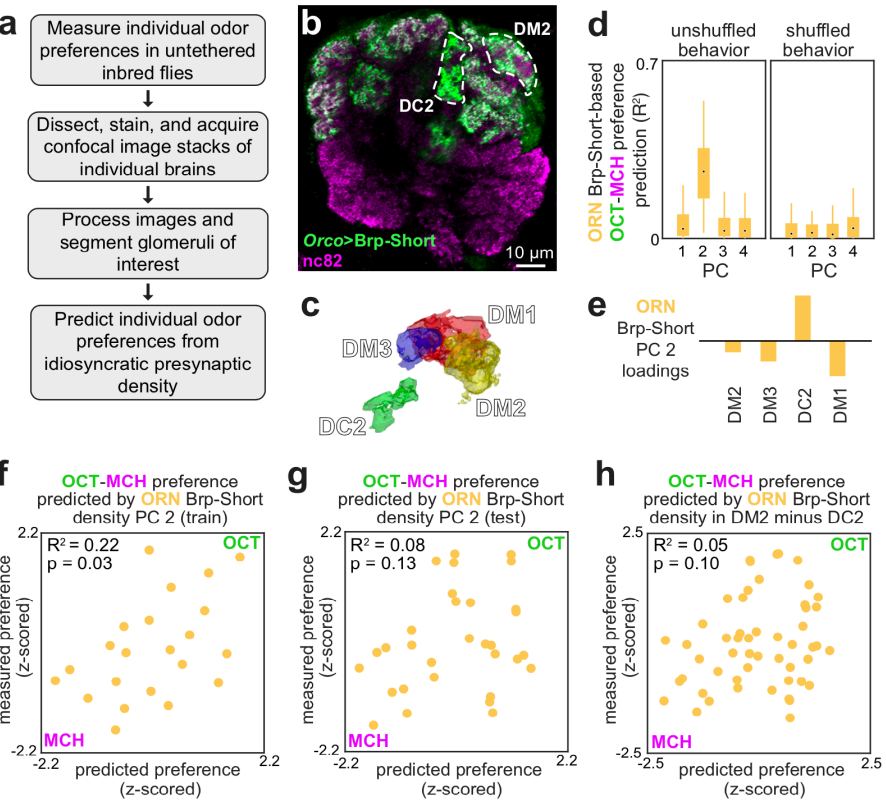

h
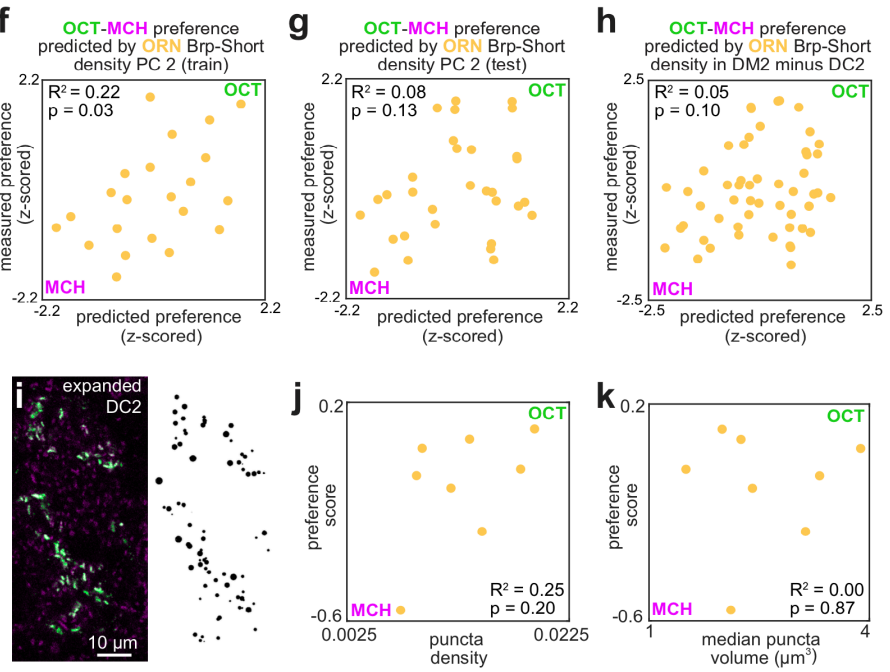

Figure 3: Idiosyncratic presynaptic marker density in DM2 and DC2 predicts OCT-MCH preference (a) Experiment outline. (b) Example slice from a $\mathrm{z}$-stack of the antennal lobe expressing Orco $>$ Brp-Short (green) with DC2 and DM2 visible (white dashed outline). nc82 counterstain (magenta). (c) Example glomerulus segmentation masks extracted from an individual z-stack. (d) Bootstrapped $\mathrm{R}^{2}$ of OCT-MCH preference prediction from each of the first 4 principal components of Brp-Short density measured in ORNs (training set, $n=22$ flies). (e) PC 2 loadings of Brp-Short density. (f) Measured OCT-MCH preference versus preference predicted from PC 2 of ORN Brp-Short density in a training set ( $\mathrm{n}=22$ flies). (g) Measured OCT-MCH preference versus preference predicted from PC 2 on ORN Brp-Short density in a test set $(n=31$ flies) evaluated on a model trained on data from panel (f). (h) Measured OCT-MCH preference versus preference predicted from ORN Brp-Short density in DM2 minus DC2 ( $n=53$ flies). (i) Example expanded AL expressing Or13a $>$ Brp-Short (left) and Imaris-identified puncta from that sample (right). (j) OCT-MCH preference score plotted against Brp-Short puncta density in expanded Or13a $>$ Brp-Short samples ( $\mathrm{n}=8$ flies). (k) OCT-MCH preference score plotted against Brp-Short median puncta volume in expanded Or13a $>$ Brp-Short samples ( $\mathrm{n}=8$ flies).

DC2 model" (Fig $2 \mathrm{~h}$ ) to the Brp-Short data. While this rudimentary model did not attain statistical significance, it trended in the right direction and had a negative beta coefficient, implying that higher presynaptic density in DM2 compared to DC2 correlates with preference for $\mathrm{MCH}$ (Table 2). This is consistent with the beta parameter of the PN calcium response model.

The range of differences between DM2 and DC2 Brp-Short staining across individuals $(-50 \%$ to $40 \%$; normalized by the average of the two glomeruli) was less than that of PN calcium response differences (-60\% to $100 \%$; Fig S12), suggesting that variation in presynaptic density is likely not the full explanation of variability in calcium responses. This is also consistent with the observation that the best presynaptic density models are less 
bioRxiv preprint doi: https://doi.org/10.1101/2021.12.24.474127; this version posted December 24,2021 . The copyright holder for this preprint (which was not certified by peer review) is the author/funder, who has granted bioRxiv a license to display the preprint in perpetuity. It is made available under aCC-BY 4.0 International license.

Churgin \& Lavrentovich et al., 2021 - preprint version - www.biorxiv.org

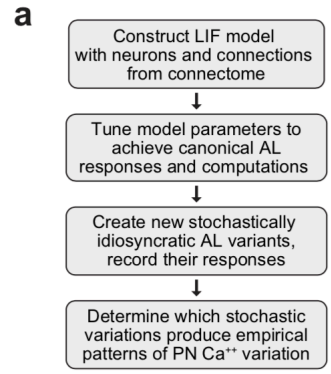

b

$$
\mathrm{C}_{i} \frac{d V_{i}}{d t}=\frac{V_{i, 0}-V_{i}(t)}{R_{i}}+I_{i, o d o r}(t)+\sum_{j=1}^{N} a_{i} W_{j i} I_{j}(t)
$$

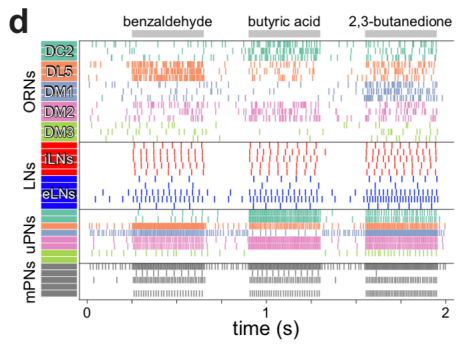

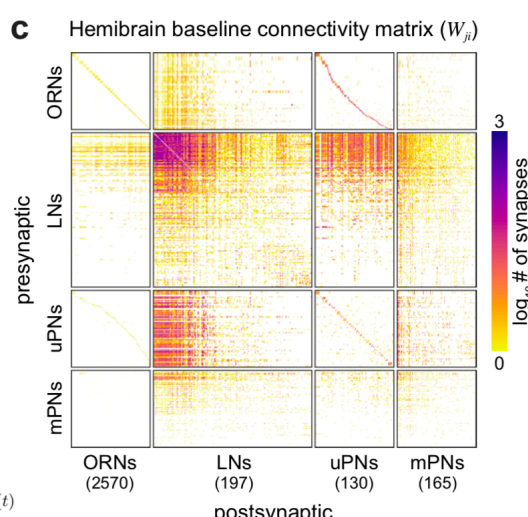

e

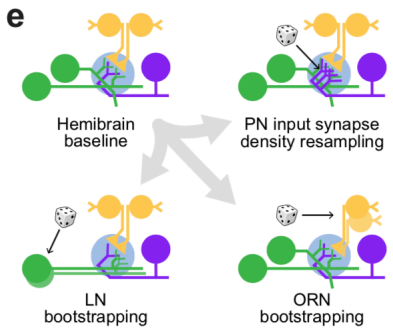

f
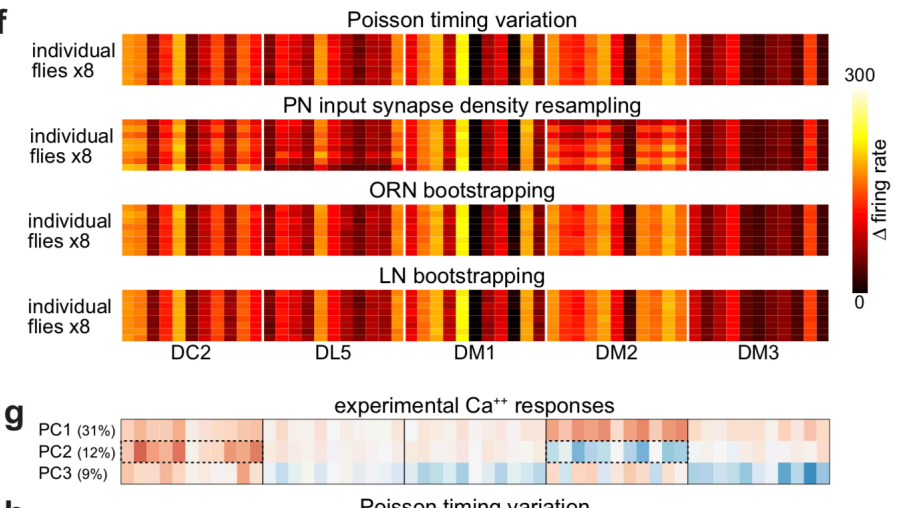

h

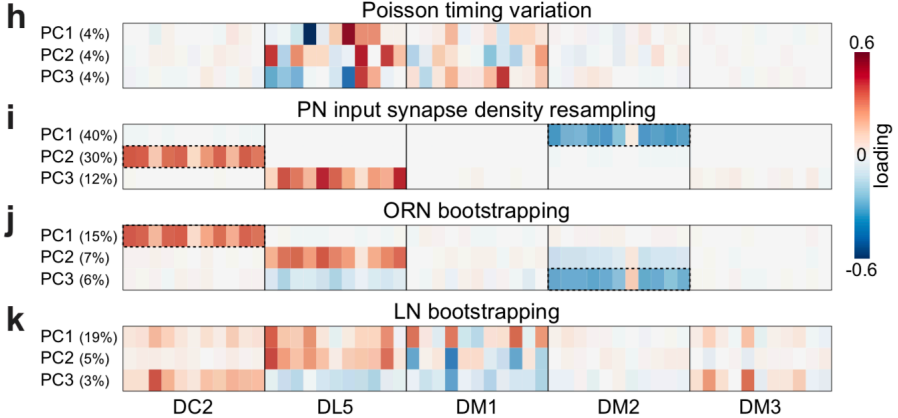

Figure 4: Simulation of developmentally stochastic olfactory circuits (a) AL modeling analysis outline. (b) Leaky-integrator dynamics of each simulated neuron. When a neuron's voltage reaches its firing threshold, a templated action potential is inserted, and downstream neurons receive a postsynaptic current. See Modeling Supplementary Methods. (c) Synaptic weight connectivity matrix, derived from the hemibrain connectome ${ }^{34}$. (d) Spike raster for randomly selected example neurons from each AL cell type. Colors indicate ORN/PN glomerular identity and LN polarity (i=inhibitory, e=excitatory). (e) Schematic illustrating sources of developmental stochasticity as implemented in the simulated AL framework. See Movies 4-6 for the effects of these resampling methods on the synaptic weight connectivity matrix. (f) PN glomerulus-odor response vectors for 8 idiosyncratic ALs subject to Input spike Poisson timing variation, PN input synapse density resampling, and ORN and LN population bootstrapping. (g) Loadings of the principal components of PN glomerulus-odor responses as observed across experimental flies (top). Dotted outlines highlight loadings selective for the DC2 and DM2 glomerular responses, which underlie predictions of individual behavioral preference. (h-k) As in (g) for simulated PN glomerulus-odor responses subject to Input spike Poisson timing variation, PN input synapse density resampling, and ORN and LN population bootstrapping. See Fig S16 for additional combinations of idiosyncrasy methods. In (f-k) the sequence of odors within each glomerular block is: OCT, 1-hexanol, ethyl-lactate, 2-heptanone, 1-pentanol, ethanol, geranyl acetate, hexyl acetate, MCH, pentyl acetate and butanol.

predictive of behavior than the the best calcium response models $\left(\mathrm{R}^{2}=.09\right.$ vs $\mathrm{R}^{2}=0.23$; capturing $\sim 30 \%$ and $\sim 80 \%$ of biological signal, respectively; Fig 1p,q vs Fig $3 \mathrm{f}, \mathrm{g}$ ). Nevertheless, these results suggest that differences in presynaptic inputs to DM2 and DC2 PNs may contribute to variation in DM2 and DC2 calcium dynamics, in turn giving rise to individual preferences for OCT versus $\mathrm{MCH}$.

To better understand what variable Brp-Short staining represented on a microstructural level, we performed paired behavior and expansion microscopy in flies expressing Brp-Short specifically in DC2-projecting ORNs (Movie 3). Using expansion microscopy 32,33 , we achieved a $\sim 4$-fold increase in linear resolution in immunostained samples, allowing us to visualize individual Brp-Short puncta (Fig 3i) ${ }^{32}$. While the sample size $(n=8)$ of this imaging pipeline did not warrant a formal modeling analysis, we observed that the trend between density of Brp-Short in DC2 and odor-vs-odor preference was consistent with a positive correlation, whereas the trend between Brp-Short volume and odor-vsodor preference was not (Fig $3 \mathrm{j}, \mathrm{k})$. These results hint that variation in the density of presynaptic sites, rather than other biophysical properties, may be a critical factor underlying physiological and behavioral individuality.

Finally, we sought an integrative understanding of how synaptic variation plays out across the olfactory circuit to produce behav- iorally-relevant physiological variation. We developed a leakyintegrate-and-fire model of the entire $\mathrm{AL}$, comprising 3,062 spiking neurons and synaptic connectivity taken directly from the Drosophila hemi brain connectome ${ }^{34}$. Our overall approach with this model (Fig 4a) was to 1) confirm that it could perform canonical AL computations, particularly the transformation of odor representations from ORNs to PNs, 2) introduce different kinds of stochastic variations to the circuit (corresponding to idiosyncratic developmental variants of the AL), and 3) determine what kind(s) of stochastic variation produce the patterns of idiosyncratic PN physiological variation we observed in our calcium imaging experiments. This strategy has the potential to link developmental variation at the synapse level to the physiological variation that appears to be driving behavioral individuality.

The biophysical properties of neurons in our model (Fig 4b, Table 3) were determined by published electrophysiological studies (See Modeling Supplementary Methods) and similar to those used in previous fly models 35,36 . The polarity of neurons was determined largely by their cell type (ORNs are excitatory, PNs predominantly excitatory, and LNs predominantly inhibitory. See Supplementary Methods for details). The strength of synaptic connections between any pair of AL neurons was given by the hemibrain connectome ${ }^{34}$ (Fig 4c). Odor inputs were simulated by injecting current into ORNs to produce firing rates in those neurons that match published ORN-odor recordings ${ }^{37}$, and the out- 
Churgin \& Lavrentovich et al., 2021 - preprint version - www.biorxiv.org

put of the system was recorded as the firing rates of PNs during odor stimulation (Fig 4d). At this point, there remained only four free parameters in our model, the relative sensitivity (postsynaptic current per upstream action potential) of each AL cell type (ORNs, PNs, excitatory LNs and inhibitory LNs). We explored this parameter space manually, and identified a configuration in which AL simulation (Fig S13) recapitulated four canonical properties seen experimentally (Fig S14): 1) typical firing rates at baseline and during odor stimulation $18,38-40,2$ ) a more uniform distribution of $\mathrm{PN}$ firing rates compared to $\mathrm{ORN}$ rates ${ }^{18}, 3$ ) greater separation of PN odor representations compared to ORN representations ${ }^{18}$, and 4) a sub-linear transfer function between ORNs and $\mathrm{PNs}^{18}$. Thus, our simulated AL appeared to perform the fundamental computations of real ALs, providing a baseline for assessing the effects of idiosyncratic variation.

We simulated stochastic individuality in the AL circuit in two ways (Fig 4e): glomerular-level variation in PN input-synapse density and bootstrapping of neuronal compositions within cell types. The former reflects a statistical relationship observed between glomerular volume and synapse density within the hemibrain connectome (Fig S15). The latter reflects variety in the outcomes of the developmental programs for ORNs, PNs, etc. Movies 4-6 show the diverse connectivity matrices attained under these resampling approaches. We simulated odor responses in thousands of ALs made idiosyncratic by these sources of variation, and in each, recorded the firing rates of PNs when stimulated by the 12 odors from our experimental panel (Fig 4f, Fig S13). We also recorded the effect of random Poisson timing of input spikes in the absence of circuit idiosyncrasy.

To determine which, if any, sources of variation produced patterns of PN coding variation consistent with our empirical measurements, we examined the loadings of the principal components of PN responses across real idiosyncratic flies and simulated idiosyncratic ALs. Per above, empirical PN responses are strongly correlated at the level of glomeruli (Figs 4g, Figs S6, $\mathrm{S} 7)$. In the model, resampling PN input-synapse density across glomeruli produced PN response correlations strongly organized by glomerulus, not surprisingly (Fig $4 \mathrm{~h}$ ). This served as a positive control that the AL model can recapitulate the empirical structure of PN response variation. As a negative control, variation in PN responses due solely to poisson timing of ORN input spikes (i.e., absent any circuit idiosyncrasy) was not organized at the glomerular level (Fig 4i). Strikingly, bootstrapping ORN membership yielded a strong glomerular organization in PN responses (Fig 4j). Bootstrapping LNs, in contrast, produced much less glomerular organization (Fig 4k). The PCA loadings for PN responses under all combinations of cell type bootstrapping and PN input-synapse density resampling are given in Fig S16.

Notably, DM2 and DC2 (also DL5) stand out in the PCA loadings under PN input-synapse density resampling and ORN bootstrapping (Fig 4i,j), suggesting that behaviorally-relevant PN coding variation is recapitulated in this modeling framework. To formalize this analysis, for each idiosyncratic AL, we computed a "behavioral preference" by applying the PN PC2 linear model in Fig 1o-q. We then determined how accurately a linear classifier could distinguish OCT- vs MCH-preferring ALs in the space of the first 3 principal components of PN responses, as a function of the source of idiosyncrasy (Fig S17). High accuracy was attained with sources of circuit variation within PN loadings highlighting DM2 and DC2 (namely PN input-synapse density resampling and ORN bootstrapping). Thus, developmental variability in ORN populations may drive patterns of PN physiological variation that in turn drive individuality in odor-vs-odor choice behavior.

\section{Discussion}

We found elements of the Drosophila olfactory circuit where patterns of physiological activity emerge that are predictive of individual behavioral preferences. These circuit elements can be considered loci of individuality, as they appear to harbor the origins of idiosyncratic preferences among isogenic animals reared in the same environment. Specifically, we found that the total responsiveness of ORNs predicts idiosyncratic preferences in an odor-vs-air assay, and that contrasting glomerular activation in PNs predicts idiosyncratic preferences in an odor-vs-odor assay (Figs 1,2). Both of these circuit elements are in the olfactory sensory periphery, suggesting that behavioral idiosyncrasy arises early in the sensorimotor transformation.

Previous work has found mammalian peripheral circuit areas are predictive of individual behavior $25,26,41,42$, but this study is among the first $11,12,43$ to link cellular-level circuit variants and individual behavior in the absence of genetic variation. Another key conclusion of our study is that loci of individuality are likely to vary, even within the sensory periphery, with the specific behavioral paradigm (i.e., odor-vs-odor or odor-vs-air). Our ability to predict behavioral preferences was limited by the repeatability of the behavior itself (Fig S8). Low persistence of odor preference may be attributable to factors like internal states or plasticity. It may be fruitful in future studies to map circuit elements whose activity predicts trial-to-trial behavioral fluctuations within individuals.

Seeking insight into the molecular basis of behaviorally-relevant physiological variation, we imaged Brp in the axon terminals of the ORN-PN synapse, using confocal and expansion microscopy. We found that Brp glomerular (and probably puncta) density was a predictor of individual odor-vs-odor preferences (Fig 3). Higher Brp in the DM2 glomerulus predicted stronger MCH preference, like higher calcium responses in DM2 PNs, suggesting that variation in PN inputs might underlie PN physiological variation. This is consistent with the recent finding of a linear relationship between synaptic density and excitatory postsynaptic potentials $^{44}$ and another study in which idiosyncratic synaptic density in central complex output neurons (measured by light microscopy) predicts individual locomotor behavior ${ }^{12}$. The predictive relationship between Brp and behavior was weaker than that of PN calcium responses, suggesting there are other determinants, such as other synaptic proteins, neurite morphology, or the influence of idiosyncratic $\mathrm{LNs}^{21}$ modulating the ORN-PN transformation ${ }^{45}$.

To integrate our synaptic and physiological results, in the context of the whole olfactory circuit, we implemented a spiking model with 3,062 neurons and synaptic weights drawn directly from the fly connectome ${ }^{34}$ (Fig 4). With light parameter tuning, this model recapitulated canonical AL computations, providing a baseline for assessing the effects of idiosyncratic stochastic variation. We simulated individual ALs with a variety of sources of variation, 
Churgin \& Lavrentovich et al., 2021 - preprint version - www.biorxiv.org

reflecting developmental stochasticity in PN input synapse density and the population composition of AL cell types. Two sources of variation (PN input synapse density and ORN bootstrapping) produced patterns of PN response variation that matched our experimental results, namely correlated responses across odors in the DC2 and DM2 glomeruli. These sources of variation specifically implicate the ORN-PN synapse (like our Brp results) as an important substrate for establishing behaviorally-relevant patterns of $\mathrm{PN}$ response variation.

The flies used in our experiments were isogenic and reared in standardized laboratory conditions that produce reduced behavioral individuality compared to enriched environments ${ }^{46-48}$. Yet, even these conditions yield substantial behavioral individuality. How this happens is unclear, with possibilities ranging from thermal fluctuations at the molecular scale to macroscopic, but seemingly irrelevant, variations like the exact fill level of the culture media ${ }^{49}$. Developing nervous systems employ various compensation mechanisms to dampen out the effects of these fluctuations $\mathrm{s}^{9,20}$. But behavioral variation may be beneficial, supporting a bet-hedging strategy 50 to counter environmental fluctuations ${ }^{4,51-53}$. Empirically, the net effect of dampening and accreted ontological ${ }^{54}$ fluctuations is individuals with diverse behaviors. This process unfolds across all levels of biological regulation. Just as PN response variation appears to be partially rooted in glomerular Brp variation, the latter has its own molecular roots, including, perhaps, stochasticity in gene expression ${ }^{55,56}$, itself a predictor of idiosyncratic behavioral biases ${ }^{57}$. Improved methods to longitudinally assay the fine-scale molecular and anatomical makeup of behaving organisms throughout development and adulthood will be invaluable to further illuminate the mechanistic origins of individuality.

\section{Methods}

\section{Data and code}

All data supporting these results and the analysis code are available at http://lab.debivort.org/odor-loci-of-individuality/.

\section{Fly rearing}

Experimental flies were reared in a Drosophila incubator (Percival Scientific DR-36VL) at $22^{\circ} \mathrm{C}, 40 \%$ relative humidity, and $12: 12 \mathrm{~h}$ light:dark cycle. Flies were fed cornmeal/dextrose medium, as previously described ${ }^{4}$. Mated female flies aged 3 days post-eclosion were used for behavioral persistence experiments. Mated female flies aged 7 to 15 days post-eclosion were used for all paired behavior-calcium imaging and immunohistochemistry experiments.

\section{Fly stocks}

The following stocks were obtained from the Bloomington Drosophila Stock Center: P\{20XUAS-IVS-GCaMP6m $\}$ attP40 (BDSC \#42748), w[*]; P $\{\mathrm{w}[+\mathrm{mC}]=$ Or13a-GAL4.F $\} 40.1$ (BDSC \#9945), w[*]; P $\{\mathrm{w}[+\mathrm{mC}]=$ Or19a-GAL4.F 61.1 (BDSC \#9947), $\mathrm{w}\left[{ }^{*}\right] ; \mathrm{P}\{\mathrm{w}[+\mathrm{mC}]=\mathrm{Or} 22 \mathrm{a}-\mathrm{GAL} 4.7 .717\} 14.2 \quad(\mathrm{BDSC} \# 9951)$, $\mathrm{w}[*] ; \quad \mathrm{P}\{\mathrm{w}[+\mathrm{mC}]=$ Orco-GAL4.W $\} 11.17 ; \mathrm{TM} 2 / \mathrm{TM} 6 \mathrm{~B}, \mathrm{~Tb}[1]$ (BDSC \#26818). Transgenic lines were outcrossed to the iso- genic line isokh $11^{4}$ for at least 5 generations prior to being used in any experiments. GH146-Gal4 was a gift provided by Y. Zhong ${ }^{4}$. w; UAS-Brp-Short-mStrawberry; UAS-mCD8-GFP; + was a gift of Timothy Mosca and was not outcrossed to the isokh11 background ${ }^{31}$.

\section{Odor delivery}

Odor delivery during behavioral tracking and neural activity imaging was controlled with isolation valve solenoids (NResearch Inc. $)^{4}$. Saturated headspace from $40 \mathrm{ml}$ vials containing 5 $\mathrm{ml}$ pure odorant were serially diluted via carbon-filtered air to generate a variably (10-25\%) saturated airstream controlled by digital flow controllers (Alicat Scientific) and presented to flies at total flow rates of $\sim 100 \mathrm{~mL} / \mathrm{min}$. The odor panel used for imaging was comprised of the following odorants: 2-heptanone (CAS \#110-43-0, Millipore Sigma), 1-pentanol (CAS \#71-41-0, Millipore Sigma), 3-octanol (CAS \#589-98-0, Millipore Sigma), hexyl-acetate (CAS \#142-92-7, Millipore Sigma), 4-methylcyclohexanol (CAS \#589-91-3, Millipore Sigma), pentyl acetate (CAS \#628-63-7, Millipore Sigma), 1-butanol (CAS \#71-36-3, Millipore Sigma), ethyl lactate (CAS \#97-64-3, Millipore Sigma), geranyl acetate (CAS \#105-87-3, Millipore Sigma), 1hexanol (CAS \#111-27-34, Millipore Sigma), citronella java essential oil (191112, Aura Cacia), and 200 proof ethanol (V1001, Decon Labs).

\section{Odor preference behavior}

Odor preference was measured at $25^{\circ} \mathrm{C}$ and $20 \%$ relative humidity. As previously described ${ }^{4}$, individual flies confined to custom-fabricated tunnels were illuminated with infrared light and behavior was recorded with a digital camera (Basler) and zoom lens (Pentax). The odor choice tunnels were $50 \mathrm{~mm}$ long, $5 \mathrm{~mm}$ wide, and $1.3 \mathrm{~mm}$ tall. Custom real-time tracking software written in Matlab was used to track centroid, velocity, and principal body axis angle throughout the behavioral experiment, as previously described ${ }^{4}$. After a 3-minute acclimation period, odorants were delivered to either end of the tunnel array for 3 minutes. Odor preference score was calculated as the fraction of time spent in the reference side of the tunnel during odor-on period minus the time spent in the reference side of the tunnel during the pre-odor acclimation period.

\section{Behavioral preference persistence measurements}

After measuring odor preference, flies were stored in individual housing fly plates (modified 96-well plates; FlySorter, LLC) on standard food, temperature, humidity, and lighting conditions. Odor preference of the same individuals was measured 3 and/or 24 hours later. In some cases, fly tunnel position was randomized between measurements. We observed that randomization had little effect on preference persistence.

\section{Calcium imaging}

Flies expressing GCaMP6m in defined neural subpopulations were imaged using a custom-built two-photon microscope and ultrafast Ti:Sapphire laser (Spectra-Physics Mai Tai) tuned to $930 \mathrm{~nm}$. For paired behavior and imaging experiments, the time elapsed between behavior measurement and imaging ranged from 15 minutes to 3 hours. Flies were anesthetized on ice and 
Churgin \& Lavrentovich et al., 2021 - preprint version - www.biorxiv.org

immobilized in an aluminum sheet with a female-fly-sized hole cut in it. The head cuticle between the antennae and ocelli was removed along with the tracheae to expose the ALs from the dorsal side. Volume scanning was performed using a piezoelectric objective mount (Physik Instrumente). ScanImage 2013 software (Vidrio Technologies) was used to coordinate galvanometer laser scanning and image acquisition. Custom Matlab (Mathworks) scripts were used to coordinate image acquisition and control odor delivery. 256 by 192 (x-y) pixel 16-bit tiff images were recorded. The piezo travel distance was adjusted between 70 and $90 \mu \mathrm{m}$ so as to cover most of the AL. The number of z-sections in a given odor panel delivery varied between 7 and 12 yielding a volume acquisition rate of $0.833 \mathrm{~Hz}$. Odor delivery occurred from 6-9.6s of each recording.

Each fly experienced up to four deliveries of the odor panel. The antennal lobe being recorded (left or right) was alternated after each successful completion of an odor panel. Odors were delivered in randomized order. In cases where baseline fluorescence was very weak or no obvious odor responses were visible, not all four panels were delivered.

\section{Glomerulus segmentation and labeling}

Glomerular segmentation masks were extracted from raw image stacks using a $k$-means clustering algorithm based on time-varying voxel fluorescence intensities, as previously described ${ }^{4}$. Each image stack, corresponding to a single odor panel delivery, was processed individually. Time-varying voxel fluorescence values for each odor delivery were concatenated to yield a voxel-bytime matrix consisting of each voxel's recorded value during the course of all 13 odor deliveries of the odor panel. After z-scoring, principal component analysis was performed on this matrix and $75 \%$ of the variance was retained. Next, $k$-means $(\mathrm{k}=80,50$ replicates with random starting seeds) was performed to produce 50 distinct voxel cluster assignment maps which we next used to calculate a consensus map. This approach was more accurate than clustering based on a single $k$-means seed.

Of the 50 generated voxel cluster assignment maps, the top 5 were selected by choosing those maps with the lowest average within-cluster sum of distances, selecting for compact glomeruli. The remaining maps were discarded. Next, all isolated voxel islands in each of the top 5 maps were identified and pruned based on size (minimum size $=100$ voxels, maximum size $=$ 10000 voxels). Finally, consensus clusters were calculated by finding voxel islands with significant overlap across all 5 of the pruned maps. Voxels which fell within a given cluster across all 5 pruned maps were added to the consensus cluster. This process was repeated for all clusters until the single consensus cluster map was complete. In some cases we found by manual inspection that some individual glomeruli were clearly split into two discrete clusters. These splits were remedied by automatically merging all consensus clusters whose centroids were separated by a physical distance of less than 30 voxels and whose peak odor response Spearman correlation was greater than 0.8. Finally, glomeruli were manually labeled based on anatomical position, morphology, and size 24 . We focused our analysis on 5 glomeruli (DM1, DM2, DM3, DL5, and DC2) which could be observed in most of our recordings. However, not all 5 glomeruli were identified in all recordings (Fig S3). Missing glomerular data was later mean-imputed.

\section{Calcium image data analysis}

All data was processed and analyzed in Matlab 2018a (The Mathworks, Natick, MA USA). Calcium responses for each voxel were calculated as $\Delta \mathrm{f} / \mathrm{f}=[\mathrm{f}(\mathrm{t})-\mathrm{F}] / \mathrm{F}$, where $\mathrm{f}(\mathrm{t})$ and $\mathrm{F}$ are the instantaneous and average fluorescence, respectively. Each glomerulus' time-dependent calcium response was calculated as the mean $\Delta \mathrm{f} / \mathrm{f}$ across all voxels falling within the glomerulus' automatically-generated segmentation mask during a single volume acquisition. Time-varying odor responses were normalized to baseline by subtracting the median of pre-odor $\Delta \mathrm{f} / \mathrm{f}$ from each trace. Peak odor response was calculated as the maximum fluorescence signal from $7.2 \mathrm{~s}$ to $10.8 \mathrm{~s}$ (images 6 through 9) of the recording.

To compute principal components of calcium dynamics, each fly's complement of odor panel responses (a 5 glomeruli by 13 odors $=65$-dimensional vector) was concatenated. Missing glomerulus-odor response values were filled in with the mean glomerulus-odor pair across all fly recordings for which the data was not missing. After infilling, principal component analysis was carried out with individual odor panel deliveries as observations and glomerulus-odor responses pairs as features.

In a subset of experiments in which we imaged calcium activity, some solenoids failed to open, resulting in the failure of odor delivery in a small number of trials. In these cases, we identified trials with valve failures by manually recognizing that glomeruli failed to respond during the nominal odor period. These trials were treated as missing data and infilled, as described above. Fewer than $\sim 10 \%$ of flies and $5 \%$ of odor trials were affected.

For all predictive models constructed, the average principal component score or glomerulus-odor $\Delta \mathrm{f} / \mathrm{f}$ response across trials was used per individual; that is, each fly contributed one data point to the relevant model. Linear models were constructed from behavior scores and the relevant predictor (principal component, average $\Delta \mathrm{f} / \mathrm{f}$ across dimensions, specific glomerulus measurements) as described in the text and Tables 1-2. To predict behavior as a function of time during odor delivery, we analyzed data as described above, but considered only $\Delta \mathrm{f} / \mathrm{f}$ at each single time point (Fig S10), rather than averaging during the peak response interval.

To decode individual identity from neural responses, we first performed PCA on individual odor panel peak responses. We retained principal component scores constituting specified fractions of variance (Extended Figure 4d) and trained a linear logistic classifier to predict individual identity from single odor panel deliveries.

To decode odor identity from neural responses, each of the 5 recorded glomeruli were used as features, and the calcium response of each glomerulus to a specific odor at a specified time point were used as observations (PNs, $\mathrm{n}=5317$ odor deliveries; ORNs, $\mathrm{n}=2704$ odor deliveries). A linear logistic classifier was trained to predict the known odor identity using 2-fold crossvalidation. That is, a model was trained on half the data and evaluated on the remaining half, and then this process was repeated with the train and test half reversed. The decoding accuracy was quantified as the fraction of odor deliveries in which the predicted odor was correct. 
bioRxiv preprint doi: https://doi.org/10.1101/2021.12.24.474127; this version posted December 24,2021 . The copyright holder for this preprint (which was not certified by peer review) is the author/funder, who has granted bioRxiv a license to display the preprint in perpetuity. It is made available under aCC-BY 4.0 International license.

Churgin \& Lavrentovich et al., 2021 - preprint version - www.biorxiv.org

\section{DoOR data}

DoOR data for the glomeruli and odors relevant to our study was downloaded from http://neuro.uni-konstanz.de/DoOR/default.$h$ tml $^{37}$.

\section{Yoked odor experience experiments}

We selected six flies for which both odor preference and neural activity were recorded to serve as the basis for imposed odor experiences for yoked control flies. The experimental flies were chosen to represent a diversity of preference scores. Each experimental fly's odor experience was binned into discrete odor bouts to represent experience of either $\mathrm{MCH}$ or OCT based on its location in the tunnel as a function of time (Figure 2j). Odor bouts lasting less than $100 \mathrm{~ms}$ were omitted due to limitations on odor-switching capabilities of the odor delivery apparatus. To deliver a given experimental fly's odor experience to yoked controls, we set both odor streams (on either end of the tunnel apparatus) to deliver the same odor experienced by the experimental fly at that moment during the odor-on period. No odor was delivered to yoked controls during time points in which the experimental fly resided in the tunnel choice zone (central $5 \mathrm{~mm}$ ). See Figure $2 \mathrm{j}$ for an example pair of experimental fly and yoked control behavior and odor experience.

\section{Immunohistochemistry}

After measuring odor preference behavior, 7-15 day-old flies were anesthetized on ice and brains were dissected in phosphate buffered saline (PBS). Dissection and immunohistochemistry were carried out as previously reported ${ }^{59}$. The experimenter was blind to the behavioral scores of all individuals throughout dissection, imaging, and analysis. Individual identities were maintained by fixing, washing, and staining each brain in an individual $0.2 \mathrm{~mL}$ PCR tube using fluid volumes of $100 \mathrm{uL}$ per brain (Fisher Scientific). Primary incubation solution contained mouse anti-nc82 (1:40, DSHB), chicken anti-GFP (1:1000, Aves Labs), rabbit anti-mStrawberry $(1: 1000$, biorbyt), and $5 \%$ normal goat serum (NGS, Invitrogen) in PBT (0.5\% Triton X-100 in PBS). Secondary incubation solution contained Atto $647 \mathrm{~N}$-conjugated goat anti-mouse (1:250, Millipore Sigma), Alexa Fluor 568-conjugated goat anti-rabbit (1:250), Alexa Fluor 488-conjugated goat anti-chicken (1:250, ThermoFisher), and 5\% NGS in PBT. Primary and secondary incubation times were 2 and 3 overnights, respectively, at $4^{\circ} \mathrm{C}$. Stained samples were mounted and cleared in Vectashield (H-1000, Vector Laboratories) between two coverslips (12-568B, Fisher Scientific). Two reinforcement labels (5720, Avery) were stacked to create a $0.15 \mathrm{~mm}$ spacer.

\section{Expansion microscopy}

Immunohistochemistry for expansion microscopy was carried out as described above, with the exception that antibody concentrations were modified as follows: mouse anti-nc82 (1:40), chicken anti-GFP (1:200), rabbit anti-mStrawberry (1:200), Atto $647 \mathrm{~N}$-conjugated goat anti-mouse (1:100), Alexa Fluor 568-conjugated goat anti-rabbit (1:100), Alexa Fluor 488-conjugated goat anti-chicken (1:100). Expansion of stained samples was performed as previously described ${ }^{32,33}$. Expanded samples were mounted in coverslip-bottom petri dishes (MatTek Corporation) and anchored by treating the coverslip with poly-l-lysine solution (Millipore Sigma) as previously described ${ }^{33}$.

\section{Confocal imaging}

All confocal imaging was carried out at the Harvard Center for Biological Imaging. Unexpanded samples were imaged on an LSM700 (Zeiss) inverted confocal microscope equipped with a 40x oil-immersion objective (1.3 NA, EC Plan Neofluar, Zeiss). Expanded samples were imaged on an LSM880 (Zeiss) inverted confocal microscope equipped with a $40 \mathrm{x}$ water-immersion objective (1.1 NA, LD C-Apochromat, Zeiss). Acquisition of Zstacks was automated with Zen Black software (Zeiss).

\section{Standard confocal image analysis}

We used custom semi-automated code to generate glomerular segmentation masks from confocal z-stacks of unexpanded Orco $>$ Brp-Short brains. Using Matlab, each image channel was median filtered $\left(\sigma_{x}, \sigma_{y}, \sigma_{z}=11,11,1\right.$ pixels $)$ and downsampled in $\mathrm{x}$ and $\mathrm{y}$ by a factor of 11 . Next, an ORN mask was generated by multiplying and thresholding the Orco $>\mathrm{mCD} 8$ and Orco $>$ BrpShort channels. Next, a locally normalized nc82 and Orco $>\mathrm{mCD} 8$ image stack were multiplied and thresholded, and the ORN mask was applied to remove background and other undesired brain structures. This pipeline resulted in a binary image stack which maximized the contrast of the glomerular structure of the antennal lobe. We then applied a binary distance transform and watershed transform to generate discrete subregions which aimed to represent segmentation masks for each glomerulus tagged by Orco-Gal4.

However, this procedure generally resulted in some degree of under-segmentation; that is, some glomerular segmentation masks were merged. To split each merged segmentation mask, we convolved a ball (whose radius was proportional to the cube root of the volume of the segmentation mask in question) across the mask and thresholded the resulting image. The rationale of this procedure was that 2 merged glomeruli would exhibit a mask shape resembling two touching spheres, and convolving a similarly-sized sphere across this volume followed by thresholding would split the merged object. After ball convolution, we repeated the distance and watershed transform to once more generate discrete subregions representing glomerular segmentation masks. This second watershed step generally resulted in over-segmentation; that is, by visual inspection it was apparent that many glomeruli were split into multiple subregions. Therefore, we finally manually agglomerated the over-segmented subregions to generate single segmentation masks for each glomerulus of interest. We used a published atlas to aid manual identification of glomeruli24. The total Brp-Short fluorescence signal within each glomerulus was determined and divided by the volume of the glomerulus' segmentation mask to calculate BrpShort density values.

\section{Expansion microscopy image analysis}

The spots function in Imaris 9.0 (Bitplane) was used to identify individual Brp-Short puncta in expanded sample image stacks of Or13a $>$ Brp-Short samples ${ }^{31}$. The spot size was set to $0.5 \mathrm{um}$, background subtraction and region-growing were enabled, and the default spot quality threshold was used for each image stack. 
Churgin \& Lavrentovich et al., 2021 - preprint version - www.biorxiv.org

Identified spots were used to mask the Brp-Short channel and the resultant image was saved as a new stack. In Matlab, a glomerular mask was generated by smoothing $\left(\sigma_{x}, \sigma_{y}, \sigma_{z}=40\right.$, 40, 8 pixels) and thresholding (92.5th percentile) the raw BrpShort image stack. The mask was then applied to the spot image stack to remove background spots. Finally, the masked spot image stack was binarized and spot number and properties were quantified.

\section{Antennal Lobe modeling}

We constructed a model of the antennal lobe to test the effect of circuit variation on PN activity variation across individuals. Our general approach to producing realistic circuit activity with the AL model was 1) using experimentally-measured parameters whenever possible (principally the connectome wiring diagram and biophysical parameters measured electrophysiologically), 2) associating free parameters only with biologically plausible categories of elements, while minimizing their number, and 3) tuning the model using those free parameters so that it reproduced high-level patterns of activity considered in the field to represent the canonical operations of the AL. Simulations were run in Python (version 3.6) ${ }^{59}$, and model outputs were analyzed using Jupyter notebooks ${ }^{60}$ and Python and Matlab scripts.

\section{AL model neurons}

Release 1.2 of the hemibrain connectomics dataset ${ }^{34}$ was used to set the connections in the model. Hemibrain body IDs for ORNs, LNs, and PNs were obtained via the lists of neurons supplied in the supplementary tables in ${ }^{61}$. ORNs and PNs of non-olfactory glomeruli (VP1d, VP11, VP1m, VP2, VP3, VP4, VP5) were ignored, leaving 51 glomeruli. Synaptic connections between the remaining 2574 ORNs, $197 \mathrm{LNs}, 166 \mathrm{mPNs}$, and $130 \mathrm{uPNs}$ were queried from the hemibrain API. All ORNs were assigned to be excitatory ${ }^{62}$. Polarities were assigned to PNs based on the neurotransmitter assignments in ${ }^{63}$. mPNs without neurotransmitter information were randomly assigned an excitatory polarity with probability equal to the fraction of neurotransmitter-identified mPNs that are cholinergic; the same process was performed for uPNs. After confirming that the model's output was qualitatively robust to which mPNs and uPNs were randomly chosen, this random assignment was performed once and then frozen for subsequent analyses.

Of the 197 LNs, we assigned 31 to be excitatory, based on the estimated 1:5.4 ratio of eLNs to iLNs in the $\mathrm{AL}^{64}$. To account for observations that eLNs broadly innervate the $\mathrm{AL}^{65}$, all LNs were ranked by the number of innervated glomeruli, and the 31 eLNs were chosen uniformly at random from the top $50 \%$ of LNs in the list. This produced a distribution of glomerular innervations in eLNs qualitatively similar to that of krasavietz LNs in Supplementary Fig 6 of ${ }^{21}$.

\section{Voltage model}

We used a single-compartment leaky-integrate-and-fire voltage model for all neurons as in ${ }^{35}$, in which each neuron had a voltage $V_{i}(t)$ and current $I_{i}(t)$. When the voltage of neuron $i$ was beneath its threshold $V_{i, t h r}$, the following dynamics were obeyed:

$$
C_{i} \frac{d V_{i}}{d t}=\frac{V_{i, 0}-V_{i}(t)}{R_{i}}+I_{i, \text { odor }}(t)+\sum_{j=1}^{N} a_{i} W{ }_{j i} I_{j}(t)
$$

Each neuron $i$ had electrical properties: membrane capacitance $C_{i}$, resistance $R_{i}$, and resting membrane potential $V_{i, 0}$ with values from electrophysiology measurements (Table 3 ).

When the voltage of a neuron exceeded the threshold $V_{i, t h r}$, a templated action potential was filled into its voltage time trace, and a templated postsynaptic current was added to all downstream neurons, following the definitions in ${ }^{35}$.

Odor stimuli were simulated by triggering ORNs to spike at frequencies matching known olfactory receptor responses to the desired odor. The timing of odor-evoked spikes was given by a Poisson process, with firing rate $F R$ for ORNs of a given glomerulus governed by:

$$
F R_{\text {glom, odor }}(t)=F R_{\max } D_{\text {glom }, \text { odor }}\left(f_{a}+\left(1-f_{a}\right) e^{-t / t_{a}}\right)
$$

$F R_{\max }$, the maximum ORN firing rate, was set to $400 \mathrm{~Hz}$. $D_{\text {glom }}$, odor is a value between 0 and 1 from the DoOR database, representing the response of an odorant receptor/glomerulus to an odor, estimated from electrophysiology and/or fluorescence data $^{37}$. ORNs display adaptation to odor stimuli ${ }^{2}$, captured by the final term with timescale $t_{a}=110 \mathrm{~ms}$ to $75 \%$ of the initial value, as done in ${ }^{6}$. Thus, the functional maximum firing rate of an ORN was $75 \%$ of $400 \mathrm{~Hz}=300 \mathrm{~Hz}$, matching the highest ORN firing rates observed experimentally ${ }^{67}$. After determining the times of ORN spikes according to this firing-rate rule, spikes were induced by the addition of $10^{6}$ picoamps in a single time step. This reliably triggered an action potential in the ORN, regardless of currents from other neurons. In the absence of odors, spike times for ORNs were drawn by a Poisson process at $10 \mathrm{~Hz}$, to match reported spontaneous firing rates 68 .

For odor-glomeruli combinations with missing DoOR values ( $40 \%$ of the dataset), we performed imputation via alternating least squares (using the pca function with option 'als' to infill missing values ${ }^{69}$ ) on the odor $\mathrm{x}$ glomerulus matrix 1000 times and taking the mean infilled matrix, which provides a closer match to ground truth missing values than a single run of ALS (Figure S4 of ${ }^{70}$ ).

A neuron $j$ presynaptic to $i$ supplies its current $I_{j}(t)$ scaled by the synapse strength $W_{j i}$, the number of synapses in the hemibrain dataset from neuron $j$ to $i$. Rows in $W$ corresponding to neurons with inhibitory polarity (i.e. GABAergic PNs or LNs) were set negative. Finally, post-synaptic neurons (columns of the connectivity matrix) have a class-specific multiplier $a_{i}$, a hand-tuned value, described below.

\section{AL model tuning}

Class-specific multiplier current multipliers $\left(a_{i}\right)$ were tuned using the panel of 18 odors from ${ }^{18}$ (our source for several experi ${ }^{-}$ mental observations of high-level AL function): benzaldehyde, butyric acid, 2,3-butanedione, 1-butanol, cyclohexanone, Z3hexenol, ethyl butyrate, ethyl acetate, geranyl acetate, isopentyl acetate, isoamyl acetate, 4-methylphenol, methyl salicylate, 3methylthio-1-propanol, octanal, 2-octanone, pentyl acetate, E2hexenal, trans-2-hexenal, gamma-valerolactone. Odors were 
Churgin \& Lavrentovich et al., 2021 - preprint version - www.biorxiv.org

"administered" for $400 \mathrm{~ms}$ each, with $300 \mathrm{~ms}$ odor-free pauses between odor stimuli.

The high-level functions of the $\mathrm{AL}$ that represent a baseline, working condition were: (1) firing rates for ORNs, LNs, and PNs matching the literature (listed in Table $318,38-40$, (2) a more uniform distribution of PN firing rates during odor stimuli compared to ORN firing rates, (3) greater separation of representations of odors in PN-coding space than in ORN-coding space, and (4) a sublinear transfer function between ORN firing rates and PN firing rates. Features (2) - (4) relate to the role of the AL in enhancing the separability of similar odors ${ }^{18}$.

To find a parameterization with those functions, we tuned the values of $a_{i}$ as scalar multipliers on ORN, eLN, iLN, and PN columns of the hemibrain connectivity matrix. Thus, these values represent cell type-specific sensitivities to presynaptic currents, which may be justified by the fact that ORNs/LNs/PNs are genetically distinct cell populations ${ }^{71,72}$. A small grid search of the four class-wise sensitivity parameters produced a configuration that reasonably satisfied the above criteria (Fig S14). In this configuration, the ORN columns of the hemibrain connectivity matrix are scaled by 0.1 , eLNs by 0.04 , iLNs by 0.02 , and PNs by 0.4 . The relatively large multiplier on PNs is potentially consistent with the fact that PNs are sensitive to small differences between weak ORN inputs ${ }^{18}$. Model outputs were robust over several different sets of $a_{i}$, provided iLN sensitivity $\simeq \mathrm{eLN}<$ ORN $<$ PN.

Notable ways in which the model behavior deviates from experimental recordings (and thus caveats on the interpretation of the model) include: 1) Model LNs appear to have more heterogeneous firing rates than real LNs, with many LNs inactive for this panel of odor stimuli. This likely reflects a lack of plastic/homeostatic mechanisms in the model to regularize $\mathrm{LN}$ firing rates given their variable synaptic connectivity ${ }^{21}$. 2) Some PNs had off-odor rates that are high compared to real PNs, resulting in a distribution of ON-OFF responses that had a lower limit than in real recordings. Qualitatively close matches were achieved between the model and experimental data in the distributions of odor representations in ORN vs PN spaces and the non-linearity of the ORN-PN transfer function.

\section{AL model circuit variation generation}

We generated AL circuit variability in two ways: cell-type bootstrapping, and synapse density resampling. These methods assume that the distribution of circuit configurations across individual ALs can be generated by resampling circuit components within a single individual's AL (neurons and glomerular synaptic densities, respectively, from the hemibrain EM volume).

To test the effect of variation in the developmental complement of neurons of particular types, we bootstrapped populations of interest from the list of hemibrain neurons. Resampling with replacement of ORNs was performed glomerulus-by-glomerulus, i.e., separately among each pool of ORNs expressing a particular Odorant receptor gene. The same was done for PNs. For LNs, all 197 LNs were treated as a single pool; there was no finer operation based on LN subtypes or glomerular innervations. This choice reflects the high developmental variability of
$\mathrm{LNs}^{21}$. The number of synapses between a pair of bootstrapped neurons was equal to the synapse count between those neurons in the hemibrain connectivity matrix.

In some glomeruli, bootstrapping PNs produced unreasonably high variance in the total PN synapse count. For instance, DP1m, DC4, and DM3 each harbor PNs that differ in total synapse count by a factor of $\sim 10$. Since these glomeruli have between two to three PNs each, in a sizable proportion of bootstrap samples, all-highly connected (or all-lowly) connected PNs are chosen in such glomeruli. To remedy this biologically unrealistic outcome, we examined the relationship between total input PN synapses within a glomerulus and glomerular volume (Fig S15). In the "synapse density resampling" method, we required that the number of PN input synapses within a glomerulus reflect a draw from the empirical relationship between total input PN synapses and glomerular volume as present in the hemibrain data set. This was achieved by, for each glomerulus, sampling from the following distribution that depends on glomerular volume, then multiplying the number of PN input synapses by a scalar to match that sampled value:

$$
\log S_{g}=\log \left(a V_{g}^{d}\right)+\varepsilon_{g^{\prime}} \varepsilon_{g} \sim N\left(0, \sigma^{2}\right)
$$

Here $S_{g}$ is the PN input synapse count for glomerulus $g, V_{g}$ is the volume of glomerulus $g$ (in cubic microns), $\varepsilon$ is a Gaussian noise variable with standard deviation $\sigma$, and $a, d$ are the scaling factor and exponent of the volume term, respectively. The values of these parameters ( $a=8.98, d=0.73, \sigma=0.38)$ were fit using maximum likelihood.

\section{Acknowledgements}

We thank Asa Barth-Maron and Rachel Wilson for discussions helpful to the AL modeling, and Katrin Vogt for help revising the manuscript. Ed Soucy and Brett Graham of the Center for Brain Science Neuroengineering Core helped maintain the olfactometer and microscope. D.L. was supported by Harvard's Quantitative Biology Initiative. B.L.d.B. was supported by a Klingenstein-Simons Fellowship Award, a Smith Family Odyssey Award, a Harvard/MIT Basic Neuroscience Grant, National Science Foundation grant no. IOS-1557913, and NIH/NINDS grant no. 1R01NS121874-01. E.S.B. was supported by a Harvard/MIT Basic Neuroscience Grant, Lisa Yang, John Doerr, and NIH grant no. 1R01EB024261.

\section{Conflicts of Interest}

The authors declare no competing interests.

\section{References}

1. Ayroles, J. F. et al. Behavioral idiosyncrasy reveals genetic control of phenotypic variability. Proc. Natl. Acad. Sci. U. S. A. 112, 6706-6711 (2015). 
bioRxiv preprint doi: https://doi.org/10.1101/2021.12.24.474127; this version posted December 24, 2021. The copyright holder for this preprint (which was not certified by peer review) is the author/funder, who has granted bioRxiv a license to display the preprint in perpetuity. It is made available under aCC-BY 4.0 International license.

Churgin \& Lavrentovich et al., 2021 - preprint version - www.biorxiv.org

2. Stern, S., Kirst, C. \& Bargmann, C. I. Neuromodulatory Control of Long-Term Behavioral Patterns and Individuality across Development. Cell 171, 1649-1662.e10 (2017).

3. Pantoja, C. et al. Neuromodulatory Regulation of Behavioral Individuality in Zebrafish. Neuron 91, 587-601 (2016).

4. Honegger, K. S., Smith, M. A.-Y., Churgin, M. A., Turner, G. C. \& de Bivort, B. L. Idiosyncratic neural coding and neuromodulation of olfactory individuality in. Proc. Natl. Acad. Sci. U. S. A. (2019) doi:10.1073/pnas.1901623116.

5. Renart, A. \& Machens, C. K. Variability in neural activity and behavior. Current Opinion in Neurobiology vol. 25 211220 (2014).

6. Freund, J. et al. Emergence of individuality in genetically identical mice. Science 340, 756-759 (2013).

7. Bierbach, D., Laskowski, K. L. \& Wolf, M. Behavioural individuality in clonal fish arises despite near-identical rearing conditions. Nat. Commun. 8, 15361 (2017).

8. Westneat, D. F., Wright, J. \& Dingemanse, N. J. The biology hidden inside residual within-individual phenotypic variation. Biol. Rev. Camb. Philos. Soc. 90, 729-743 (2015).

9. Marder, E. Variability, compensation, and modulation in neurons and circuits. Proc. Natl. Acad. Sci. U. S. A. 108 Suppl 3, 15542-15548 (2011).

10.Avitan, L. et al. Behavioral Signatures of a Developing Neural Code. Curr. Biol. 30, 3352-3363.e5 (2020).

11.Linneweber, G. A. et al. A neurodevelopmental origin of behavioral individuality in the Drosophila visual system. Science 367, 1112-1119 (2020).

12.Skutt-Kakaria, K., Reimers, P., Currier, T. A., Werkhoven, Z. $\&$ de Bivort, B. L. A neural circuit basis for context-modulation of individual locomotor behavior. bioRxiv (2019) doi:10.1101/797126.

13.Wang, J. W., Wong, A. M., Flores, J., Vosshall, L. B. \& Axel, $\mathrm{R}$. Two-photon calcium imaging reveals an odor-evoked map of activity in the fly brain. Cell 112, 271-282 (2003).

14.Vosshall, L. B., Wong, A. M. \& Axel, R. An olfactory sensory map in the fly brain. Cell 102, 147-159 (2000).

15.Wilson, R. I. Transformation of Olfactory Representations in the Drosophila Antennal Lobe. Science vol. 303 366-370 (2004).

16.Groschner, L. N. \& Miesenböck, G. Mechanisms of Sensory Discrimination: Insights from Olfaction. Annu. Rev. Biophys. 48, 209-229 (2019).

17.Wilson, R. I. Early Olfactory Processing in Drosophila: Mechanisms and Principles. Annual Review of Neuroscience vol. 36 217-241 (2013).

18.Bhandawat, V., Olsen, S. R., Gouwens, N. W., Schlief, M. L. \& Wilson, R. I. Sensory processing in the Drosophila antennal lobe increases reliability and separability of ensemble odor representations. Nat. Neurosci. 10, 1474-1482 (2007).

19.Couto, A., Alenius, M. \& Dickson, B. J. Molecular, anatomical, and functional organization of the Drosophila olfactory system. Curr. Biol. 15, 1535-1547 (2005).

20.Tobin, W. F., Wilson, R. I. \& Lee, W.-C. A. Wiring variations that enable and constrain neural computation in a sensory microcircuit. eLife 6, e24838 (2017).

21.Chou, Y.-H. et al. Diversity and wiring variability of olfactory local interneurons in the Drosophila antennal lobe. Nat. Neurosci. 13, 439-449 (2010).

22.Chen, T.-W. et al. Ultrasensitive fluorescent proteins for imaging neuronal activity. Nature 499, 295-300 (2013).

23.Alisch, T., Crall, J. D., Kao, A. B., Zucker, D. \& de Bivort, B. L. MAPLE (modular automated platform for large-scale experiments), a robot for integrated organism-handling and phenotyping. eLife 7, (2018).

24.Grabe, V., Strutz, A., Baschwitz, A., Hansson, B. S. \& Sachse, S. Digital in vivo 3D atlas of the antennal lobe of
Drosophila melanogaster. J. Comp. Neurol. 523, 530-544 (2015).

25.Osborne, L. C., Lisberger, S. G. \& Bialek, W. A sensory source for motor variation. Nature 437, 412-416 (2005).

26.Michelson, C. A., Pillow, J. W. \& Seidemann, E. Majority of choice-related variability in perceptual decisions is present in early sensory cortex. bioRxiv (2017) doi: $10.1101 / 207357$.

27.Sachse, S. et al. Activity-dependent plasticity in an olfactory circuit. Neuron 56, 838-850 (2007).

28.Golovin, R. M. \& Broadie, K. Developmental experiencedependent plasticity in the first synapse of the Drosophila olfactory circuit. J. Neurophysiol. 116, 2730-2738 (2016).

29.Iyengar, A., Chakraborty, T. S., Goswami, S. P., Wu, C.-F. \& Siddiqi, O. Post-eclosion odor experience modifies olfactory receptor neuron coding in Drosophila. Proc. Natl. Acad. Sci. U. S. A. 107, 9855-9860 (2010).

30.Mazor, O. \& Laurent, G. Transient dynamics versus fixed points in odor representations by locust antennal lobe projection neurons. Neuron 48, 661-673 (2005).

31.Mosca, T. J. \& Luo, L. Synaptic organization of the Drosophila antennal lobe and its regulation by the Teneurins. eLife vol. 3 (2014).

32.Gao, R. et al. Cortical column and whole-brain imaging with molecular contrast and nanoscale resolution. Science $\mathbf{3 6 3}$, (2019).

33.Asano, S. M. et al. Expansion Microscopy: Protocols for Imaging Proteins and RNA in Cells and Tissues. Curr. Protoc. Cell Biol. 80, e56 (2018).

34.Scheffer, L. K. et al. A connectome and analysis of the adult Drosophila central brain. eLife 9, (2020).

35.Kakaria, K. S. \& de Bivort, B. L. Ring Attractor Dynamics Emerge from a Spiking Model of the Entire Protocerebral Bridge. Front. Behav. Neurosci. 11, 8 (2017).

36.Pisokas, I., Heinze, S. \& Webb, B. The head direction circuit of two insect species. eLife 9, (2020).

37.Münch, D. \& Galizia, C. G. DoOR 2.0--Comprehensive Mapping of Drosophila melanogaster Odorant Responses. Sci. Rep. 6, 21841 (2016).

38.Adrienne E. Dubin, G. L. H. Voltage-activated and odormodulated conductances in olfactory neurons of Drosophila melanogaster. Journal of Neurobiology 32, 123-137 (1997).

39.Seki, Y., Rybak, J., Wicher, D., Sachse, S. \& Hansson, B. S. Physiological and morphological characterization of local interneurons in the Drosophila antennal lobe. J. Neurophysiol. 104, 1007-1019 (2010).

40.Jeanne, J. M. \& Wilson, R. I. Convergence, Divergence, and Reconvergence in a Feedforward Network Improves Neural Speed and Accuracy. Neuron 88, 1014-1026 (2015).

41.Newsome, W. T., Britten, K. H. \& Anthony Movshon, J. Neuronal correlates of a perceptual decision. Nature vol. 341 52-54 (1989).

42.Britten, K. H., Newsome, W. T., Shadlen, M. N., Celebrini, S. \& Movshon, J. A. A relationship between behavioral choice and the visual responses of neurons in macaque MT. Vis. Neurosci. 13, 87-100 (1996).

43.Mellert, D. J., Williamson, W. R., Shirangi, T. R., Card, G. M. \& Truman, J. W. Genetic and Environmental Control of Neurodevelopmental Robustness in Drosophila. PLoS One 11, e0155957 (2016).

44.Liu, T. X., Davoudian, P. A., Lizbinski, K. M. \& Jeanne, J. M. Connectomic features underlying diverse synaptic connection strengths and subcellular computation. bioRxiv 2021.08.19.456845 (2021) doi:10.1101/2021.08.19.456845.

45.Nagel, K. I., Hong, E. J. \& Wilson, R. I. Synaptic and circuit mechanisms promoting broadband transmission of olfactory stimulus dynamics. Nat. Neurosci. 18, 56-65 (2015). 
bioRxiv preprint doi: https://doi.org/10.1101/2021.12.24.474127; this version posted December 24,2021 . The copyright holder for this preprint (which was not certified by peer review) is the author/funder, who has granted bioRxiv a license to display the preprint in perpetuity. It is made available under aCC-BY 4.0 International license.

Churgin \& Lavrentovich et al., 2021 - preprint version - www.biorxiv.org

46.Körholz, J. C. et al. Selective increases in inter-individual variability in response to environmental enrichment in female mice. eLife 7, (2018).

47.Akhund-Zade, J., Ho, S., O'Leary, C. \& de Bivort, B. The effect of environmental enrichment on behavioral variability depends on genotype, behavior, and type of enrichment. J. Exp. Biol. 222, (2019).

48.Zocher, S. et al. Early-life environmental enrichment generates persistent individualized behavior in mice. Sci Adv 6 , eabb1478 (2020).

49.Honegger, K. \& de Bivort, B. Stochasticity, individuality and behavior. Curr. Biol. 28, R8-R12 (2018).

50.Hopper, K. R. Risk-spreading and bet-hedging in insect population biology. Annu. Rev. Entomol. 44, 535-560 (1999).

51.Akhund-Zade, J. et al. Wild flies hedge their thermal preference bets in response to seasonal fluctuations. bioRxiv 2020.09.16.300731 (2020) doi:10.1101/2020.09.16.300731.

52.Kain, J. S. et al. Variability in thermal and phototactic preferences in Drosophila may reflect an adaptive bet-hedging strategy. Evolution 69, 3171-3185 (2015).

53.Krams, I. A. et al. Serotoninergic Modulation of Phototactic Variability Underpins a Bet-Hedging Strategy in Drosophila melanogaster. Front. Behav. Neurosci. 15, 659331 (2021).

54.Gomez-Marin, A. \& Ghazanfar, A. A. The Life of Behavior. Neuron 104, 25-36 (2019).

55.Raj, A., Rifkin, S. A., Andersen, E. \& van Oudenaarden, A. Variability in gene expression underlies incomplete penetrance. Nature 463, 913-918 (2010).

56.Li, H. et al. Classifying Drosophila Olfactory Projection Neuron Subtypes by Single-Cell RNA Sequencing. Cell 171, 1206-1220.e22 (2017).

57.Werkhoven, Z. et al. The structure of behavioral variation within a genotype. eLife 10, e64988 (2021).

58.Wu, J. S. \& Luo, L. A protocol for dissecting Drosophila melanogaster brains for live imaging or immunostaining. Nat. Protoc. 1, 2110-2115 (2006).

59.van Rossum, G. \& Drake, F. L. The Python Language Reference Manual. (Network Theory Ltd., 2011).

60.Kluyver, T. et al. Jupyter Notebooks - a publishing format for reproducible computational workflows. in Positioning and Power in Academic Publishing: Players, Agents and Agendas (eds. Loizides, F. \& Scmidt, B.) 87-90 (IOS Press, 2016).

61.Schlegel, P. et al. Information flow, cell types and stereotypy in a full olfactory connectome. bioRxiv 2020.12.15.401257 (2020) doi:10.1101/2020.12.15.401257.

62.Wilson, R. I. Early olfactory processing in Drosophila: mechanisms and principles. Annu. Rev. Neurosci. 36, 217-241 (2013).

63.Bates, A. S. et al. Complete Connectomic Reconstruction of Olfactory Projection Neurons in the Fly Brain. Curr. Biol. (2020) doi:10.1016/j.cub.2020.06.042.

64.Tsai, K.-T., Hu, C.-K., Li, K.-W., Hwang, W.-L. \& Chou, Y.H. Circuit variability interacts with excitatory-inhibitory diversity of interneurons to regulate network encoding capacity. Sci. Rep. 8, 8027 (2018).

65.Shang, Y., Claridge-Chang, A., Sjulson, L., Pypaert, M. \& Miesenböck, G. Excitatory local circuits and their implications for olfactory processing in the fly antennal lobe. Cell 128, 601-612 (2007).

66.Kao, K.-W. \& Lo, C.-C. Short term depression, presynaptic inhibition and local neuron diversity play key functional roles in the insect antennal lobe. J. Comput. Neurosci. (2020) doi:10.1007/s10827-020-00747-4.

67.Hallem, E. A., Ho, M. G. \& Carlson, J. R. The molecular basis of odor coding in the Drosophila antenna. Cell 117, 965-979 (2004). 68.de Bruyne, M., Foster, K. \& Carlson, J. R. Odor coding in the Drosophila antenna. Neuron 30, 537-552 (2001).

69.MATLAB pca documentation. MathWorks https://www.mathworks.com/help/stats/pca.html.

70.Werkhoven, Z. et al. The structure of behavioral variation within a genotype. bioRxiv 779363 (2020) doi: 10.1101/779363.

71.Xie, Q. et al. Temporal evolution of single-cell transcriptomes of Drosophila olfactory projection neurons. eLife 10, (2021).

72.McLaughlin, C. N. et al. Single-cell transcriptomes of developing and adult olfactory receptor neurons in Drosophila. eLife 10, (2021).

73.Olsen, S. R. \& Wilson, R. I. Lateral presynaptic inhibition mediates gain control in an olfactory circuit. Nature $\mathbf{4 5 2}$, 956-960 (2008).

74.Yaksi, E. \& Wilson, R. I. Electrical coupling between olfactory glomeruli. Neuron 67, 1034-1047 (2010).

75.Luo, S. X., Axel, R. \& Abbott, L. F. Generating sparse and selective third-order responses in the olfactory system of the fly. Proc. Natl. Acad. Sci. U. S. A. 107, 10713-10718 (2010).

76.Rohatgi, A. Webplotdigitizer: Version 4.5. (2021).

77.Cao, L.-H. et al. Distinct signaling of Drosophila chemoreceptors in olfactory sensory neurons. Proc. Natl. Acad. Sci. U.S. A. 113, E902-11 (2016).

78.Wilson, R. I. \& Laurent, G. Role of GABAergic inhibition in shaping odor-evoked spatiotemporal patterns in the Drosophila antennal lobe. J. Neurosci. 25, 9069-9079 (2005).

79.Huang, Y.-C. et al. A Single-Cell Level and Connectome-Derived Computational Model of the Drosophila Brain. Front. Neuroinform. 12, 99 (2018). 
bioRxiv preprint doi: https://doi.org/10.1101/2021.12.24.474127; this version posted December 24,2021 . The copyright holder for this preprint (which was not certified by peer review) is the author/funder, who has granted bioRxiv a license to display the preprint in perpetuity. It is made available under aCC-BY 4.0 International license.

Churgin \& Lavrentovich et al., 2021 - preprint version - www.biorxiv.org

\section{Supplementary materials}

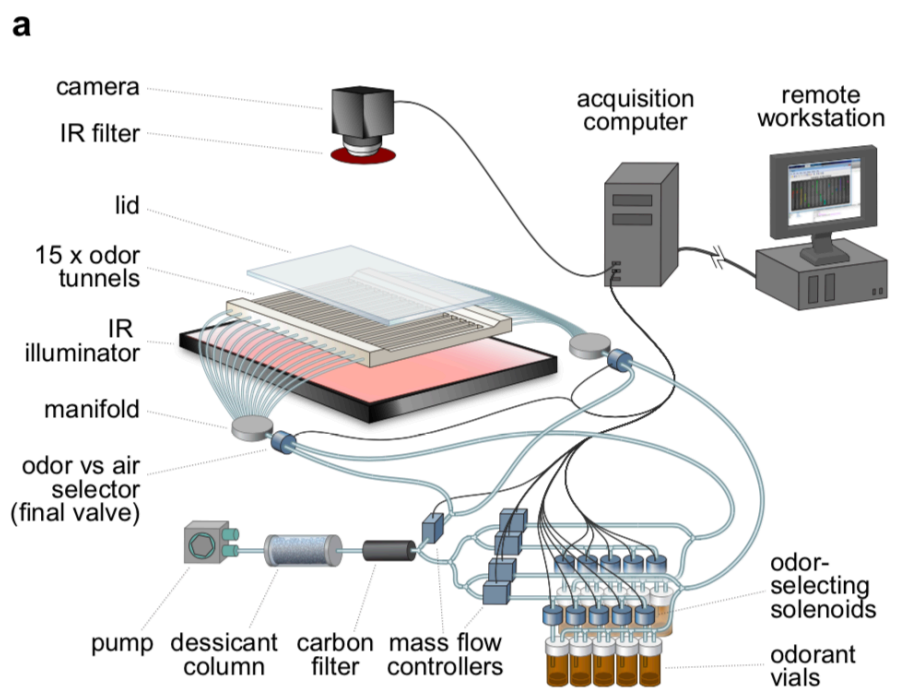

b

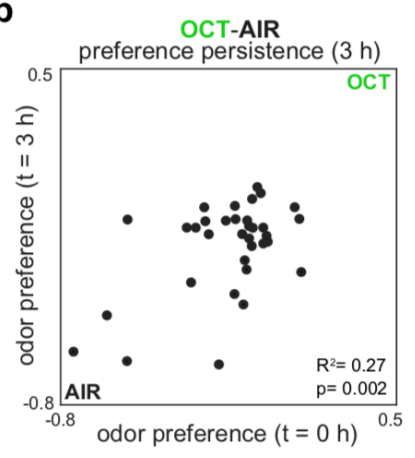

d

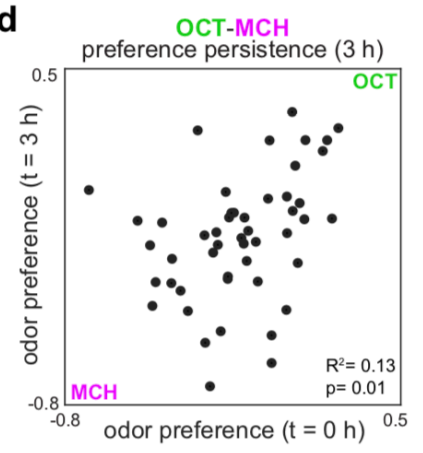

C

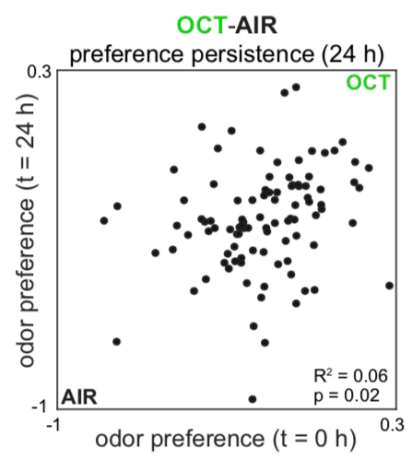

e

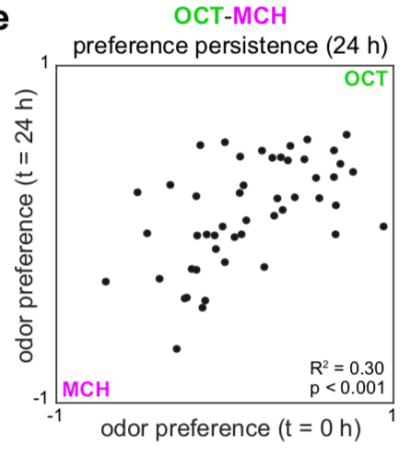

Supplementary Figure 1: Behavioral measurements and individual preference persistence (a) Behavioral measurement apparatus (adapted from ${ }^{4}$ ). (b) Odor preference persistence over 3 hours for flies given a choice between 3-octanol and air ( $\mathrm{n}=34$ flies). (c) Odor preference persistence over 24 hours for flies given a choice between 3-octanol and air ( $\mathrm{n}=97$ flies). (d) Odor preference persistence over 3 hours for flies given a choice between 3-octanol and 4-methylcyclohexanol ( $\mathrm{n}=51 \mathrm{flies})$. (e) Odor preference persistence over 24 hours for flies given a choice between 3 -octanol and 4-methylcyclohexanol ( $\mathrm{n}=49$ flies). 
bioRxiv preprint doi: https://doi.org/10.1101/2021.12.24.474127; this version posted December 24, 2021. The copyright holder for this preprint (which was not certified by peer review) is the author/funder, who has granted bioRxiv a license to display the preprint in perpetuity. It is made available under aCC-BY 4.0 International license.

Churgin \& Lavrentovich et al., 2021 - preprint version - www.biorxiv.org

Glomerulus:

$\underline{\text { Odor }}$

air

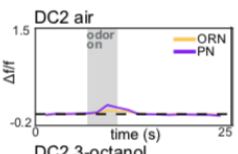

3-octanol

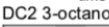

1-hexanol

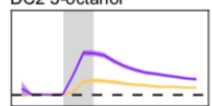

DC2 1-hexanol

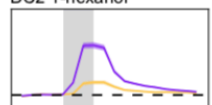

DC2 ethyl lactate

ethyl lactate

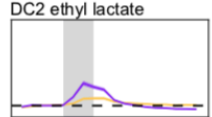

DC2 citronella

citronella

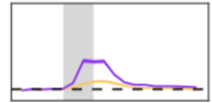

DC2 2-heptanone

2-heptanone

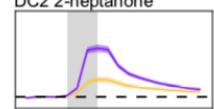

DC2 1-pentano

1-pentanol

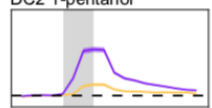

DC2 ethanol

ethanol

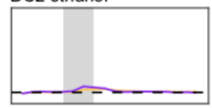

geranyl acetate

$\mathrm{DC} 2$ geranyl acetate

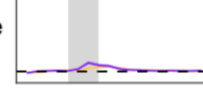

DC2 hexyl acetate

hexyl acetate

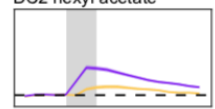

DC2 4-methylcyclohexan

4-methylcyclohexanol

pentyl acetate

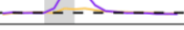

DC2 pentyl acetate

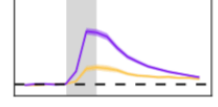

DC2 1-butanol

1-butanol

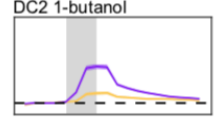

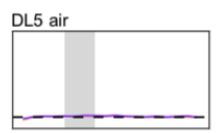

DL5 3-octanol

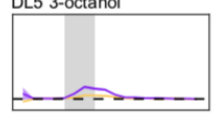

DL5 1-hexanol

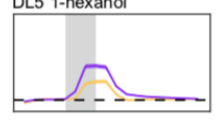

DL5 ethyl lactate

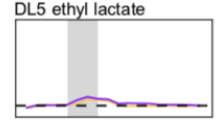

DL5 citronella

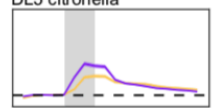

DL5 2-heptanone

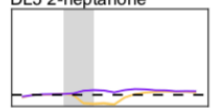

DL5 1-pentanol

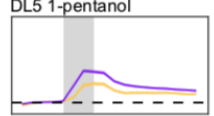

DL5 ethanol

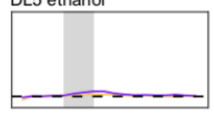

DL5 geranyl acetate

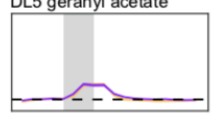

DL5 hexyl acetate

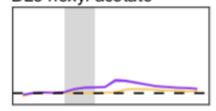

DL5 4-methylcyclohexano

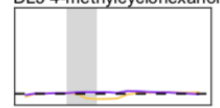

DL5 pentyl acetate

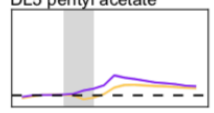

DL5 1-butanol

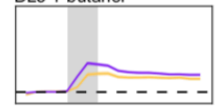

DM1

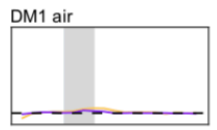

DM1 3-octanol

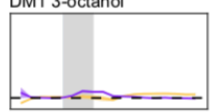

DM1 1-hexanol

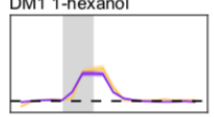

DM1 ethyl lactate

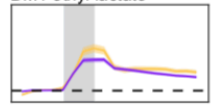

DM1 citronella

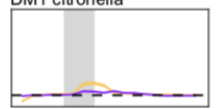

DM1 2-heptanone

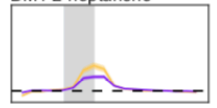

DM1 1-pentanol

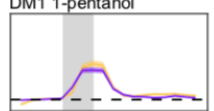

DM1 ethanol

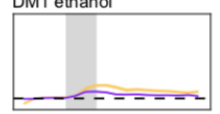

DM1 geranyl acetate

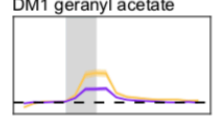

DM1 hexyl acetate

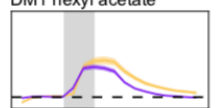

DM1 4-methylcyclohexanol

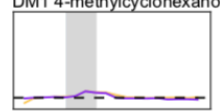

DM1 pentyl acetate

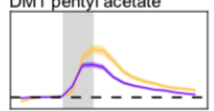

DM1 1-butanol

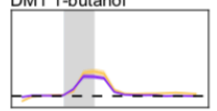

DM2

DM3

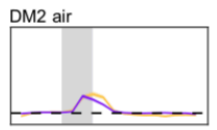

DM2 3-octanol

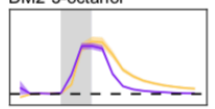

DM2 1-hexanol

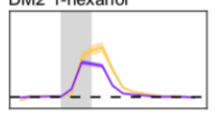

DM2 ethyl lactate
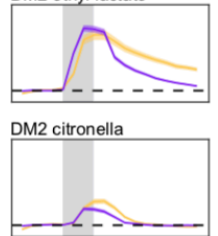

DM2 2-heptanone

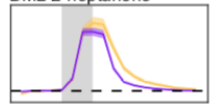

DM2 1-pentanol

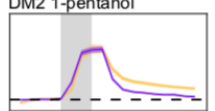

DM2 ethanol

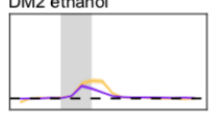

DM2 geranyl acetate

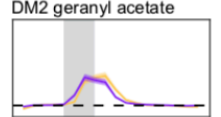

DM2 hexyl acetate

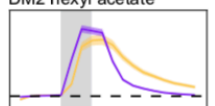

DM2 4-methylcyclohexano

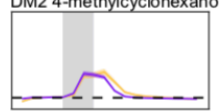

DM2 pentyl acetate

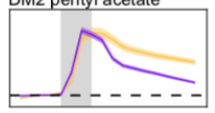

DM2 1-butanol

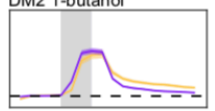

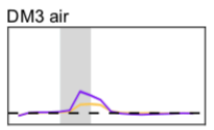

DM3 3-octanol

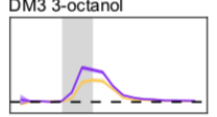

DM3 1-hexanol

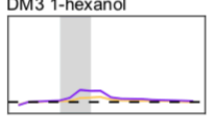

DM3 ethyl lactate
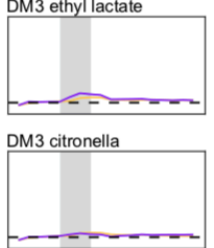

DM3 2-heptanone

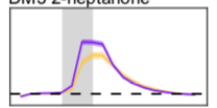

DM3 1-pentano

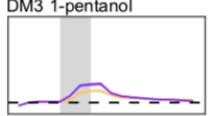

DM3 ethanol

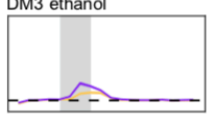

DM3 geranyl acetate

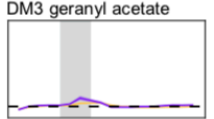

DM3 hexyl acetate

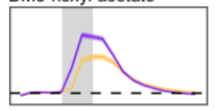

DM3 4-methylcyclohexano

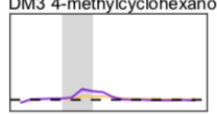

DM3 pentyl acetate

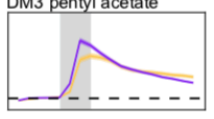

DM3 1-butanol

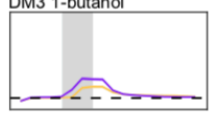

Supplementary Figure 2: Average glomerulus-odor time-dependent responses. Time-dependent responses of each glomerulus identified in our study to the 13 odors in our odor panel. Data represents the average across flies (ORN, peach curves, $n=65$ flies; PN, plum curves, $n=122$ flies). Shaded error bars represent S.E.M. 
bioRxiv preprint doi: https://doi.org/10.1101/2021.12.24.474127; this version posted December 24, 2021. The copyright holder for this preprint (which was not certified by peer review) is the author/funder, who has granted bioRxiv a license to display the preprint in perpetuity. It is made available under aCC-BY 4.0 International license.

Churgin \& Lavrentovich et al., 2021 - preprint version - www.biorxiv.org

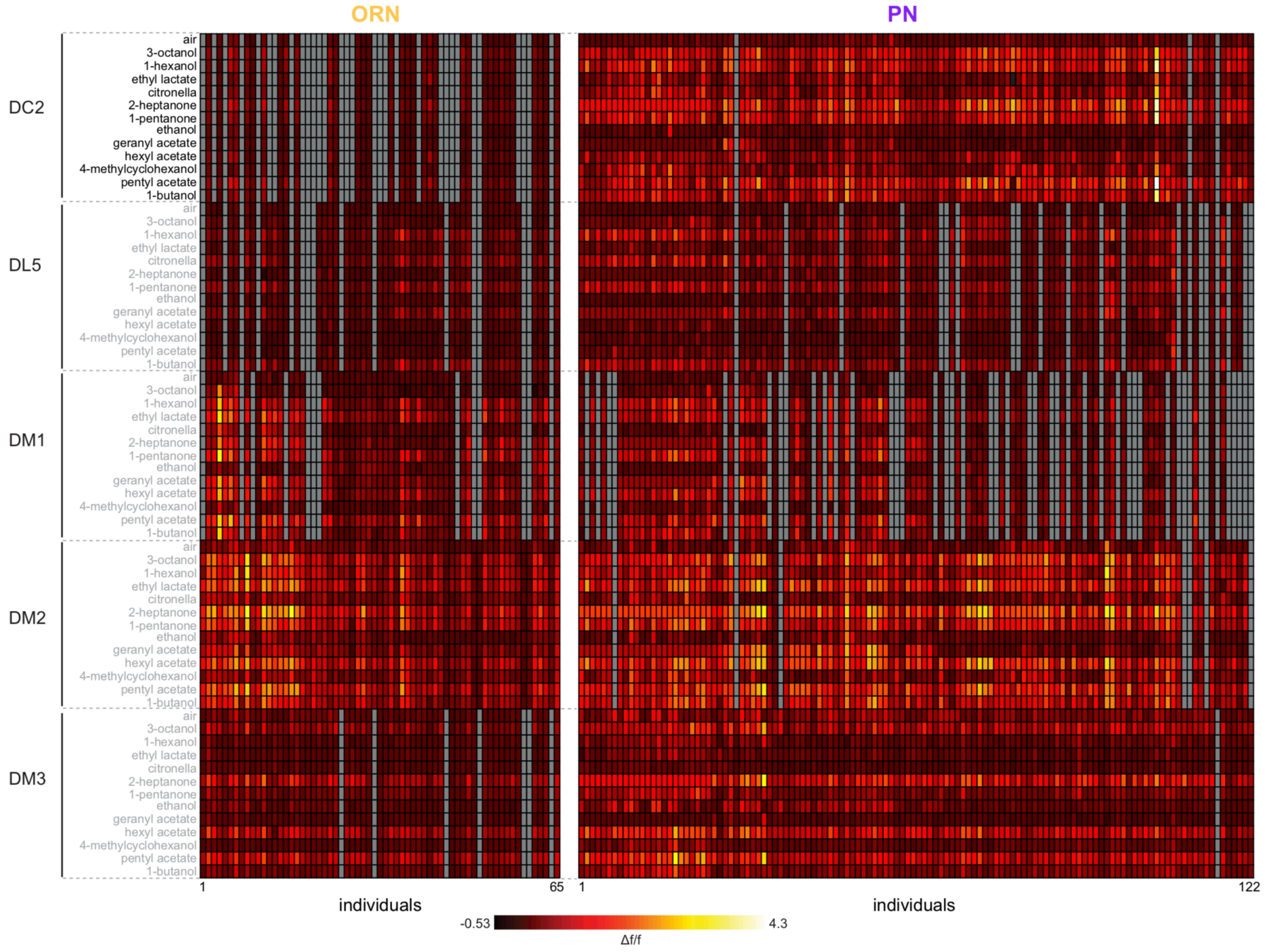

Supplementary Figure 3: Individual glomerulus-odor responses (a) Idiosyncratic odor coding measured in ORNs (left, $\mathrm{n}=65$ flies) and PNs (right, $n=122$ flies). Each column represents the response ( $\max \Delta \mathrm{f} / \mathrm{f}$ attained over the odor trial) of a single fly averaged over up to 4 odor deliveries. Each row represents a glomerulus-odor response pair. Missing data are indicated in gray. 
bioRxiv preprint doi: https://doi.org/10.1101/2021.12.24.474127; this version posted December 24, 2021. The copyright holder for this preprint (which was not certified by peer review) is the author/funder, who has granted bioRxiv a license to display the preprint in perpetuity. It is made available under aCC-BY 4.0 International license.

Churgin \& Lavrentovich et al., 2021 - preprint version - www.biorxiv.org

a

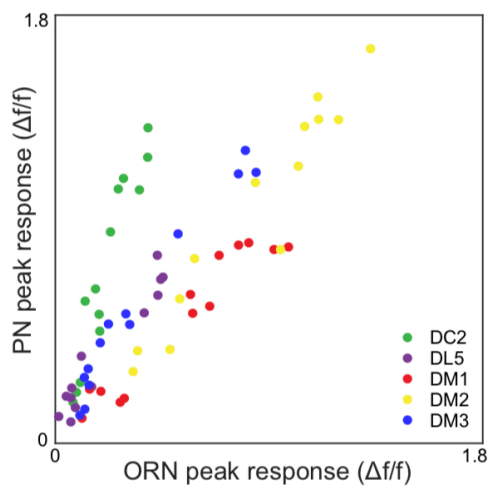

b

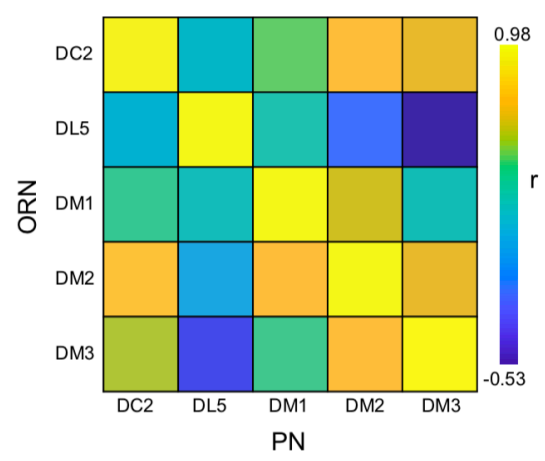

C

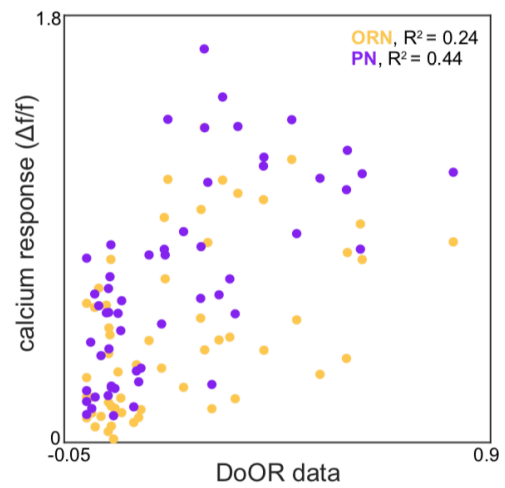

d
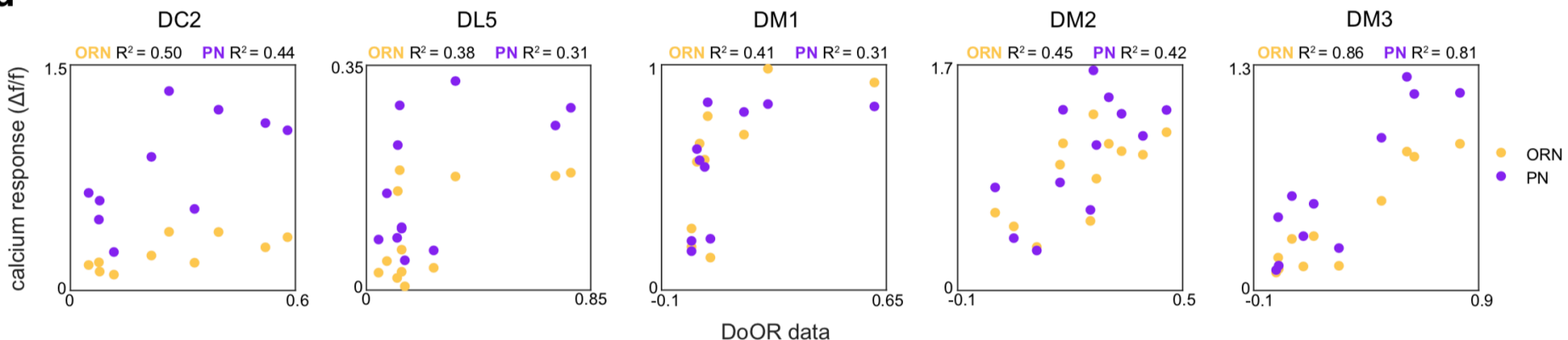

Supplementary Figure 4: Glomerulus responses and identification (a) Glomerulus odor responses measured in PNs versus those measured in ORNs. Points correspond to the odorants listed in Figure 1g. (b) Cross-odor trial correlation matrix between glomerular odor responses in ORNs and PNs. (c) Peak calcium responses for each glomerulus-odor pair measured in this study plotted against those recorded in the DoOR dataset ${ }^{37}$. (d) Peak calcium responses for each individual glomerulus plotted against those recorded in the DoOR dataset. 
bioRxiv preprint doi: https://doi.org/10.1101/2021.12.24.474127; this version posted December 24,2021 . The copyright holder for this preprint (which was not certified by peer review) is the author/funder, who has granted bioRxiv a license to display the preprint in perpetuity. It is made available under aCC-BY 4.0 International license.

Churgin \& Lavrentovich et al., 2021 - preprint version - www.biorxiv.org

a

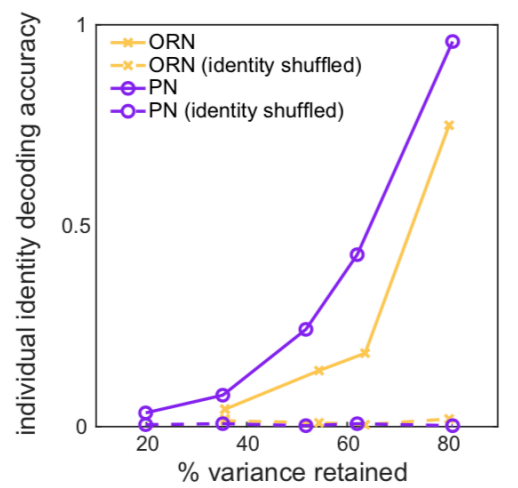

\section{C}

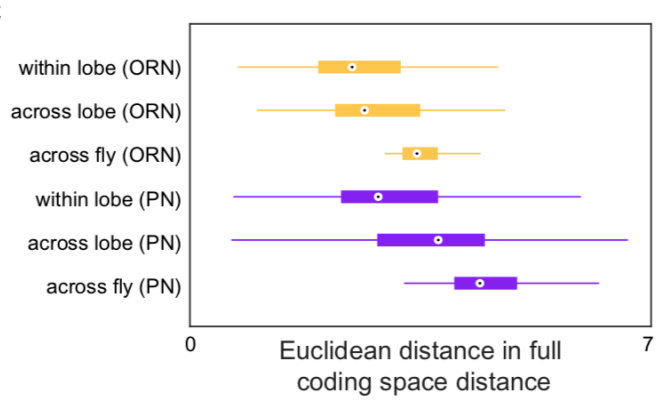

b

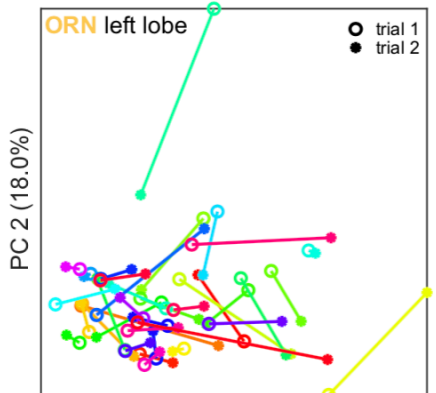

PC $1(50.4 \%)$

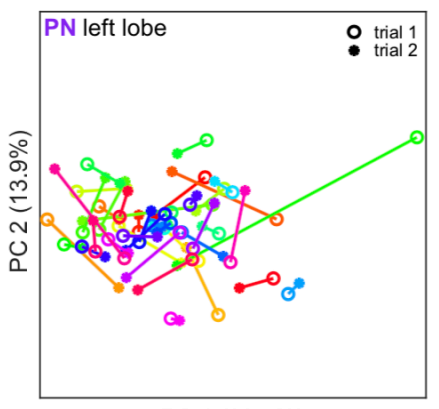

PC $1(30.5 \%)$

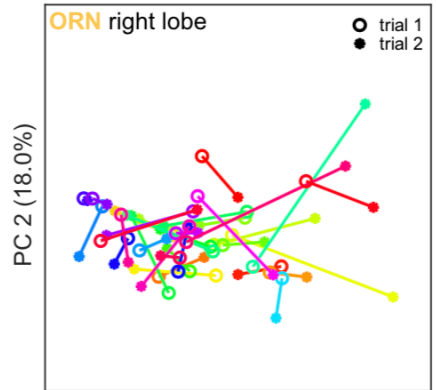

PC $1(50.4 \%)$

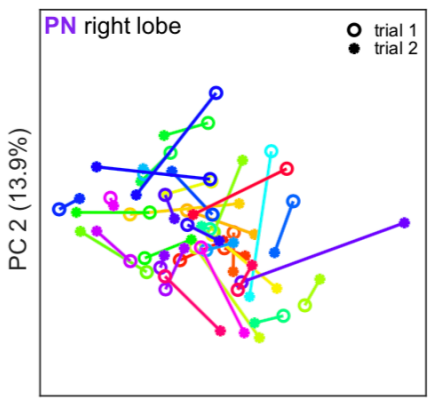

PC $1(30.5 \%)$

Supplementary Figure 5: Idiosyncrasy of ORN and PN responses (a) Logistic regression classifier accuracy of decoding individual identity from individual odor panel peak responses. PCA was performed on population responses and the specified fraction of variance (x-axis) was retained. Individual identity can be better decoded from PN responses than ORN responses in all cases. (b) Individual trial-to-trial glomerulus-odor responses embedded in PC 1-2 space. Responses for the same flies as Figure 1k-1 are shown. Each linked color represents one fly. Trial 1 and trial 2 responses are shown for ORN left lobe (upper left), ORN right lobe (upper right), PN left lobe (lower left), and PN right lobe (lower right). (c) Distance in the full glomerulus-odor response space between recordings within a lobe (trial-to-trial), across lobes (within fly), and across flies for ORNs and PNs. Points represent the median value, boxes represent the interquartile range, and whiskers the range of the data. 
bioRxiv preprint doi: https://doi.org/10.1101/2021.12.24.474127; this version posted December 24, 2021. The copyright holder for this preprint (which was not certified by peer review) is the author/funder, who has granted bioRxiv a license to display the preprint in perpetuity. It is made available under aCC-BY 4.0 International license.

Churgin \& Lavrentovich et al., 2021 - preprint version - www.biorxiv.org
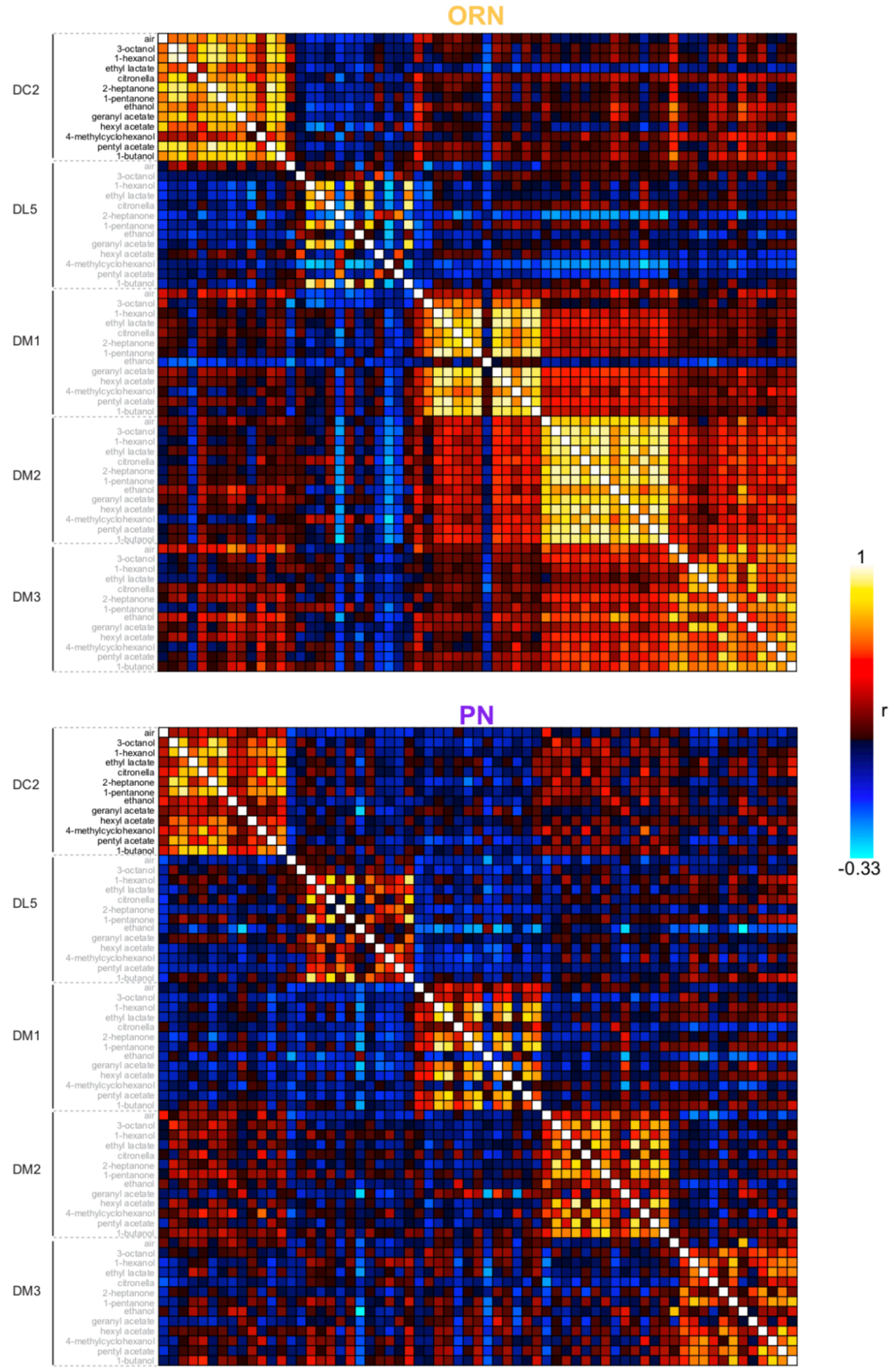

Supplementary Figure 6: Calcium response correlation matrices. Correlation between calcium response dimensions across flies measured in ORNs (top) and PNs (bottom). Glomerulus-odor responses are correlated at the level of glomeruli in both cell types. Inter-glomerulus correlations are more prominent in ORNs than PNs, consistent with known AL transformations that result in decorrelated PN responses ${ }^{18,75}$. 
bioRxiv preprint doi: https://doi.org/10.1101/2021.12.24.474127; this version posted December 24,2021 . The copyright holder for this preprint (which was not certified by peer review) is the author/funder, who has granted bioRxiv a license to display the preprint in perpetuity. It is made available under aCC-BY 4.0 International license.

Churgin \& Lavrentovich et al., 2021 - preprint version - www.biorxiv.org

a ORN PC loadings grouped by glomerulus

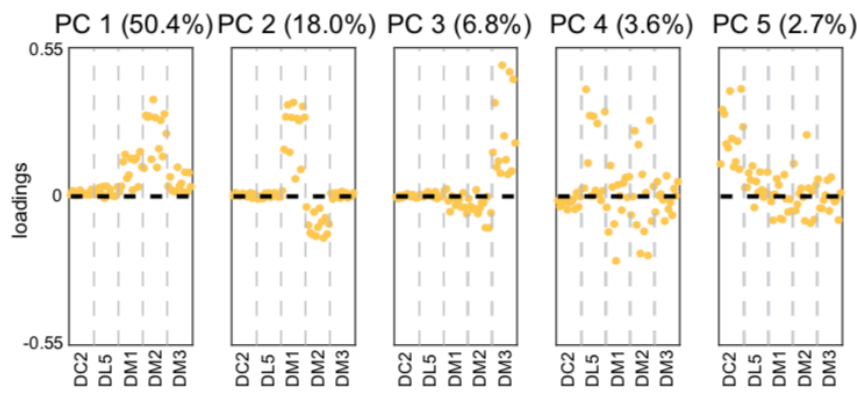

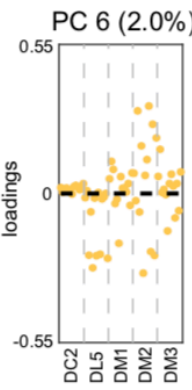

C ORN PC loadings grouped by odor
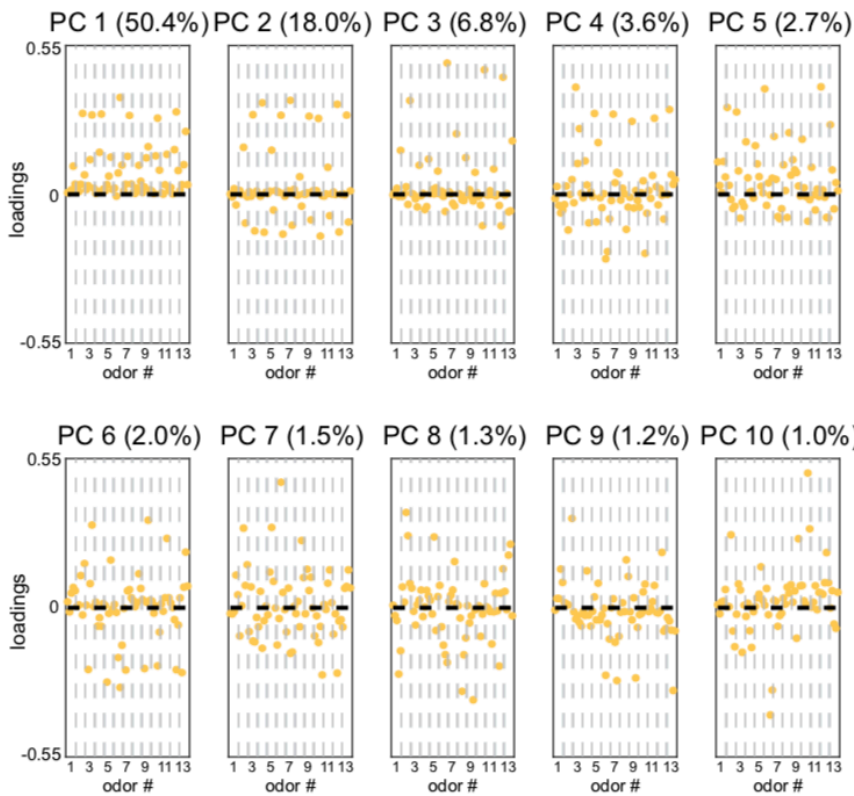

b

PN PC loadings grouped by glomerulus
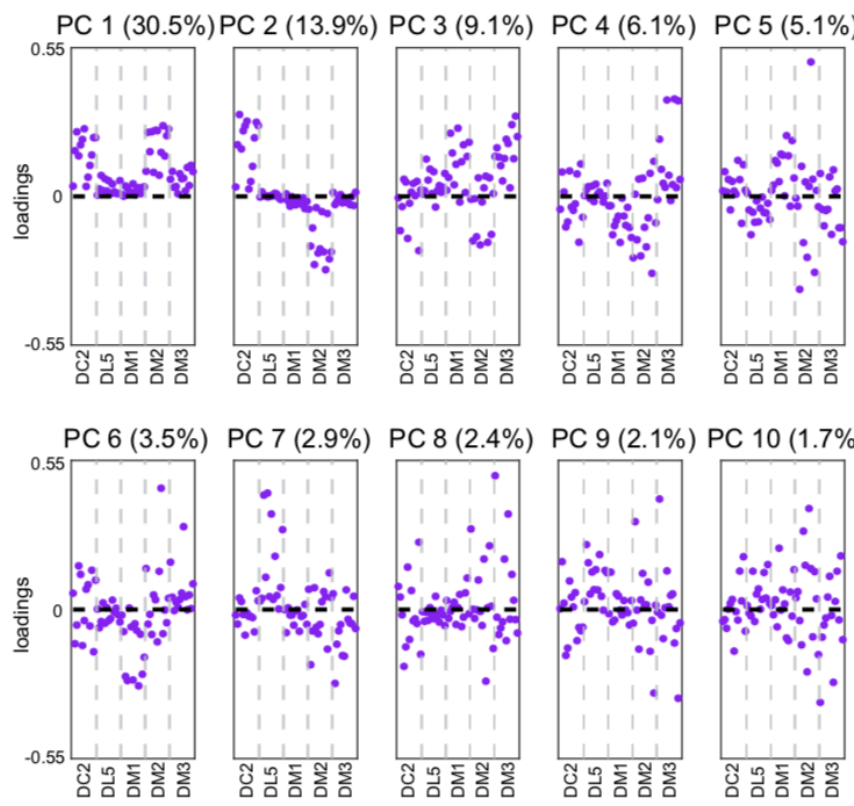

d

PN PC loadings grouped by odor
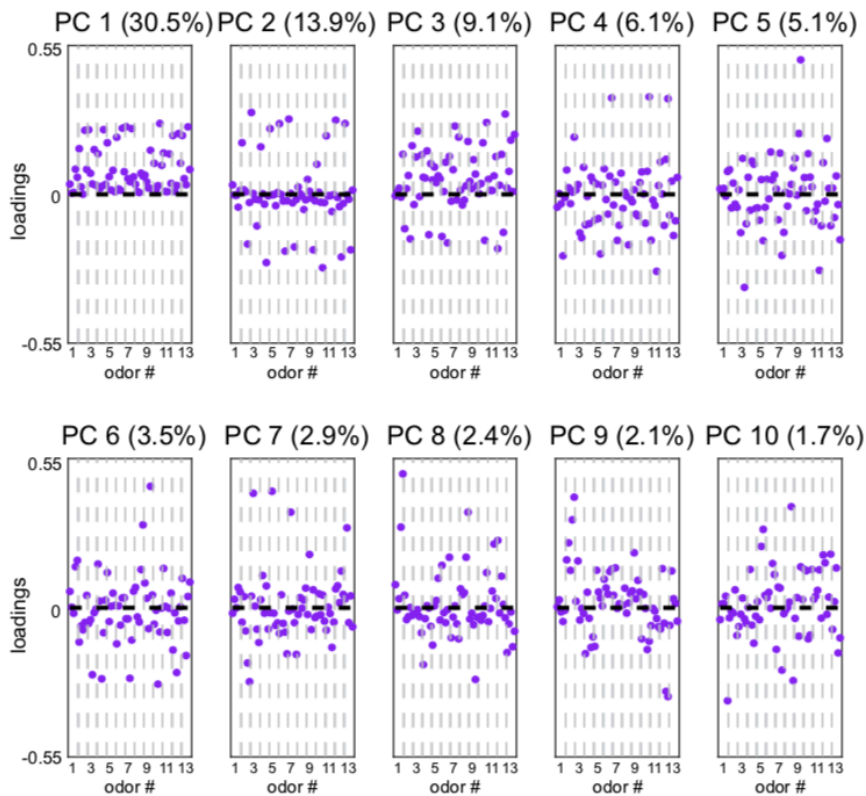

Supplementary Figure 7: Calcium imaging principal component loadings (a-b) First 10 principal component loadings measured from calcium responses in ORNs ( $a, n=65$ flies) and PNs (b, $n=122$ flies). Loadings are grouped by glomerulus, with each loading within a glomerulus representing the response of that glomerulus to one odor in the odor panel. Odors are the same as those listed in Figure 1g. (c-d) The same 10 principal component loadings as those shown in panels (a-b) grouped by odor rather than glomerulus. Glomeruli within each odor block are given in the order of panels (a) and (b). 
bioRxiv preprint doi: https://doi.org/10.1101/2021.12.24.474127; this version posted December 24, 2021. The copyright holder for this preprint (which was not certified by peer review) is the author/funder, who has granted bioRxiv a license to display the preprint in perpetuity. It is made available under aCC-BY 4.0 International license.

Churgin \& Lavrentovich et al., 2021 - preprint version - www.biorxiv.org

a

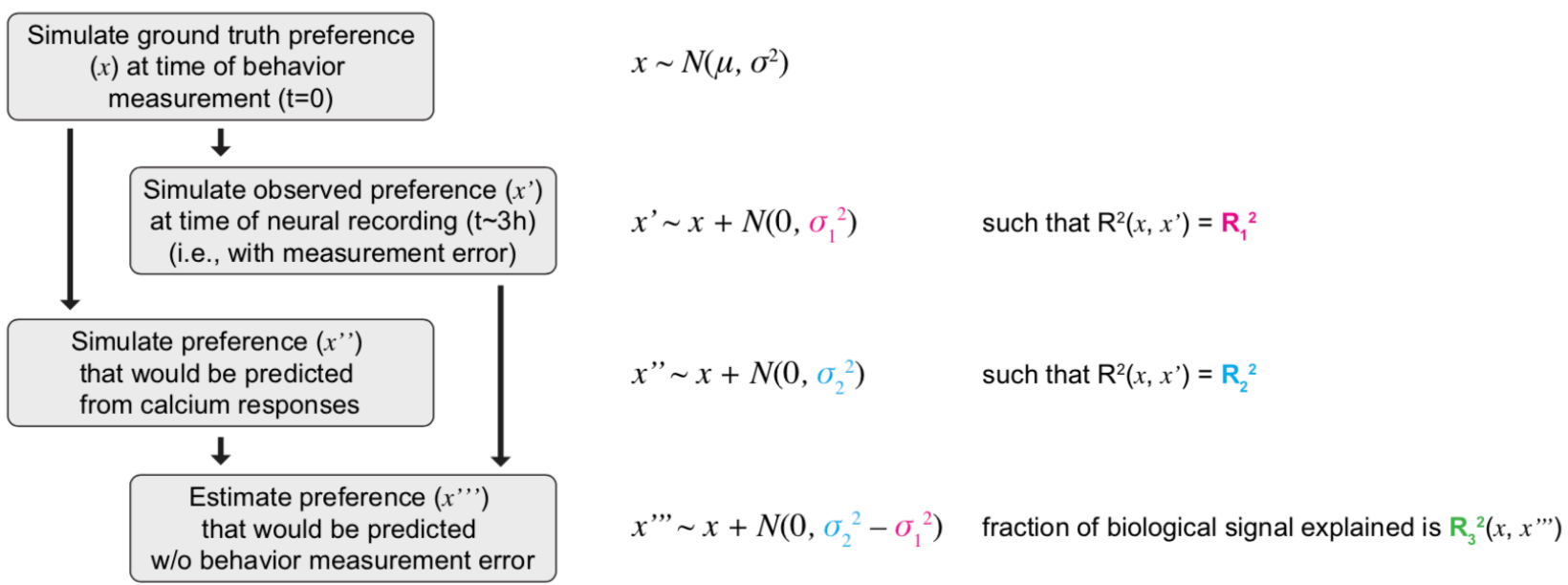

b

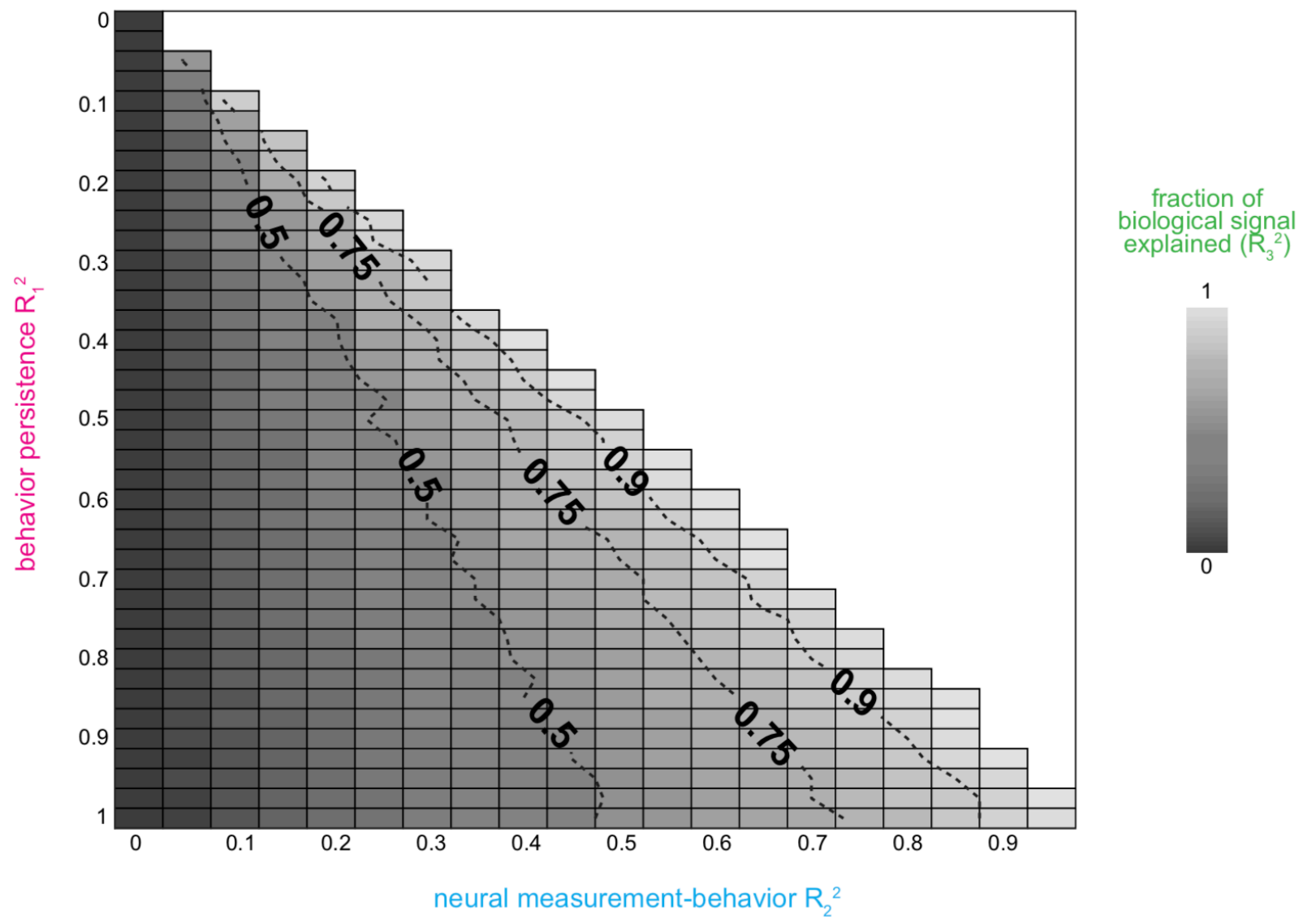

Supplementary Figure 8: Estimating the biological signal explained by Ca-behavior models (a) Schematic of modeling approach to estimate the fraction of biological signal variance ( $\mathrm{R}_{3}{ }^{2}$, green), as opposed to noise variance, explained by calcium response-behavior models. (b) Estimated fraction of behavioral variance explained $\left(\mathrm{R}_{3}{ }^{2}\right.$, green) by calcium response-behavior models (colormap) as a function of the behavioral persistence $\left(\mathrm{R}_{1}^{2}, \mathrm{y}\right.$-axis, pink) and neural-behavior variance explained $\left(\mathrm{R}_{2}^{2}, \mathrm{x}\right.$-axis, blue). $\mathrm{R}_{1}{ }^{2}$ can be estimated from the repeatability of behavior measures over the timescale of the experiment (Fig S1b-e) and $\mathrm{R}_{2}^{2}$ is the calcium response-behavior model performance (e.g., Fig11-n, p-r, Fig3c,f,i). 
bioRxiv preprint doi: https://doi.org/10.1101/2021.12.24.474127; this version posted December 24,2021 . The copyright holder for this preprint (which was not certified by peer review) is the author/funder, who has granted bioRxiv a license to display the preprint in perpetuity. It is made available under aCC-BY 4.0 International license.

Churgin \& Lavrentovich et al., 2021 - preprint version - www.biorxiv.org

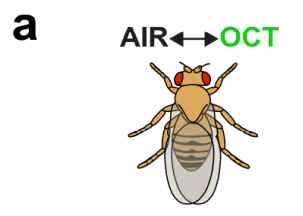

\begin{tabular}{|c|c|c|}
\hline $\begin{array}{c}\text { circuit } \\
\text { element }\end{array}$ & raw $\mathbf{R}^{2}$ & $\begin{array}{c}\text { \% of } \\
\text { behavior } \\
\text { variation }\end{array}$ \\
\hline $\begin{array}{c}\text { ORN } \\
\mathrm{Ca}^{++}\end{array}$ & $0.23-0.25$ & 80 \\
\hline $\begin{array}{c}\text { ORN pre- } \\
\text { synapse } \\
\text { density }\end{array}$ & n.d. & n.d. \\
\hline $\begin{array}{c}\mathrm{PN}^{\mathrm{Ca}} \\
\mathrm{Ca}^{++}\end{array}$ & $0.10-0.16$ & 50 \\
\hline
\end{tabular}
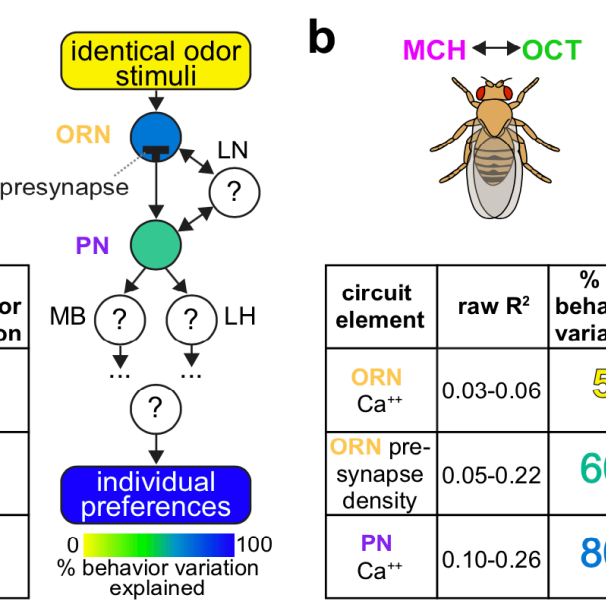

\begin{tabular}{|c|c|c|}
\hline $\begin{array}{c}\text { circuit } \\
\text { element }\end{array}$ & raw $\mathbf{R}^{2}$ & $\begin{array}{c}\text { \% of } \\
\text { behavior } \\
\text { variation }\end{array}$ \\
\hline $\begin{array}{c}\text { ORN } \\
\text { Ca }^{++}\end{array}$ & $0.03-0.06$ & 5 \\
\hline $\begin{array}{c}\text { ORN pre- } \\
\text { synapse } \\
\text { density }\end{array}$ & $0.05-0.22$ & 60 \\
\hline $\begin{array}{c}\text { PN } \\
\mathrm{Ca}^{++}\end{array}$ & $0.10-0.26$ & 80 \\
\hline
\end{tabular}

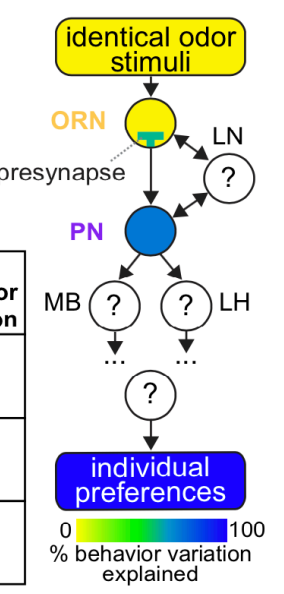

Supplementary Figure 9: Loci of individuality across the olfactory periphery (a) Table summarizing circuit element predictors, the strength of their nominal correlation with odor-vs-air behavior scores, and the estimated fraction of biological signal captured by these models, as determined by the analysis presented in Fig S8. Schematic at right places these values in the context of the olfactory circuit. (b) As in (a) but for odor-vs-odor experiments.
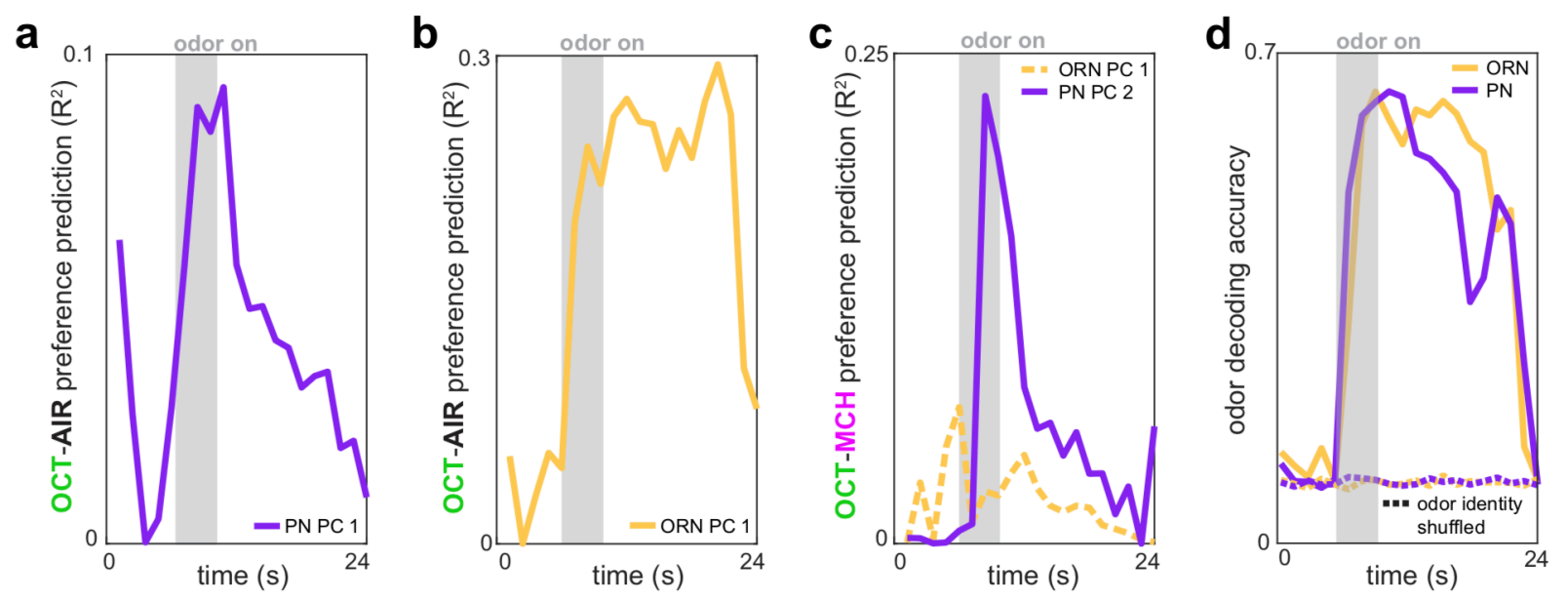

Supplementary Figure 10: Time-dependent preference- and odor-decoding (a) R 2 of odor-vs-air preference predicted by PC 1 of PN activity as a function of time across trials ( $\mathrm{n}=53$ flies). (b) $\mathrm{R}^{2}$ of odor-vs-air preference predicted by PC 1 of ORN activity as a function of time across trials ( $n=30$ flies). (c) $\mathrm{R}^{2}$ of odor-vs-odor preference predicted by PC 2 of PN activity (solid plum, $n=69$ flies) or PC 1 of ORN activity (dashed peach, $\mathrm{n}=35$ flies) as a function of time across trials. (d) Logistic regression classifier accuracy of decoding odor identity from 5 glomerular responses as a function of time. Dashed curves indicate performance on shuffled data. 
bioRxiv preprint doi: https://doi.org/10.1101/2021.12.24.474127; this version posted December 24,2021 . The copyright holder for this preprint (which was not certified by peer review) is the author/funder, who has granted bioRxiv a license to display the preprint in perpetuity. It is made available under aCC-BY 4.0 International license.

Churgin \& Lavrentovich et al., 2021 - preprint version - www.biorxiv.org

a
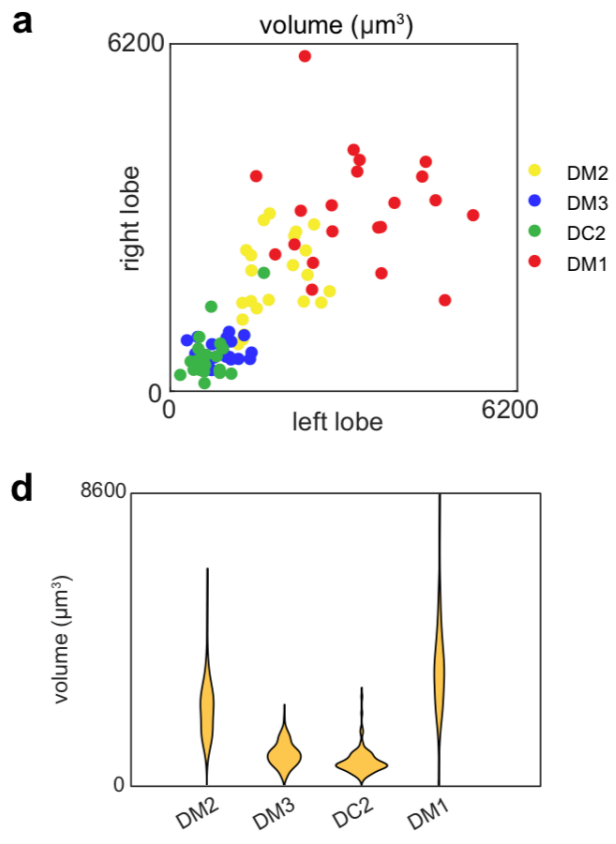

b

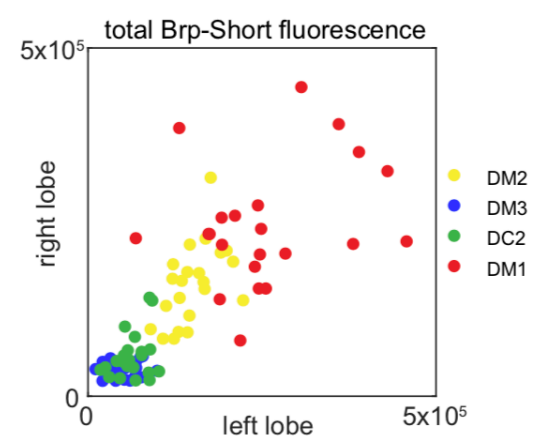

e

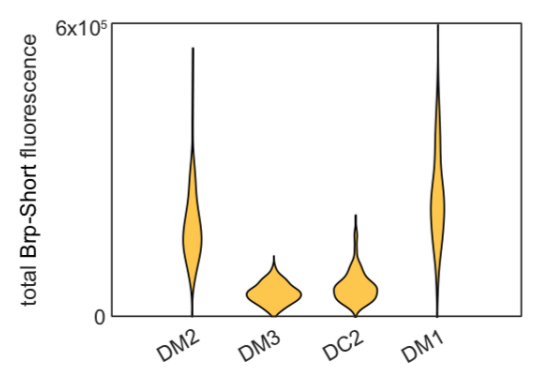

C

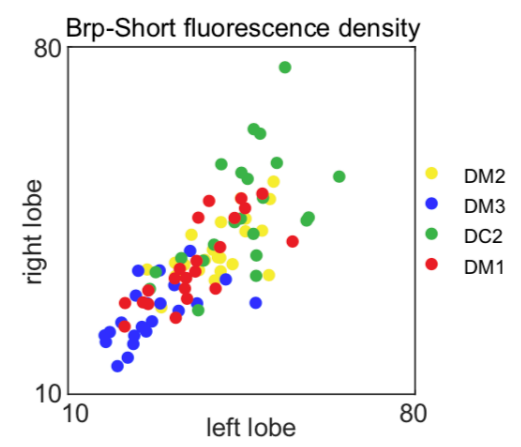

f

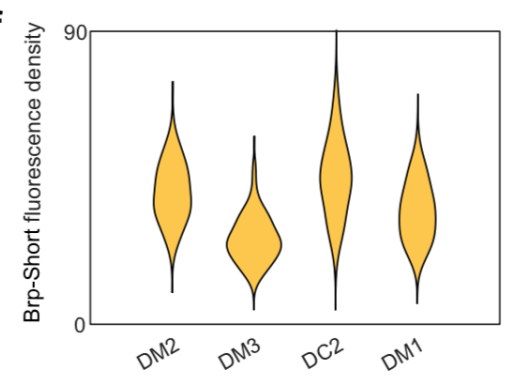

g

training data only

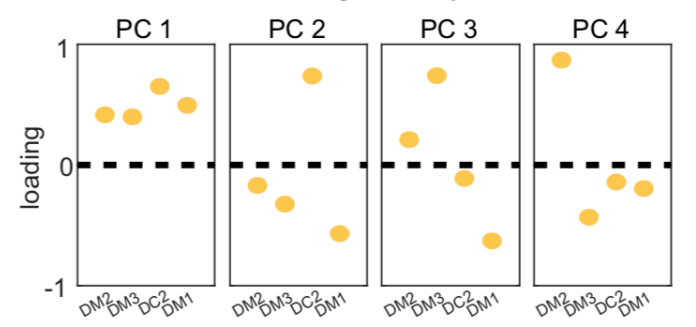

h

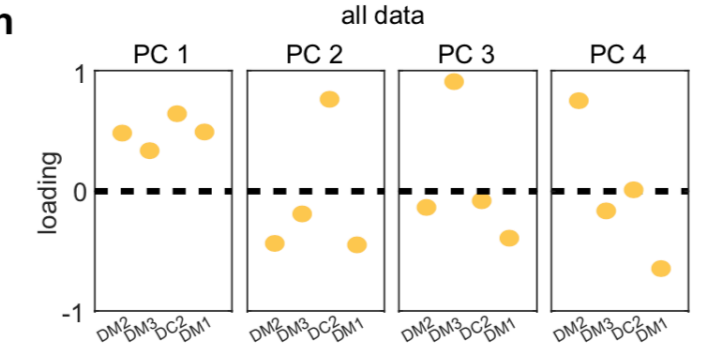

Supplementary Figure 11: ORN>Brp-Short characterization and model predictions (a-c) Right versus left glomerulus properties measured from z-stacks of stained Orco>Brp-Short samples. (a) Volume, (b) total Brp-Short fluorescence, (c) Brp-Short fluorescence density. (d-f) Same data as panels (a-c) represented in violin plots (kernel density estimated). (g) Principal component loadings of Brp-Short density calculated using only training data (n=22 flies). (h) Principal component loadings of Brp-Short density calculated using all data ( $\mathrm{n}=53$ flies). 
bioRxiv preprint doi: https://doi.org/10.1101/2021.12.24.474127; this version posted December 24,2021 . The copyright holder for this preprint (which was not certified by peer review) is the author/funder, who has granted bioRxiv a license to display the preprint in perpetuity. It is made available under aCC-BY 4.0 International license.

Churgin \& Lavrentovich et al., 2021 - preprint version - www.biorxiv.org

a

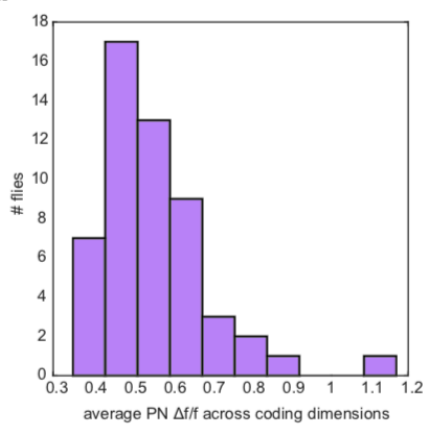

C

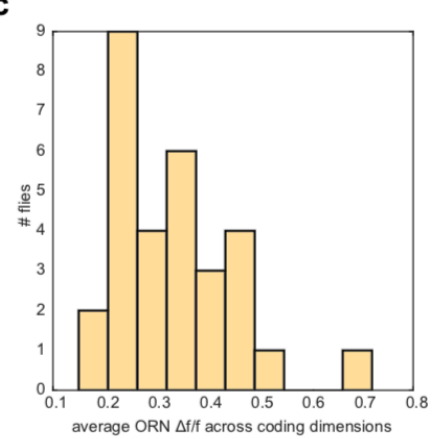

e

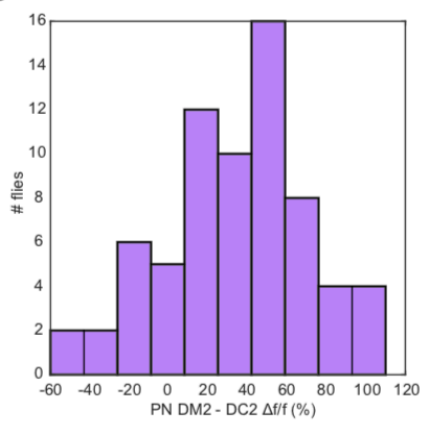

g

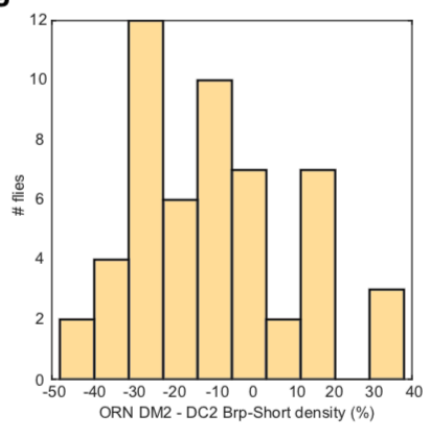

b

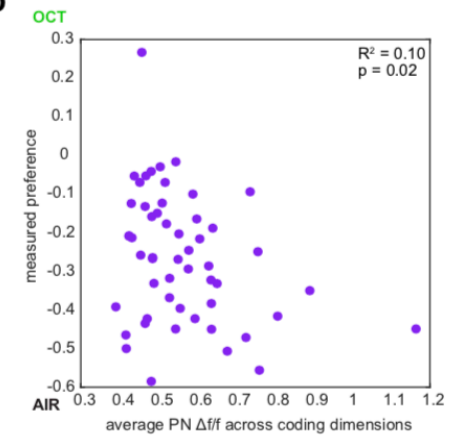

d

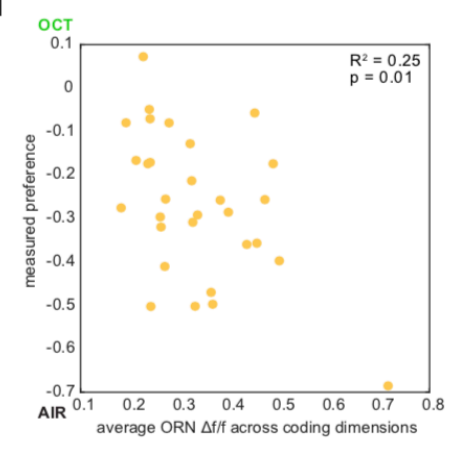

f

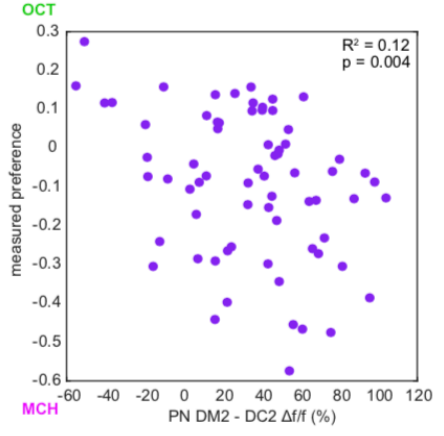

h

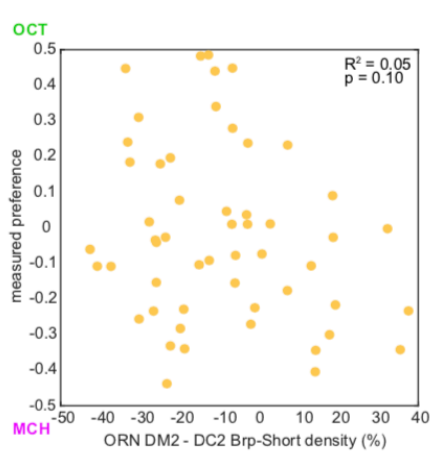

Supplementary Figure 12: Calcium and Brp-Short predictor variation (a) Histogram of average PN $\Delta \mathrm{f} / \mathrm{f}$ across all coding dimensions in flies in which OCT-AIR preference was measured. (b) Measured OCT-AIR preference versus average PN $\Delta \mathrm{f} / \mathrm{f}$ ( $\mathrm{n}=53 \mathrm{flies}$ ). (c) Histogram of average ORN $\Delta \mathrm{f} / \mathrm{f}$ across all coding dimensions in flies in which OCT-AIR preference was measured. (d) Measured OCT-AIR preference versus average ORN $\Delta \mathrm{f} / \mathrm{f}$ $(\mathrm{n}=30$ flies). (e) Histogram of $\% \Delta \mathrm{f} / \mathrm{f}$ difference between DM2 and DC2 PN responses in flies in which OCT-MCH preference was measured. (f) Measured OCT-MCH preference versus \% $\Delta \mathrm{f} / \mathrm{f}$ difference between DM2 and DC2 PN responses df/f ( $\mathrm{n}=69 \mathrm{flies}$ ). (g) Histogram of \% Brp-Short density difference between DM2 and DC2 ORNs in flies in which OCT-MCH preference was measured. (h) Measured OCT-MCH preference versus $\%$ Brp-Short density difference between DM2 and DC2 ORNs ( $=53$ flies). 
bioRxiv preprint doi: https://doi.org/10.1101/2021.12.24.474127; this version posted December 24, 2021. The copyright holder for this preprint (which was not certified by peer review) is the author/funder, who has granted bioRxiv a license to display the preprint in perpetuity. It is made available under aCC-BY 4.0 International license.

Churgin \& Lavrentovich et al., 2021 - preprint version - www.biorxiv.org
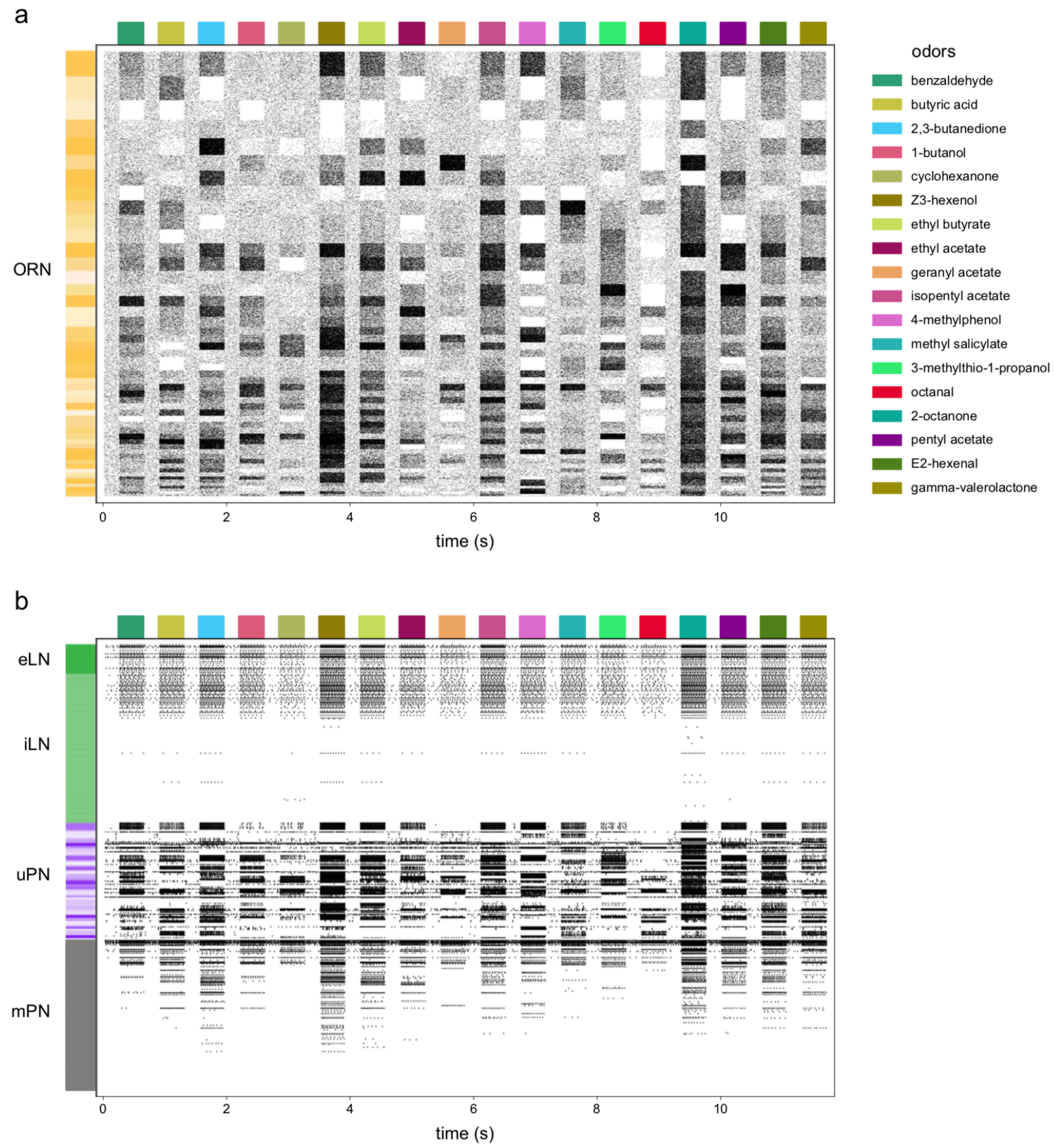

Supplementary Figure 13: AL model raster plot (a) Action potential raster plot of ORNs in the baseline simulated AL. Rows are individual ORNs, black ticks indicate action potentials. Random shades of orange at left indicate blocks of ORN rows projecting to the same glomerulus. (b) As in (a) for the remaining neurons in the model. Shades of green indicate excitatory vs inhibitory LNs and shades of purple indicate PNs with dendrites in the same glomeruli. 
bioRxiv preprint doi: https://doi.org/10.1101/2021.12.24.474127; this version posted December 24,2021 . The copyright holder for this preprint (which was not certified by peer review) is the author/funder, who has granted bioRxiv a license to display the preprint in perpetuity. It is made available under aCC-BY 4.0 International license.

Churgin \& Lavrentovich et al., 2021 - preprint version - www.biorxiv.org
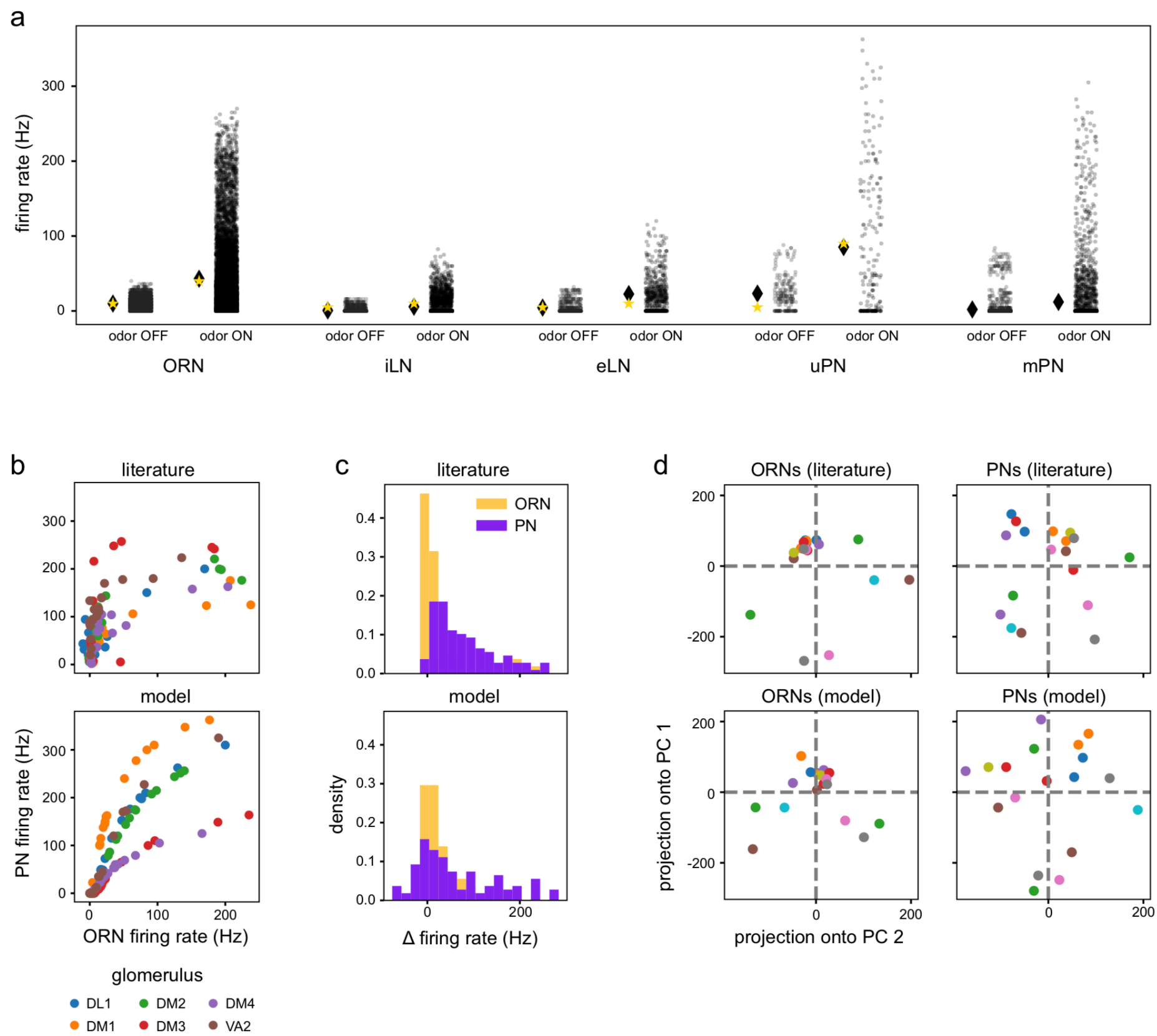
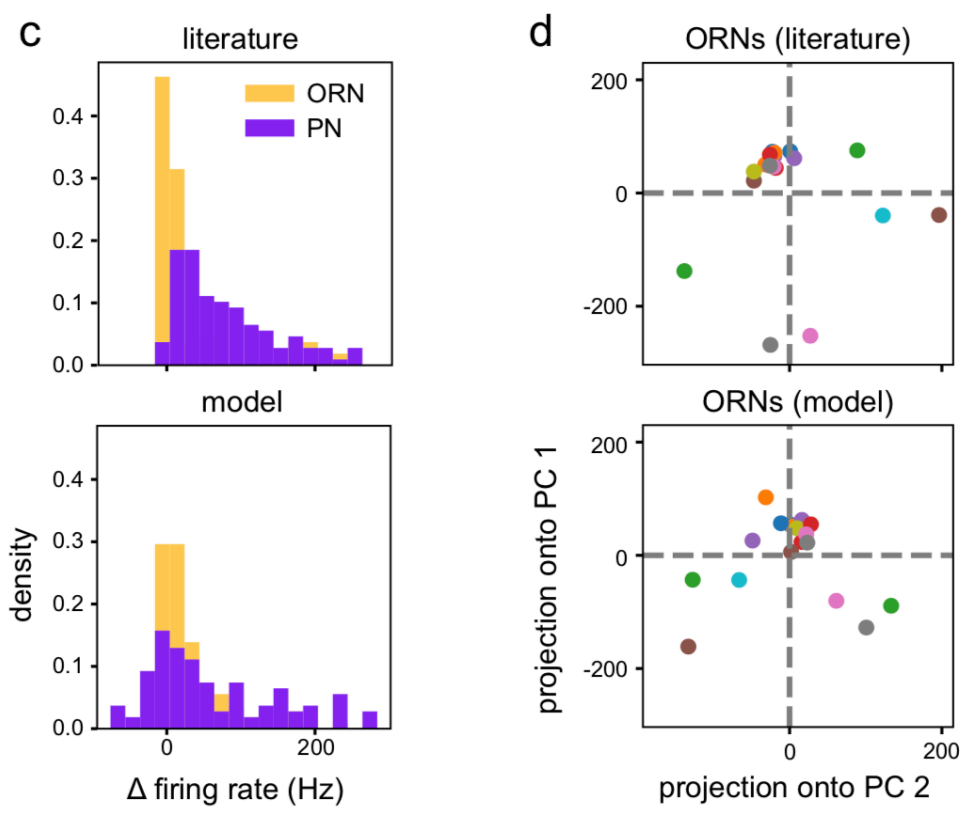

PNs (literature)

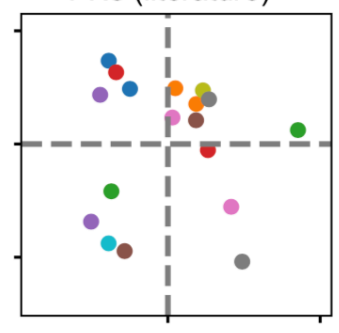

PNs (model)

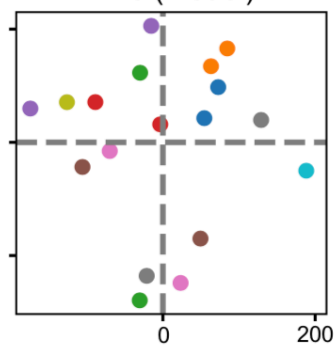

Supplementary Figure 14: AL model baseline outputs compared to experimental data (a) Distributions of model neuron firing rates by cell type across odors (transparent black points are individual neuron-odor combinations). Black lozenge symbols indicate the mean firing rate of the points to the right. Yellow stars indicate the comparable experimental values reported in 15,21,45,68. (b) Scatter plots of average PN firing rate vs ORN firing rate during odor stimuli in the model vs experimental values ${ }^{18}$. Points are odors, colors are glomeruli. (c) Histograms of ON odor minus OFF odor glomerulus-average PN and ORN firing rates in the model vs experimental values ${ }^{18}$, showing flatter distributions in PNs. (d) Odor representations in the first 2 PCs of glomerulus-average ORN responses and PN responses in the model and experimental results ${ }^{18}$. Points are odors. Pairwise distances between PN representations are more uniform than in ORNs in both the model and experimental data. Panels (b)-(d) use glomerulus-average PN and ORN firing rates from six of the seven glomeruli in ${ }^{18}$, as VM2 is significantly truncated in the hemibrain ${ }^{34}$. Literature features in panels (b)-(d) were extracted from ${ }^{18}$ using WebPlotDigitizer ${ }^{76}$. 
bioRxiv preprint doi: https://doi.org/10.1101/2021.12.24.474127; this version posted December 24,2021 . The copyright holder for this preprint (which was not certified by peer review) is the author/funder, who has granted bioRxiv a license to display the preprint in perpetuity. It is made available under aCC-BY 4.0 International license.

Churgin \& Lavrentovich et al., 2021 - preprint version - www.biorxiv.org
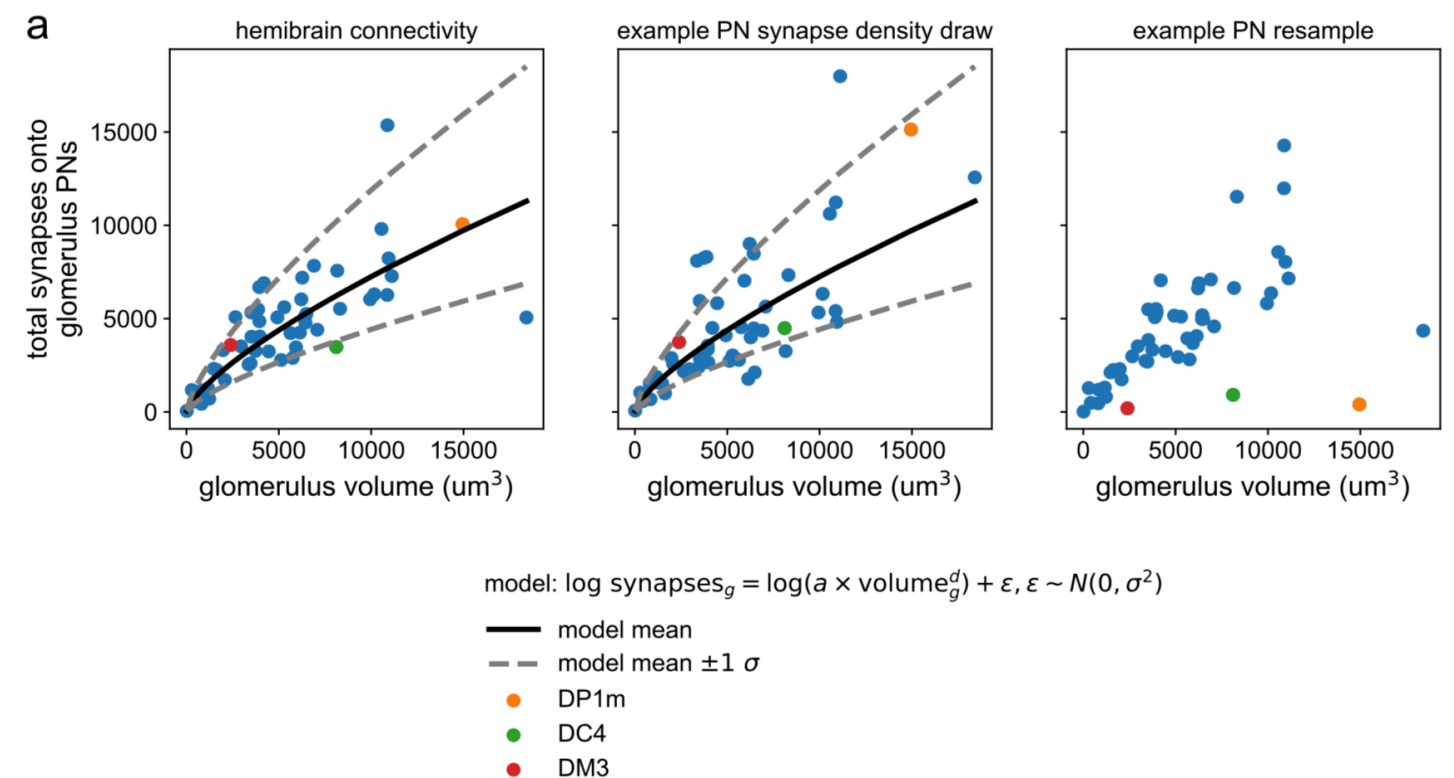

b

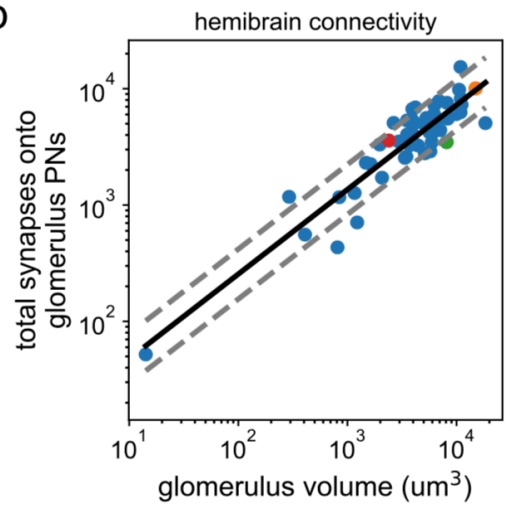

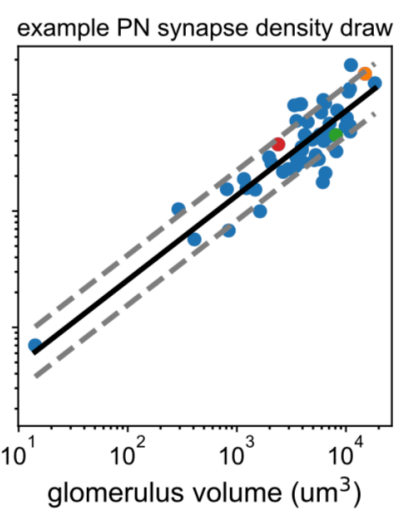

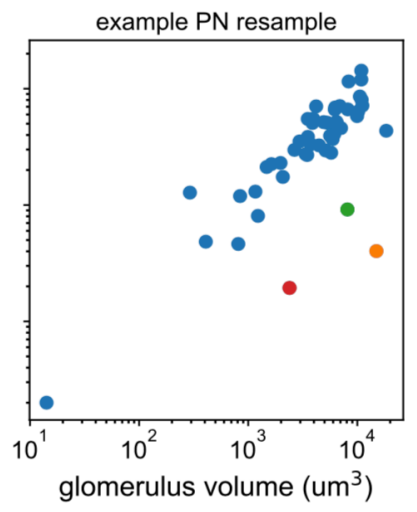

Supplementary Figure 15: Synapse counts vs glomerular volume in the hemibrain and AL model (a) Left) Scatter plot of total PN input synapses within a glomerulus vs that glomerulus' volume from the hemibrain data set. Solid line represents the maximum likelihood-fit mean synapse count vs glomerular volume, and dashed lines the fit $+/-1$ standard deviation. Middle) As (left) but for a single sample from the parameterized distribution of PN input synapses vs glomerular volume. Right) As in previous for a single bootstrap resample of PNs. Colorhighlighted glomeruli illustrate that when PNs within a glomerulus have highly asymmetrical synapse counts, bootstrapping them alone can result in apparent synapse densities that lie outside the empirical distribution (left). (b) As in (a) but on log-log axes, showing the linear relationship between synapse density and glomerular volume after this transformation, and bootstrapped densities falling outside this distribution at right. 
bioRxiv preprint doi: https://doi.org/10.1101/2021.12.24.474127; this version posted December 24,2021 . The copyright holder for this preprint (which was not certified by peer review) is the author/funder, who has granted bioRxiv a license to display the preprint in perpetuity. It is made available under aCC-BY 4.0 International license.

a

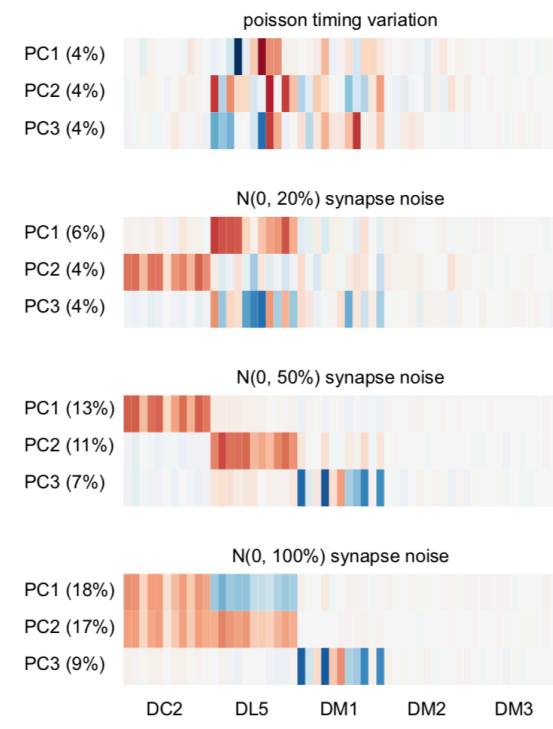

b
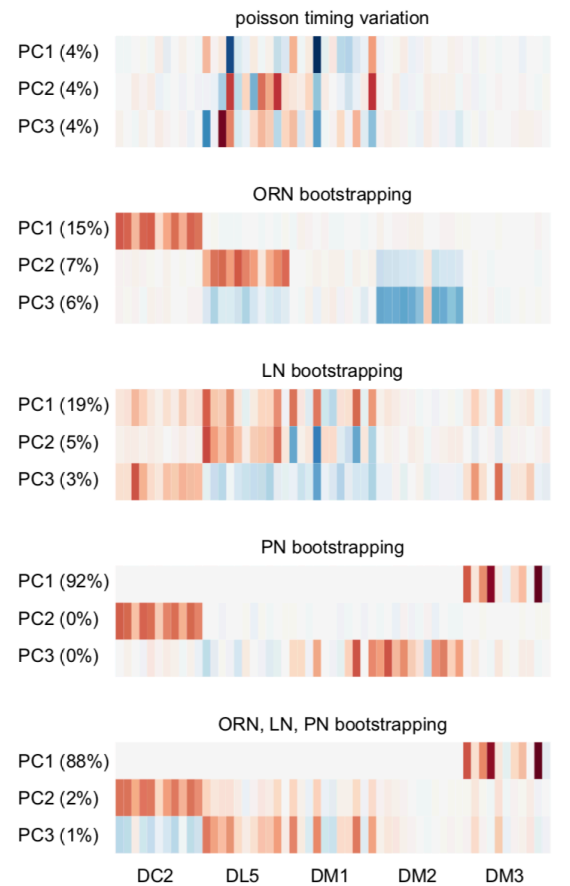

C
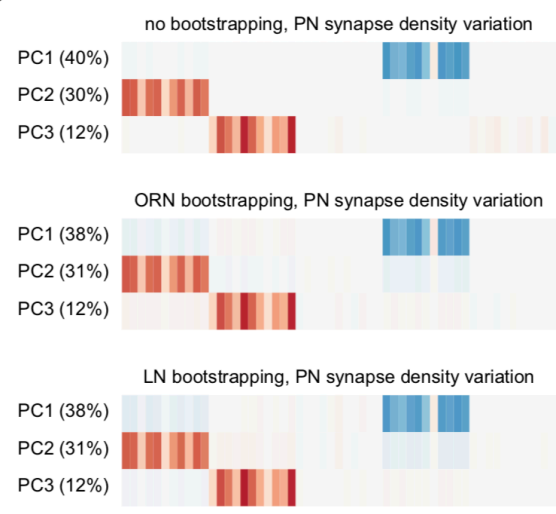

PN bootstrapping, PN synapse density variation PC1 $(31 \%)$ PC3 $(16 \%)$

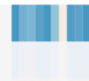

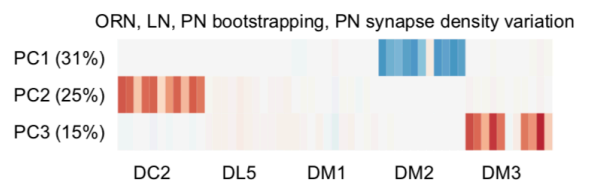

Supplementary Figure 16: PN response PCA loadings under various sources of circuit idiosyncrasy (a) Loadings of the principal components of PN glomerulus-odor responses as simulated across AL models where Gaussian noise with a standard deviation equal to 0, 20, 50, and 100\% of each synapse weight was added to each synaptic weight in the hemibrain data set. (b) As in (a) but with circuit variation coming from bootstrapping of each major AL cell type or all three simultaneously. (c) As in other panels, but with circuit variation coming from bootstrap resampling of different cell-type combinations in addition to PN input synapse density resampling as illustrated in Figure S15. 
bioRxiv preprint doi: https://doi.org/10.1101/2021.12.24.474127; this version posted December 24, 2021. The copyright holder for this preprint (which was not certified by peer review) is the author/funder, who has granted bioRxiv a license to display the preprint in perpetuity. It is made available under aCC-BY 4.0 International license.

Churgin \& Lavrentovich et al., 2021 - preprint version - www.biorxiv.org
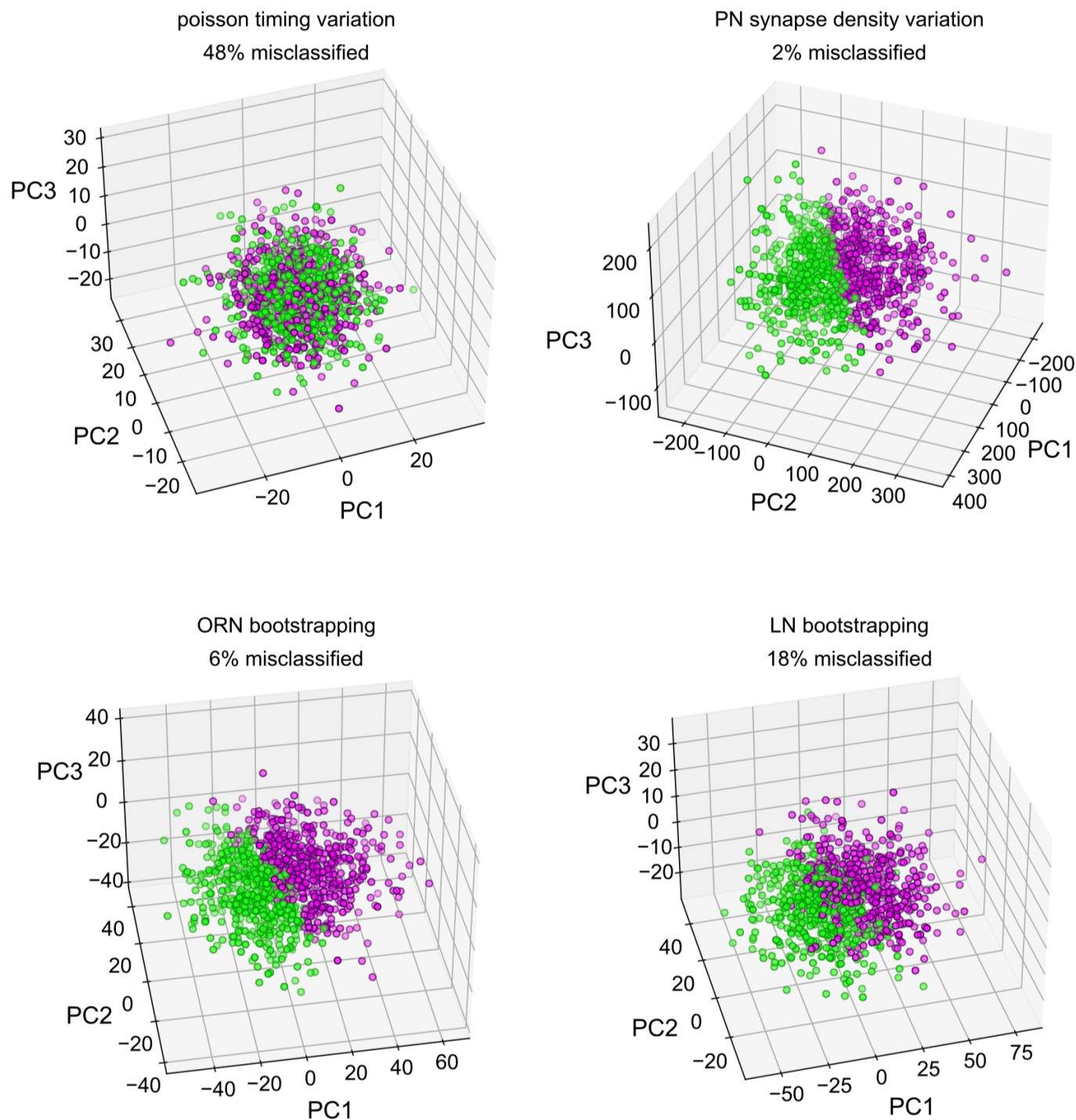

Supplementary Figure 17: Classifiability of simulated idiosyncratic behavior under different sources of circuit idiosyncrasy. Simulated PN odor-glomerulus firing rates projected into their first 3 principal components. Individual points represent single runs of resampled AL models, under four different sources of idiosyncratic variation. PN responses in all odor-glomerulus dimensions were used to calculate simulated behavior scores for each resampled AL, by applying the PN calcium-odor-vs-odor linear model (Fig $2 \mathrm{~g}$ ). Magenta points represent flies with simulated preference for $\mathrm{MCH}$ in the top 50\%, and green OCT preference. \% Misclassification refers to $100 \%$ - the accuracy of a linear classifier trained on MCH-vsOCT preference in the space of the first three PCs. This measures how much of the variance along the PN calcium-odor-vs-odor linear model lies outside the first three PCs of simulated PN variation. 
bioRxiv preprint doi: https://doi.org/10.1101/2021.12.24.474127; this version posted December 24, 2021. The copyright holder for this preprint (which was not certified by peer review) is the author/funder, who has granted bioRxiv a license to display the preprint in perpetuity. It is made available under ACC-BY 4.0 International license.

Churgin \& Lavrentovich et al., 2021 - preprint version - www.biorxiv.org

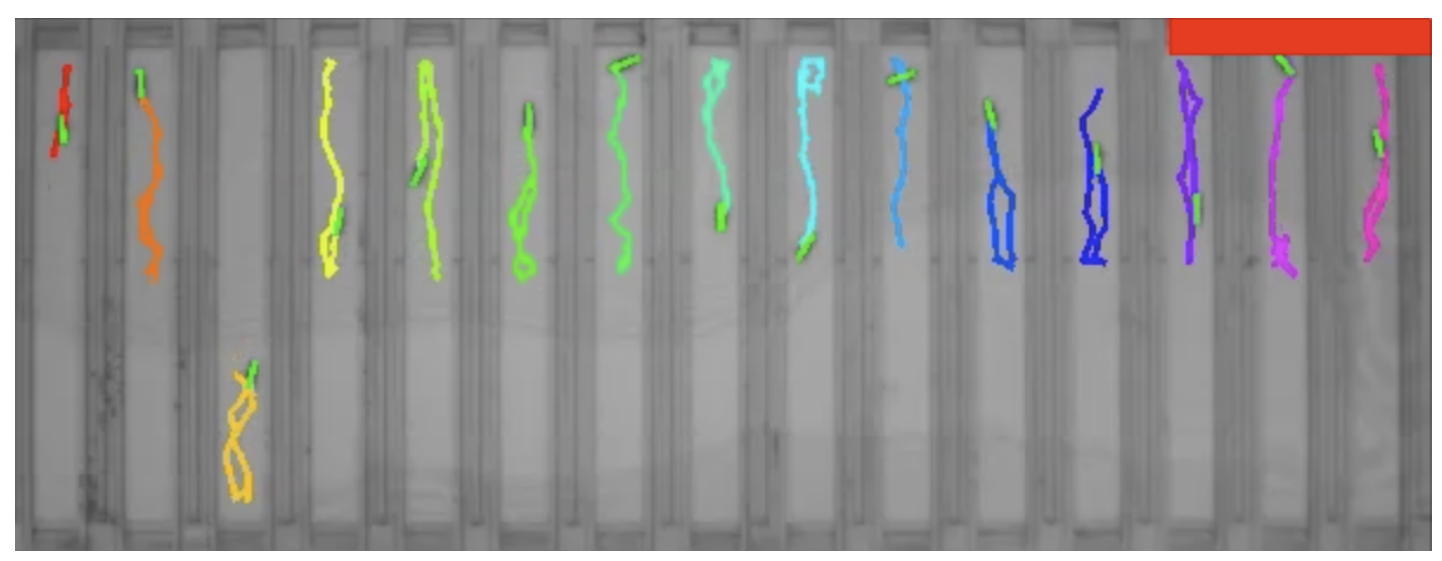

Movie 1: Example recording with automated tracking of an odor-vs-air behavioral assay. The recent positions of each fly (green line) are shown in different colors. Red bar indicates when the odor stream is turned on.

http://lab.debivort.org/odor-loci-of-individuality/movies/OLOI-Movie-1-odor-vs-air-behavior.mp 4

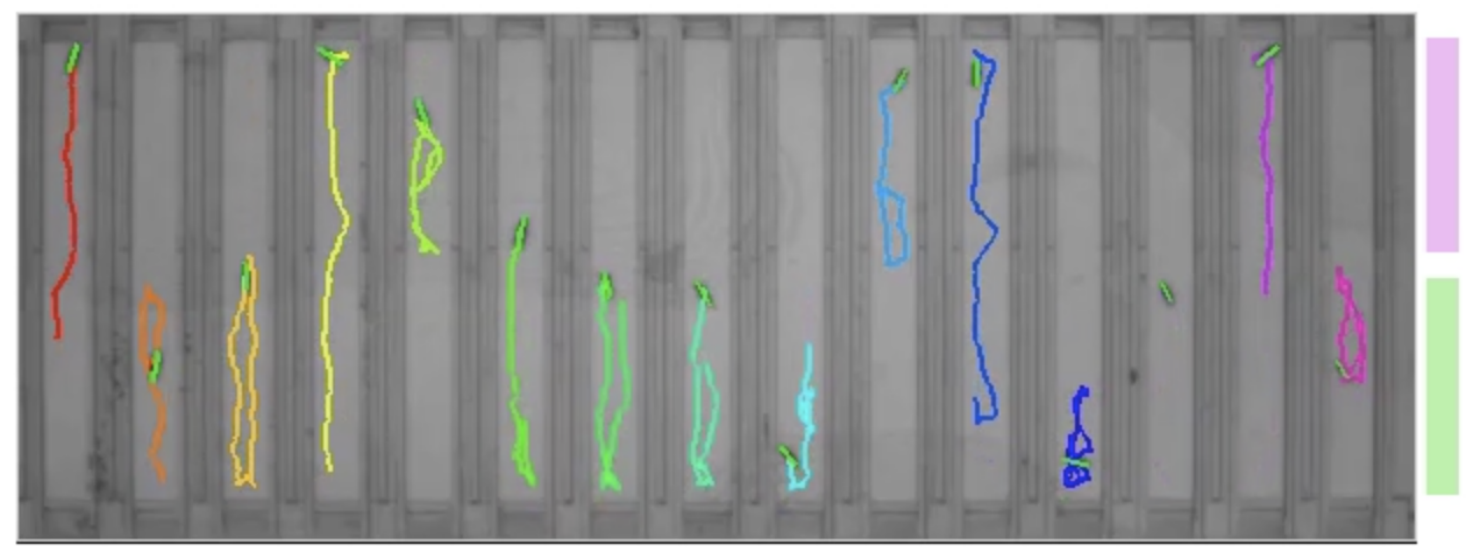

Movie 2: Example recording with automated tracking of an odor-vs-odor behavioral assay. The recent positions of each fly (green line) are shown in different colors. Magenta and green bars at right indicate when MCH and OCT are respectively flowing into the top and bottom halves of each arena. http://lab.debivort.org/odor-loci-of-individuality/movies/OLOI-Movie-2-odor-vs-odor-behavior.mp4 
bioRxiv preprint doi: https://doi.org/10.1101/2021.12.24.474127; this version posted December 24, 2021. The copyright holder for this preprint (which was not certified by peer review) is the author/funder, who has granted bioRxiv a license to display the preprint in perpetuity. It is made available under aCC-BY 4.0 International license.

Churgin \& Lavrentovich et al., 2021 - preprint version - www.biorxiv.org

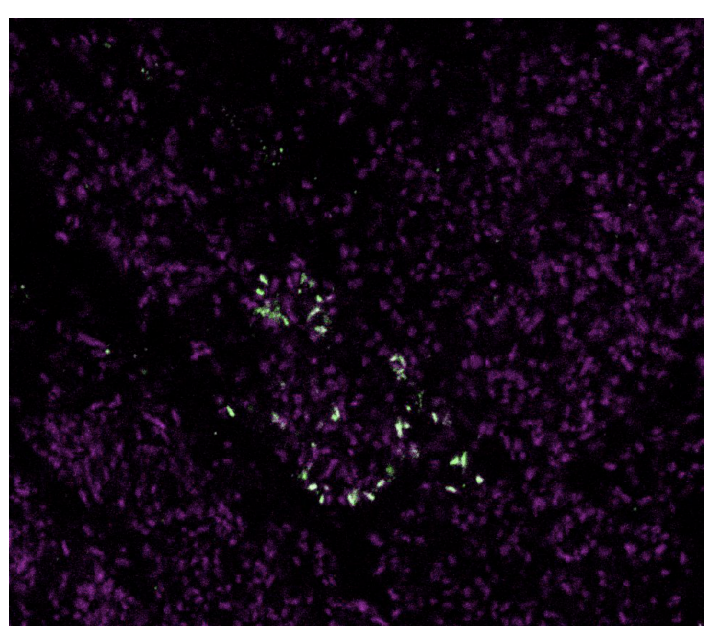

Movie 3: Confocal image stack of expanded DC2>Brp-Short. Magenta is nc82 stain, Green is Or13a>Brp-Short. Frames are z-slices spaced at $0.54 \mu \mathrm{m}$. Image height corresponds to a post-expansion field of view of 107 x $90 \mu \mathrm{m}(\mathrm{a} \sim 2.5 \mathrm{x}$ linear expansion factor $)$. http://lab.debivort.org/odorloci-of-individuality/movies/OLOI-Movie-3-Brp-expansion-stack.avi

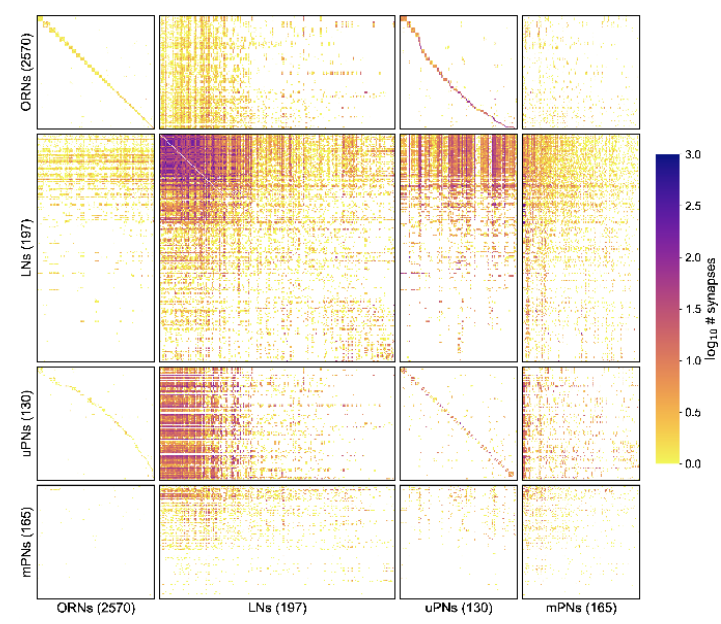

Movie 4: Simulated AL connectivity matrices under glomerular density resampling. Each frame corresponds to the hemibrain connectome synaptic weights, rescaled according to a sample from the relationship between synapse count and volume parameterized in Fig S15.

http://lab.debivort.org/odor-loci-of-individuality/movies/OLOI-Movie-4-synapse-density-resampling.gif 
bioRxiv preprint doi: https://doi.org/10.1101/2021.12.24.474127; this version posted December 24, 2021. The copyright holder for this preprint (which was not certified by peer review) is the author/funder, who has granted bioRxiv a license to display the preprint in perpetuity. It is made available under aCC-BY 4.0 International license.

Churgin \& Lavrentovich et al., 2021 - preprint version - www.biorxiv.org

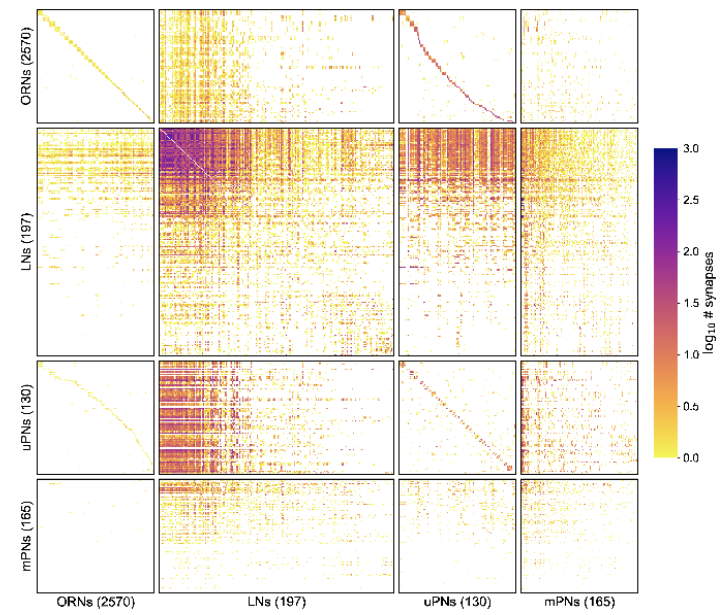

Movie 5: Simulated AL connectivity matrices under ORN bootstrapping. Each frame corresponds to the hemibrain connectome synaptic weights, but with the population of ORNs projecting to each glomerulus resampled with replacement. See Modeling Methods. http:// lab.debivort.org/odor-loci-of-individuality/movies/OLOI-Movie-5-ORN-bootstrapping.gif

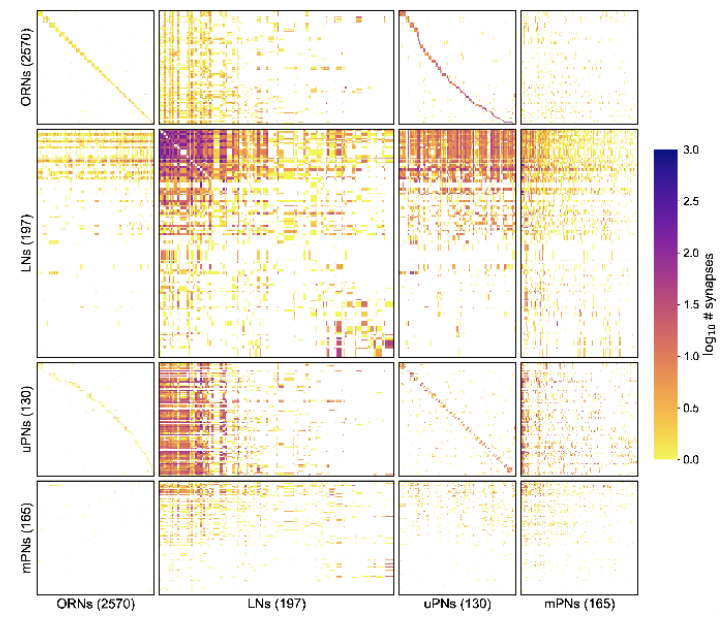

Movie 6: Simulated AL connectivity matrices under LN bootstrapping. Each frame corresponds to the hemibrain connectome synaptic weights, but with the population of LNs resampled with replacement. See Modeling Methods.

http://lab.debivort.org/odor-loci-of-individuality/movies/OLOI-Movie-6-LN-bootstrapping.gif 
bioRxiv preprint doi: https://doi.org/10.1101/2021.12.24.474127; this version posted December $24,2021$. The copyright holder for this preprint (which was not certified by peer review) is the author/funder, who has granted bioRxiv a license to display the preprint in perpetuity. It is made available under aCC-BY 4.0 International license.

Churgin \& Lavrentovich et al., 2021 - preprint version - www.biorxiv.org

Table 1: Calcium response-behavior model statistics

\begin{tabular}{|c|cccccc|c|}
\hline Behavior measured & Neural Predictor & $\begin{array}{c}\text { Figure } \\
\text { Panel }\end{array}$ & $\mathbf{n}$ & $\boldsymbol{\beta 0}$ & $\boldsymbol{\beta 1}$ & R2 & p-value \\
\hline OCT vs. AIR & PN PC 1 (Fig 2a) & 11 & 18 & -0.258 & -0.079 & 0.161 \\
OCT vs. AIR & PN Average all dimensions & $2 \mathrm{c}$ & 53 & -0.051 & -0.379 & 0.098 & 0.099 \\
OCT vs. AIR & ORN PC 1 (Fig 2d) & $1 \mathrm{n}$ & 30 & -0.286 & -0.053 & 0.230 & 0.022 \\
OCT vs. AIR & ORN Average all dimensions & $2 \mathrm{f}$ & 30 & -0.032 & -0.709 & 0.246 & 0.007 \\
OCT vs. MCH & PN PC 2 (Fig 2g) & $1 \mathrm{p}$ & 47 & -0.058 & -0.081 & 0.154 & 0.006 \\
OCT vs. MCH & PN DM2 - DC2 (\% difference) & $2 \mathrm{i}$ & 69 & -0.032 & -0.004 & 0.120 & 0.004 \\
OCT vs. MCH & ORN PC 1 & $1 \mathrm{r}$ & 35 & -0.138 & -0.027 & -0.031 & 0.315 \\
\hline
\end{tabular}

Table 2: Brp-Short-behavior model statistics

\begin{tabular}{|c|c|c|c|c|c|c|c|}
\hline Behavior measured & Neural Predictor & Figure Panel & $\mathbf{n}$ & $\beta 0$ & $\beta 1$ & $\mathbf{R 2}$ & p-value \\
\hline OCT vs. MCH & ORN PC 2 (train data only) (Fig 3e) & $3 \mathrm{f}$ & 22 & -0.087 & 0.017 & 0.218 & 0.028 \\
\hline OCT vs. MCH & ORN PC 2 (all data) (Extended Figure 9h) & Extended Figure 9j & 53 & -0.019 & 0.012 & 0.088 & 0.031 \\
\hline OCT vs. $\mathrm{MCH}$ & ORN DM2 - DC2 & $3 \mathrm{~h}$ & 53 & -0.051 & -0.007 & 0.053 & 0.096 \\
\hline OCT vs. $\mathrm{MCH}$ & ORN DC2 & Extended Figure 9k & 53 & -0.202 & 0.004 & 0.030 & 0.213 \\
\hline OCT vs. MCH & ORN DM2 & Extended Figure 91 & 53 & -0.014 & 0 & 0 & 0.978 \\
\hline
\end{tabular}

Table 3: Typical electrophysiology features of AL cell types, used as model parameters

\begin{tabular}{|c|c|c|c|}
\hline Parameter & ORNs & LNs & PNs \\
\hline Membrane resting potential & $-70 \mathrm{mV} 38$ & $-50 \mathrm{mV} 39$ & $-55 \mathrm{mV} 40$ \\
\hline Action potential threshold & $-50 \mathrm{mV} 38$ & $-40 \mathrm{mV} 39$ & $-40 \mathrm{mV} 40$ \\
\hline Action potential minimum & $-70 \mathrm{mV} 77$ & $-60 m V 39$ & $-55 \mathrm{mV} 40$ \\
\hline Action potential maximum & $0 \mathrm{mV} 38$ & $0 \mathrm{mV} 39$ & $-30 \mathrm{mV} 78$ \\
\hline Action potential duration & $2 \mathrm{~ms} 40$ & $4 \mathrm{~ms} 39$ & $2 \mathrm{~ms} 40$ \\
\hline Membrane capacitance & $73 \mathrm{pF}$ (assumed $=\mathrm{PNs})$ & $64 \mathrm{pF} 79$ & $73 \mathrm{pF} 79$ \\
\hline Membrane resistance & $1.8 \mathrm{GOhm} 38$ & $1 \mathrm{GOhm} 39$ & $0.3 \mathrm{GOhm}{ }^{40}$ \\
\hline
\end{tabular}

